\title{
The brain speaks : functional and structural neural correlates of language production impairments in classic galactosemia
}

Citation for published version (APA):

Timmers, I. (2014). The brain speaks : functional and structural neural correlates of language production impairments in classic galactosemia. [Doctoral Thesis, Maastricht University]. Maastricht University. https://doi.org/10.26481/dis.20140425it

Document status and date:

Published: 01/01/2014

DOI:

10.26481/dis.20140425it

Document Version:

Publisher's PDF, also known as Version of record

Please check the document version of this publication:

- A submitted manuscript is the version of the article upon submission and before peer-review. There can be important differences between the submitted version and the official published version of record.

People interested in the research are advised to contact the author for the final version of the publication, or visit the DOI to the publisher's website.

- The final author version and the galley proof are versions of the publication after peer review.

- The final published version features the final layout of the paper including the volume, issue and page numbers.

Link to publication

\footnotetext{
General rights rights.

- You may freely distribute the URL identifying the publication in the public portal. please follow below link for the End User Agreement:

www.umlib.nl/taverne-license

Take down policy

If you believe that this document breaches copyright please contact us at:

repository@maastrichtuniversity.nl

providing details and we will investigate your claim.
}

Copyright and moral rights for the publications made accessible in the public portal are retained by the authors and/or other copyright owners and it is a condition of accessing publications that users recognise and abide by the legal requirements associated with these

- Users may download and print one copy of any publication from the public portal for the purpose of private study or research.

- You may not further distribute the material or use it for any profit-making activity or commercial gain

If the publication is distributed under the terms of Article 25fa of the Dutch Copyright Act, indicated by the "Taverne" license above, 


\section{The Brain Speaks: \\ Functional and structural neural correlates of language production impairments in classic galactosemia}

Inge Timmers 
C copyright Inge Timmers, Maastricht 2014

Cover illustration: Kevin van Klaveren @ Tattoo-Dice Cover design: Inge Timmers

Lay-out: Inge Timmers

Production: Gildeprint Drukkerijen

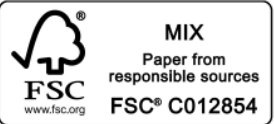

ISBN/EAN 978-94-6108-648-8

The work presented in this thesis is the result of a bi-faculty project of the Faculty of Psychology and Neuroscience and the Faculty of Health, Medicine and Life Sciences of Maastricht University.

The production of this thesis was kindly supported by Stichting Bevordering Kindergeneeskunde, and Galactosemie Vereniging Nederland (GVN). 


\title{
The Brain Speaks: \\ Functional and structural neural correlates of language production impairments in classic galactosemia
}

\author{
DISSERTATION \\ to obtain the degree of Doctor at Maastricht University, \\ on the authority of the Rector Magnificus, Prof. Dr. L.L.G. Soete \\ in accordance with the decision of the Board of Deans, \\ to be defended in public \\ on Friday $25^{\text {th }}$ of April 2014 , at 10.00 hours \\ by

\section{Inge Timmers}

Born on $3^{\text {rd }}$ of July 1987 in Sittard 


\section{Promotores}

Prof. Dr. B.M. Jansma

Prof. Dr. L.J.I. Zimmermann

\section{Copromotor}

Dr. M.E. Rubio-Gozalbo

\section{Assessment committee}

Prof. Dr. E. Formisano (chair)

Prof. Dr. G.T. Berry (Children's Hospital Boston, Boston, USA)

Dr. M. Bonte

Dr. V. van de Ven

Prof. Dr. J.S.H. Vles 
Chapter 1 General introduction | Language production and working memory in classic galactosemia from a cognitive neuroscience perspective: future research directions

Chapter 2 Temporal characteristics of online syntactic sentence 29 planning: an event-related potential study

Chapter 3 From mind to mouth: event related potentials of 57 sentence production in classic galactosemia

Chapter 4 The functional neural network of sentence production 85 delineates into syntactic and motor speech planning networks: an fMRI connectivity study

Chapter 5 Neural networks related to syntactic and motor speech 99 planning are affected in classic galactosemia

Chapter 6 Assessing white matter microstructure in classic 123 galactosemia using neurite orientation dispersion and density imaging

Chapter 7 General Discussion

Abbreviations 155

Reference list

Summary

Samenvatting

Acknowledgments | Dankwoord 185

Curriculum vitae

Publication list 


$$
\omega
$$


PROLOGUE AND OUTLINE OF THE THESIS<smiles>CCCCCC</smiles> 
entral in this thesis is the study of classic galactosemia - an inborn error of galactose metabolism. We used a cognitive neuroscience perspective to investigate neural correlates of language production impairments in patients with this disease, as these are one of the most prevailing cognitive complications [see Chapter 1 for an introduction on the topic and the approach].

New-borns with classic galactosemia show an acute, potentially life-threatening syndrome after the ingestion of milk - the main source of dietary galactose. A galactose-restricted diet quickly resolves this syndrome, but cannot prevent complications on the long term. Among these complications are ovarian dysfunction, aberrant bone metabolism, and neurological and cognitive impairments.

Although several studies have characterized the cognitive impairments and studied the brain in classic galactosemia, until now there has not been a clear relation between brain abnormalities and resulting cognitive impairments. In this thesis, I have aimed to provide and characterize this relation by studying the brain online - during active task performance. In particular, I have used a language production paradigm in which overt speech is elicited in a relatively natural manner, and applied this both during electrophysiological recordings and during functional magnetic resonance imaging. Adolescent patients with classic galactosemia were compared with a healthy control group in order to find potential deviations in brain activity patterns, both in the temporal (electroencephalography, EEG) [see Chapter 3] and in the spatial domain (functional magnetic resonance imaging, fMRI, and related techniques) [see Chapter 5]. In addition, the white matter microstructure was assessed in patients with classic galactosemia, as compared to the healthy controls [see Chapter 6].

Moreover, I was privileged to explore another of my interests: the working of the healthy brain. This parallel work offered a working model of how the healthy adult brain performs the active language production task [see Chapter 2 and 4], in order to investigate in which respects patients with classic galactosemia potentially deviate.

You will find the results of these efforts in the upcoming chapters, in which a chapter on findings in patients with galactosemia is preceded by a chapter on performance of healthy adults. The dissertation starts with a general introduction in which we introduced the inborn error classic galactosemia, the perspectives and methods used in cognitive neuroscience, and how they can be combined to investigate language production impairments in this disease [Chapter 1]. 
After reading this thesis, I hope the reader will understand the need for this specific multidisciplinary research, but also that this work will be taken as an example on how to bridge two very different fields to combine expertise and yield comprehensive and extensive knowledge.

\section{Aims of the thesis}

Chapter 1 In a general introduction the disease classic galactosemia and the resulting cognitive complications are introduced. In addition, the chapter describes language production and working memory models and methods used in cognitive neuroscience, and how these can be applied to investigate classic galactosemia.

Chapter 2 Healthy adults are studied using electro-encephalography (EEG) and its derivative event-related potentials (ERPs) to investigate the time course of syntactic planning during sentence production.

Chapter 3 Syntactic sentence production is studied in patients with classic galactosemia using EEG/ERP, and compared to healthy ageand gender-matched controls to examine potential deviations.

Chapter $4 \quad$ Functional neural networks involved in sentence planning are investigated in healthy adults, using functional magnetic resonance imaging (fMRI) and functional connectivity

Chapter 5 Patients with classic galactosemia and matched controls are studied using fMRI while performing a sentence production task, to examine potential deviations in involved functional neural networks.

Chapter 6 Diffusion weighted imaging is used to investigate potential abnormalities in white matter microstructure in patients with classic galactosemia, as compared to matched healthy controls. In addition, correlations with disease variables and with behavioural outcome are examined.

Chapter 7 In a general discussion, the most important results and conclusions of the studies presented in this thesis are discussed. Furthermore, parallels are drawn across results and future perspectives are considered. 


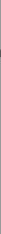




\section{CHAPTER 1 \\ GENERAL INTRODUCTION}

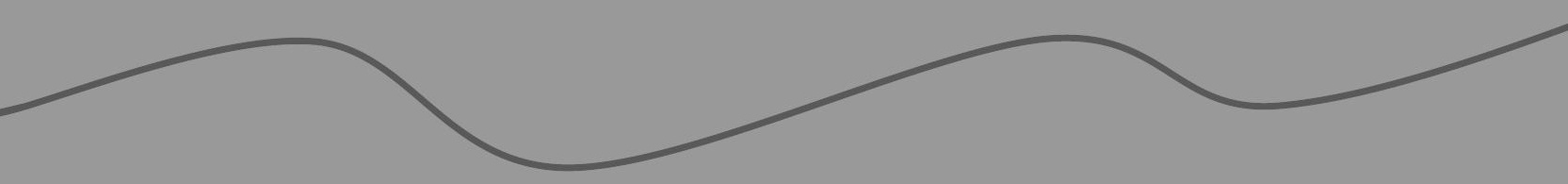

Inge Timmers, Job van den Hurk, Francsco Di Salle, M Estela Rubio-Gozalbo, and Bernadette M Jansma (2011). Language production and working memory in classic galactosemia from a cognitive neuroscience perspective: future research directions, Journal of Inherited Metabolic Disease, 34(2), 367-376. 


\section{Summary}

Most humans are social beings and we express our thoughts and feelings through language. In contrast to the ease with which we speak, the underlying cognitive and neural processes of language production are fairly complex and still little understood. In the hereditary metabolic disease classic galactosemia, failures in language production processes are among the most reported difficulties. It is unclear, however, what the underlying neural cause of this cognitive problem is. Modern brain imaging techniques allow us to look into the brain of a thinking patient online - while she or he is performing a task, such as speaking. We can measure neural activity related to the output side of a process indirectly (e.g., articulation). But most importantly, we can look into the planning phase prior to an overt response, hence tapping into subcomponents of speech planning. These components include verbal memory, intention to speak, and the planning of meaning, syntax, and phonology. This paper briefly introduces cognitive theories on language production and methods used in cognitive neuroscience. It reviews the possibilities of applying them in experimental paradigms to investigate language production and verbal memory in galactosemia. 


\section{Introduction}

In normal healthy adults, speaking is fast, automatic, and nearly perfect. Only 1 word out of a 1000 goes wrong under normal circumstances. We speak to others, but we also speak to ourselves, continuously constructing streams of verbal thoughts. Only in the rare cases in which our speech production fails, we become aware of the speaking process and our errors. In contrast to the ease with which we speak, the underlying cognitive and neural process of language production is fairly complex and not fully understood yet. Especially when patients are impaired in speech and language, it is a scientific and clinical challenge to determine where in the language planning process the problem occurs. Exploring the nature and finding the cause of the problem, however, is of relevance for tailored treatment. Motor related problems should be treated differently than deficits of verbal working memory capacity, or the access to meaning and sounds of words, or syntactic skills.

Research from different fields such as Medicine, Psychology, Psycholinguistics, Neuropsychology, and Cognitive Neuroscience are working together to form a better understanding of the normal language system, but also of language disorders. Here we focus on the possibilities to join forces with regard to language impairments related to classic galactosemia. Classic galactosemia is a hereditary metabolic disease. There is a deficiency of the galactose-1phosphate uridyl transferase (GALT) enzyme, active in galactose metabolism (Holton et al., 2001). In the acute state, neonates present with lethargy, vomiting, diarrhea, failure to thrive, and jaundice. When treatment is started, a lifelong galactose-restricted diet, the acute symptoms are relieved. However, despite diet the ovaries and the brain remain affected (Gubbels et al., 2008; Rubio-Gozalbo et al., 2010; Nelson et al., 1992; Dubroff et al., 2008). Osteopenia is also found (Panis et al., 2004), however, whether this is related to the disease itself or to acquired calcium and vitamin deficiencies (e.g., vitamin D and $\mathrm{K}$ ) is not yet clear. Among the cognitive problems that are experienced in classic galactosemia are lowered intelligence levels, memory problems and impaired language and speech (Antshel et al., 2004; Schweitzer et al., 1993; Waggoner et al., 1990). Most interestingly, and not at all understood, is that these cognitive impairments are developing in a child, despite the adequate dietary treatment. Abnormalities in speech and language have been estimated to be present in $38-88 \%$ of the patients (Hughes et al., 2009; Potter et al., 2008; Robertson et al., 2000; Schweitzer et al., 1993; Waggoner et al., 1990; Waisbren et al., 1983), although methods and samples differ in these studies. Problems are mainly said to be in expressive language. Receptive language is relatively unaffected, especially when intelligence problems are mild (Potter et 
al., 2008). There is still debate whether the problems are caused by cognitive failures or by a more sensory proprioceptive or motor failure, such as childhood apraxia of speech (CAS) (see also Potter et al., 2008, for a discussion on the CAS diagnosis). Related to CAS, it has been suggested that the observed impairment is an articulation deficit. This interpretation implies that the language disorder in these children is related to difficulties in the output phase of the language system. On the other hand, as the child acquires expressive language, the speech deficit could also be related to diverse stages in the neurocognitive preparation phase of speaking. According to language theories, these stages concern the selection of meaningful concepts to be expressed, their transfer into a meaningful grammatically well-structured message, and phonological encoding of phonemes and syllables. In addition, third factors may play a role, such as general cognitive skills (IQ) or working memory as they may hamper planning and articulation directly or indirectly.

Until now, the cognitive problems in galactosemia have been described by expert speech therapists and neuropsychological testing (e.g., Antshel et al., 2004; Potter et al., 2008). These offline instruments have the advantage of high feasibility. They are very informative, but also have the drawback of response bias, related to psychological factors and to response skills. A new and additional perspective in the study of galactosemia cognition comes from cognitive neuroscience. This field integrates knowledge on cognition with expertise in neuroscience and brain research. Using highly advanced techniques, brain activation related to cognitive function can be measured online, while the patient or healthy volunteer performs the task of interest. These methods allow us to track the neural activity related to the entire language planning process, from the intention to speak to articulation. By applying these techniques to classic galactosemia, one can objectively learn where and when in the neural system the difficulties occur along the information processing pathway. Findings could be useful for tailoring speech and other therapies for children with galactosemia. Now, speech therapy is primarily focused on improving articulation and less or not at all on language planning, such as practicing the construction of sentences. In a next step, the findings should contribute to the understanding of the underlying neural deficit. The detected brain regions of interest should be investigated at a micro level (e.g., in terms of metabolic effects per regions, or activation patterns at a cortical column level) to unravel the neural mechanisms that alter neural coding in galactosemia.

Towards a working hypothesis In 2007, we studied the medical records of 22 Dutch children with galactosemia (unpublished data). The cohort consisted of 11 males and 11 females, age at the time of testing ranged from $2 ; 1$ to $18 ; 0$. All 
children adhered to the diet, 13 had received speech therapy at one point in their lives (59\%) and 15 have (had) special educational programs (68\%). Reports from clinical experts were reviewed for relevant data on speech and language functioning. Next to articulatory problems (in $45 \%$ of the cases), these experts reported significant difficulties with sentence production in the patients (in $64 \%$ of the cases). Sentence production difficulties were described as making syntactic errors in sentences, producing sentences that were inappropriately short for their age, or producing incomplete sentences. Based on this observation, one working hypothesis is that patients with galactosemia suffer from a syntactical planning impairment, resulting in difficulties in sentence production. In addition to the language system itself, working memory is involved in language production as well and short sentences might be the result of such a verbal working memory problem (see Baddeley, 1992; 2000, for working memory models; see Baddeley, 2003, for the relation between language and working memory; Bock, 1995; Bock and Levelt, 1994, for syntactic production; Hagoort, 2005, for the general psycholinguistic model and its link to underlying brain function). Experimental paradigms can be applied to test whether the observed language impairment in classic galactosemia is related to malfunction within specific language expert systems or whether language deficits are a result of a limitation in verbal working memory.

\section{Methods used in cognitive neuroscience}

The cognitive neuroscience techniques can generally be classified based on temporal and spatial resolution. A high temporal resolution method is electroencephalography (EEG) and its derivative event related potential (ERP). In order to observe neural activity for one specific cognitive process, the EEG has to be time-locked to the onset of the stimulus or task by averaging the EEG signal in a time window around this moment of interest, resulting in the event related potential (ERP). Using ERP, one is able to look into a brain process of interest at a milliseconds time scale, which is very relevant for the study of cognition, in general a very fast information process. The planning of a word in a sentence, for example, takes about $600 \mathrm{~ms}$ from intention to articulation. Many ERP components (e.g., for perception, attention, memory, language planning, and execution) have been studied and characterized by their onset or peak latency, peak amplitude, and by their distribution across the scalp (Coles and Rugg, 1995; Luck, 2005). For an overview on language components, see Kutas and Schmitt (2003). High spatial resolution methods, on the other hand, such as functional Magnetic Resonance Imaging (fMRI), are able to locate networks involved in cognition (e.g., the language network), and to define functional 
distinct regions of interest within the network. In addition, they can provide information about structural and functional connectivity within the network. Diffusion tensor imaging (DTI) is a technique related to MRI that allows for tracking fibres of white matter, through which neural signals travel. Complementary to MRI, Magnetic Resonance Spectroscopy (MRS) is able to determine the concentration of brain metabolites, making it possible to investigate the biochemical profile of pathophysiological processes within areas imaged (Gujar et al., 2005). Together these methods deliver a great precision in unravelling the cognitive information processing within a neural network.

\section{Towards paradigms to study language production and working memory in galactosemia}

\section{Language production}

The boxes in Figure 1.1 depict the cognitive 'stages' of speaking. According to Levelt et al. $(1989 ; 1999)$ and Bock (1995), speaking begins with a planning of a message, i.e., the activation of a concept of what one wants to convey to a listener. This message can come to mind driven by various stimuli or ideas. After visual encoding of a scene or picture and after creating the concept (both still preverbal), the meaning of the message has to be encoded (semantic encoding). The next process is syntactic encoding where grammatical roles (e.g., subject versus object) and syntactical functions (e.g., tense) are assigned. These elements are assembled into a syntactic frame. In addition, various sorts of information about the word form are activated (e.g., morphemes such as preor suffixes). Finally, the phonological encoding can take place where the sound structure is determined in the form of phonological frames. When the planning of these elements is finished, the output is sent to the (pre)motor cortex to activate speech muscles to move the tongue and jaw, which allow proper articulation. In addition, we have the ability to monitor the process. This process screens the output from the speech production process for errors, and makes us overtly correct a slip. It can also work without us being aware of it within each of the expert systems (see Postma, 2000, for a review). In a nutshell, Figure 1.1 summarizes major findings from preceding research in language production. The numbers in the boxes of Figure 1.1 refer to the time course, or speed, of information processing across stages. They reflect time windows in milliseconds starting from visual picture onset and are estimated via EEG. These numbers have recently been supported by Sahin et al. (2009) using invasive intracranial electrophysiological (ICE) recording. 
The language system has also been described with regard to the brain regions sensitive to language production (see Indefrey and Levelt, 2004, for a metaanalysis). The coloured regions superimposed on a standard brain in Figure 1.1 depict the language system. As can be seen in this figure, the areas involved in language production are widespread. Parts of the temporal lobe are for instance involved in error monitoring and semantics. The parietal area (inferior parietal gyrus) is implicated in phonology. However, Broca's area (or BA 44/45) and the inferior frontal gyrus (IFG) have been found to be involved in semantics, syntax and phonology processes (see overview in Hagoort, 2005; Sahin et al., 2009). It has been suggested that the left IFG is the site where the assembly or unification takes place after the relevant information has been recruited from other brain areas (Hagoort, 2005), while the information is recruited from mid temporal areas (Snijders et al., 2009). The information is assumed to travel from the temporo-parietal areas through the arcuate fasciculus (i.e., a neural pathway of white matter fibres) to the frontal areas (Catani and Mesulam, 2008; Rilling et al., 2008).
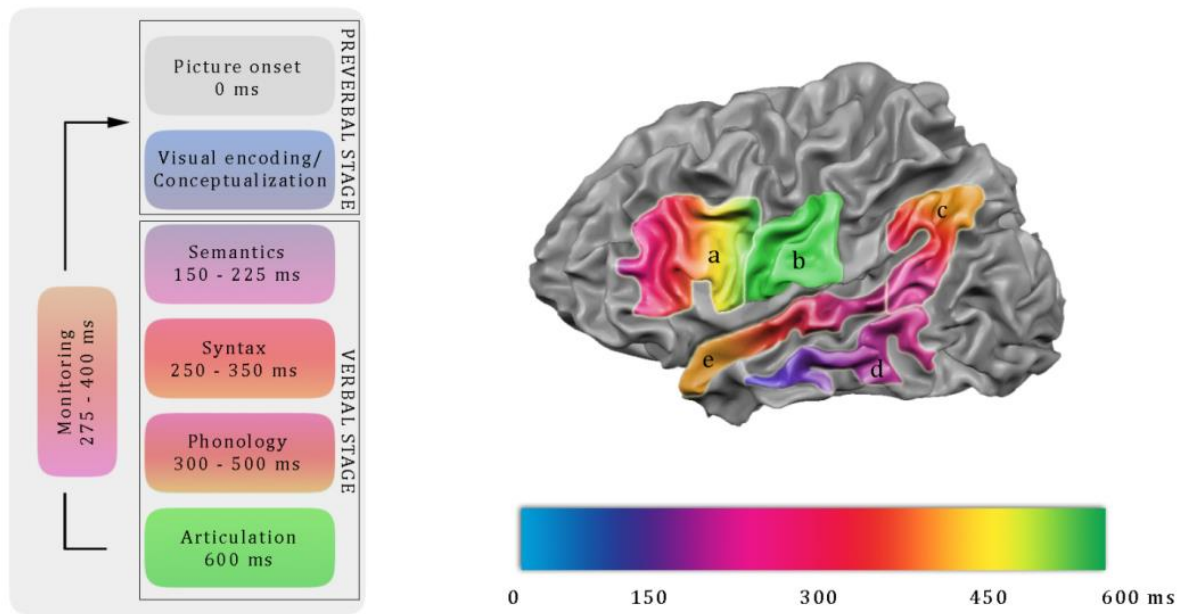

Figure 1.1. Speech production model. Displayed are cognitive stages (left) and brain areas (right) involved in language production. The numbers in the boxes represent estimates of temporal encoding for each type of information. After picture presentation $(0 \mathrm{~ms})$, the visual system encodes the stimulus and activates a preverbal concept. The appropriate lexical entries are selected (150$225 \mathrm{~ms}$, middle temporal gyrus (d)). The next stage involves syntactic encoding (left inferior frontal gyrus (IFG), taking place around 250-350 ms post stimulus (a)). Finally, phonological encoding takes place (300-500 ms, posterior superior temporal gyrus, angular gyrus (c)). The message is then presumably assembled in the left IFG. After all planning has taken place, the finished speech plan is sent to (pre-) motor areas (b) to be prepared for articulation. An online self-monitoring feedback loop (275-400 ms, superior temporal gyrus (e)) is capable of keeping track of the speech production process and intervenes if required. It has to be noted that boxes or stages are for display purpose only. Speech production does not involve encapsulated modules, but involves several brain regions 
that interact in a cascading manner. (Model adapted from Indefrey and Levelt, 2004, plus recent temporal information, for example Sahin et al., 2009)

In order to study language production in classic galactosemia from this perspective, we propose example paradigms with focus on core processes, such as conceptualization, syntactic encoding and verbal working memory. A summary of the paradigms to be used is given in Table 1.1. This is a novel multidisciplinary approach that aims to provide new insights in the cognitive pathophysiological processes in galactosemia.

Observed differences (as compared to healthy subjects) in an experiment that taps conceptualization could be interpreted as a conceptualization deficit in the patient group. As conceptualization is input for further language encoding stages, the deficit in turn can cause subsequent problems in following stages. A lack of effect during a paradigm tapping conceptualization means that the observed language deficit in the patients may be attributed to later processing stages, after conceptualization took place. A difference in neural activity (as compared to healthy subjects) during a semantic encoding paradigm would point to impaired semantic processing in the patients. However, one can conclude a semantic deficit only in case the conceptualization account is ruled out for reasons expressed above. No differences between the groups with regard to semantic planning would indicate proper semantic encoding. Using this approach the different stages of language can be tested. One should keep in mind, finally, that any suggested impairment in a specific language process in galactosemia, necessitates excluding a verbal working memory deficit. There is a close interaction between language production systems and verbal working memory. In the context of galactosemia, it has to be investigated whether patients with galactosemia have difficulties with either one of these functions or perhaps with both. However, it is very difficult to disentangle what is attributable to an inability to maintain verbal information, for instance during syntactical encoding, or to an inability of syntactical encoding per se.

Furthermore, it should be noted that the translation of experimental paradigms from healthy study subjects to patients with galactosemia is not straightforward. As was described, many patients have a lower intelligence and are, a priori, expected to have difficulties with mastering complex paradigms. Therefore, the paradigms have to be simplified such that even the lower functioning patients can perform them, or these patients should be excluded from the study group. In practice, there should be a balance between designing relatively simple paradigms and recruiting patients with intellectual abilities that allow executing 


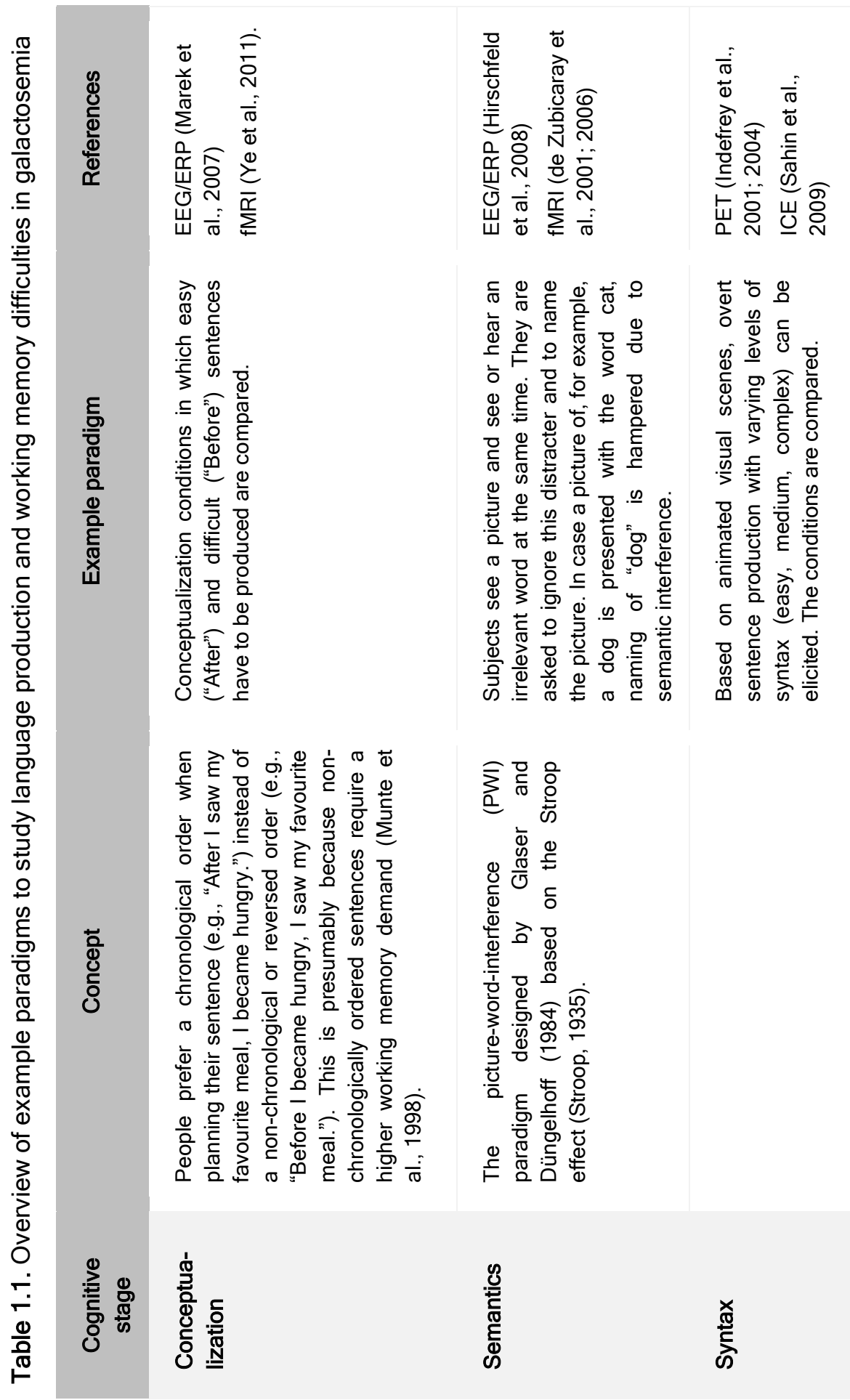




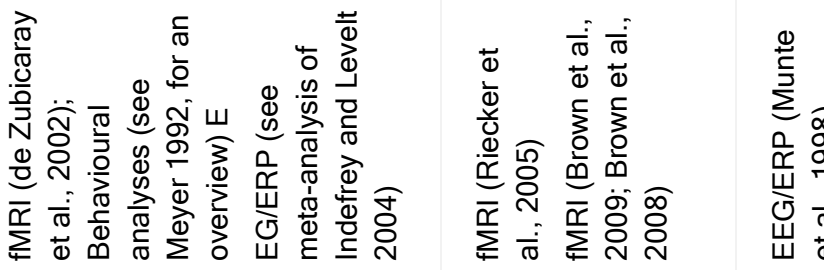

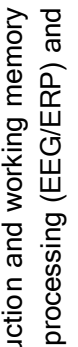

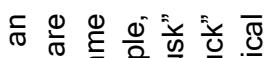

市 শ

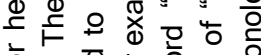

ᄒ⿱亠

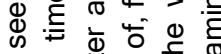

ర

त

总怘

চ

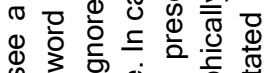

ஸ

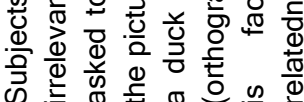

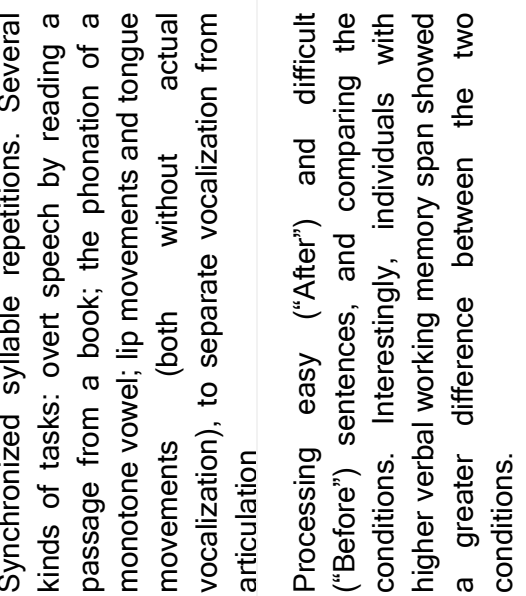

흔 응

ธึํํ

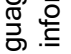

它

蒙

㐫 ठ

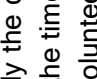

중

फ़

$\div$ 을

बं山

ํำ

बे

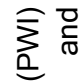

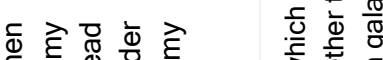

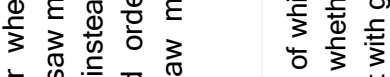

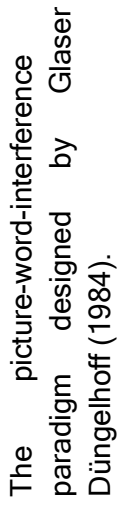

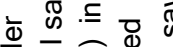

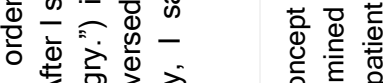

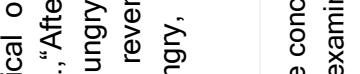

응

응

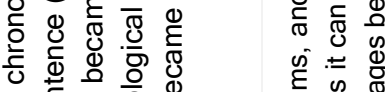

๑ क

क元

屯

人

๑.

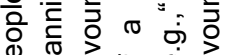

வ $\frac{\pi}{0} \underset{0}{\mathbb{6}}$

함
음
음
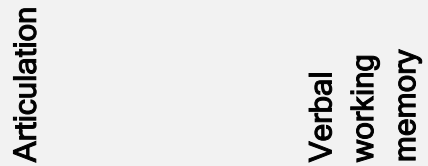

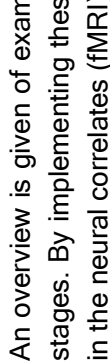


the paradigms. When patients with a too low IQ (lower than 80) are excluded, the paradigms described in Table 1.1 all are expected to be feasible in the patient group. Of special interest in language production for galactosemia is the study of syntactic encoding. Our group has performed an ERP study using an adapted version of the Indefrey et al. experiment $(2001 ; 2003)$ in a group of 20 healthy subjects and in 9 adult patients with galactosemia. The aim was to identify the ERP components related to syntactical encoding. Preliminary results indicate components of interest in our ERP data comparable to the findings of Sahin et al.'s ICE study, where distinct neural activity for semantic, grammatical and phonological encoding was found at around 200, 320 and $450 \mathrm{~ms}$ after target word onset, respectively, at the place of the electrode inserts (BA44/45 or Broca's area). A descriptive comparison of the data can be found in Figure 1.2. Further, our findings support the claim that the IFG is not only involved in syntax (as was found by the PET study of Indefrey et al., 2001; 2003), but also in conceptualization and/or semantic processing. For galactosemia research, this means that the IFG is an area of special interest for further investigations. Preliminary results in the nine galactosemia adults suggest that language production processes in galactosemia might be delayed (i.e., delayed onset latencies of the waveforms) in addition to amplitude differences.
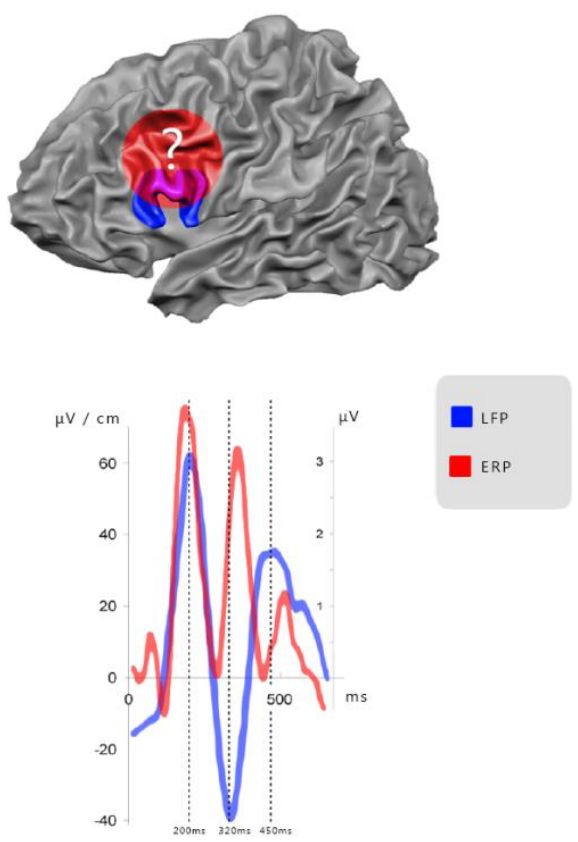

Figure 1.2. Local field potentials (LFPs) versus ERPs during syntactical encoding. A descriptive comparison is made between the intracranial local field potentials of Sahin et al. (2009) and the extracranial EEG/ERP study of our group. Both studies investigated the brain's response to the 
encoding of syntax. Sahin et al. instructed their participants to make grammatical inflections while our participants were asked to utter a complete sentence in response to an animated scene. Lower panel: Overlay of average LFP and ERP within the same time scale. Interestingly, despite the differences in the method and in the syntactic task, the morphology of the waveforms is strikingly similar in the target peak latencies (200, 320, $450 \mathrm{~ms})$. Granting the assumption that peaks in LFP and ERP signal reflect maximal neural activity, this descriptive comparison suggests common aspects in the two signal types for language encoding. Upper panel: The brain area depicted in blue represents Broca's area, i.e., the location of the intracranial recording. The red circle reflects the presumed source of the EEG data (in correspondence with the PET study results of Indefrey et al., $2001 ; 2003$, using the same paradigm). The EEG source still has to be confirmed.

\section{Working memory}

The most widely used model of working memory comes from Baddeley (1992; 2000). In this model, working memory consists of multiple components (e.g., a central executive supervisory system; and an episodic buffer, a system proposed to link information forming one unified whole, such as in a movie scene). Working memory components have been described in terms of brain areas and networks (e.g., Cabeza and Nyberg, 2000). So far, not much is known on syntactic verbal memory. A suggestion is that the inferior frontal gyrus (IFG or BA44) plays a relevant role in assembling strings of information - may that be phonological, syntactic, or semantic - into a meaningful message (Hagoort, 2005). If one compares the language production network (Figure 1.1) with the working memory network (Figure 1.3), one can see that there is an overlap, especially in the IFG. Similar to the proposed function of the IFG in language production (selection and assembly or unification), this area is proposed to be involved in selection, retrieving and maintaining verbal information (Martin and Chao, 2001), with syntactic retrieval from mid temporal lobes (Snijders et al., 2009). This view suggests that there is a close link between the language and the verbal working memory system.

\section{Linking the multiple disciplines}

Although it is very important to specify where and when failures occur during language production and working memory performance in patients with classic galactosemia, it might be even more important to understand why they occur. To understand the changes or deficits in neural information processing, it is important to study the specific data transfer and metabolic processes that affect information processing in pre-specified brain regions of interest to see how these specific cognitive problems result from the deficiency in galactosemia. 

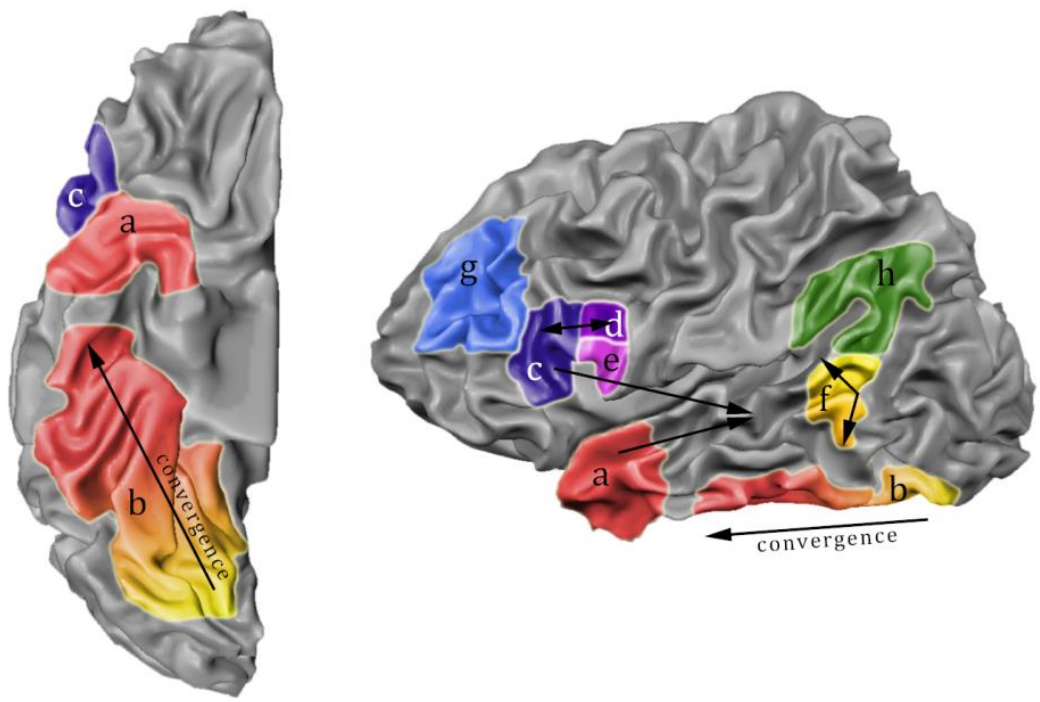

Figure 1.3. Working memory model. The anterior temporal pole (a) is believed to play an important role in semantic memory retrieval and representation of specific semantic items. Regions in the fusiform gyrus (b) have proven to show differential responses to different categories of objects, converging in specificity from posterior to anterior regions. The inferior frontal gyrus (IFG) (c, d, e) is involved in several (semantic) working memory related tasks: rehearsal (c), selection (d) and production (e). The lateral temporal cortex (f) is related to the perception of motion, of both biological (dorsal) and artificial (ventral) objects, and to lexical memory, whereas the posterior superior temporal gyrus (h) is the presumed region where phonologic loops are maintained. Finally, dorsolateral prefrontal cortex (dIPFC) (g) has an overall executive role in working memory tasks (after Cabeza et al., 2002; and Martin and Chao, 2001). Obvious is the overlap of this memory network with the language network depicted in Figure 1.1.

The pathogenic mechanisms in classic galactosemia and how they result in the cognitive impairments despite diet, are not entirely clear. Possibly, the damage has already occurred in utero, or in the first few days of life before the diet is introduced. Normally, galactose is converted into galactose-1-phosphate (gal-1P), further metabolized into glucose-1-phosphate by GALT (i.e., the Leloir pathway for galactose metabolism). Because of the GALT deficiency in galactosemia, there is an accumulation of gal-1-P and galactitol, which is considered to be one of the most important factors in the pathophysiology. Apart from the toxicity of these metabolites for body tissue and organs, the accumulation is also believed to result in inositol abnormalities (see Berry, 2011) and aberrant synthesis of glycoproteins and glycolipids, which are important building stones, for instance, for myelin. Myelin is a relevant component for functional data transfer between neurons and brain regions. Indeed, early anatomical MRI scans show abnormal white matter patterns in 
patients with galactosemia, suggested to be due to abnormal myelin (Nelson et al., 1992), implying hampered data transfer. Both language production and working memory are carried out in specific networks (see Figures 1.1 and 1.3, respectively) that partly overlap with each other (both anatomically and possibly functionally). If there is a direct link between myelin degradation and brain function, one could expect that myelin is relatively more abnormal within these target areas. Studies with high resolution acquisition and advanced analysing techniques will have to point out whether the specific functional areas are affected. One such approach could be Diffusion Tensor Imaging (DTI), a measure that is strongly linked to axonal and myelin integrity, and has proven a very sensible and early finding in several asymptomatic brain diseases (Nierenberg et al., 2005; Reading et al., 2005). In its more sophisticated application, DTI can even provide detailed information regarding anatomical connectivity, and will probably be able to indicate whether it diverges between patients with galactosemia and healthy controls within the language and memory network. The studies that have already been done in galactosemia (Kaufman et al., 1995; Nelson et al., 1992) did not show clear regional differences, nor correlations with cognitive processes, based on the available data (low resolution 0.5-1.5 Tesla scanner). The availability of higher resolution scanner in the future allows investigating a functional link between myelin and cognition. However, one interesting finding of Nelson et al. is that about onethird of the patients had mild lateral ventricle enlargement and clustering of white matter lesions around the lateral ventricles. Fascinating about this report is that the arcuate fasciculus, the white matter bundle connecting temporoparietal language areas with the frontal language areas, is located alongside of the lateral ventricle. Abnormal myelin might affect the information flow within the networks and thus might affect language function. A target method of choice to investigate differences in such information flow within neural circuits, such as the arcuate fasciculus, in patients with galactosemia versus healthy controls would be functional connectivity analysis.

Recently, it has been suggested that epigenetic factors may be involved in the pathology of galactosemia. Coman et al. (2010) studied gene expression profiles of four patients with galactosemia. They identified several up- or downregulations in gene expressions in these patients. Genes involved in cell signalling pathways, such as the mitogen-activated protein kinase (MAPK) signalling and the calcium signalling pathway, both implicated in neural signalling processes, showed different expression patterns. The most dysregulated gene was Septin 4, of which the expression was decreased 85fold. Septins are proteins that are involved in a large number of cellular functions, such as membrane dynamics, cytokinesis, vesicle trafficking, 
exocytosis, and apoptosis (Cao et al., 2009; Haller et al., 2005). Septin 4 (or SEPT4) proteins have been implicated in neurodegenerative diseases, such as Alzheimer's disease or Parkinson's disease. It is expressed in all human tissue, but shows an high expression in the brain (Haller et al., 2005). Further studies will be necessary to elucidate whether these genes are relevant for the origin of the chronic complications. One of the possibilities might be to use ultra-high field imaging. With this method, the detection of proteins by producing specifically tailored contrast mechanisms, e.g., by the use of immunoconjugated magnetic nanoparticles (Hilger et al., 2007), might become possible in the future. This in turn might permit to quantify the density of specific substances, among which Septin 4, which can be linked to specific brain regions of functional interest, such as memory or language.

It would be intriguing to examine whether genes encoding for cognitive functions are differentially expressed in galactosemia. One such gene is the FOXP2 transcription factor gene, which has been implicated in speech and language disabilities (Enard et al., 2002; Fisher and Scharff, 2009). Such a research would provide another missing link: the link between the genes and behavioural level. Ultimately and ideally, in the future a multidisciplinary approach in combining genes, gene expressions, protein imaging, and cognition in galactosemia should result in a working model that explains the cognitive complications observed in galactosemia.

\section{Conclusions}

A novel and innovative approach is suggested to bridge the gap in disciplines between the behavioural level on the one hand and the metabolic and cellular level on the other hand, in the context of the hereditary metabolic disease classic galactosemia. More specifically, we propose to study language production difficulties in patients with galactosemia from the perspective of cognitive neuroscience and to correlate language behaviour with brain functions, connectivity, and metabolism. Experimental paradigms from language production research and highly advanced techniques allow studying the brain functions of a patient online. This approach has the major advantage that not only the output stage of language production can be studied, but the preceding planning stages as well. This is necessary as language production is a fairly complex process consisting of several subcomponents, referred to as conceptualization, semantic, syntactic, and phonological encoding, followed by articulation. It is highly relevant to learn exactly where and when in the cognitive and neural system of language the difficulties arise in classic galactosemia to be able to offer for example speech therapy in a tailored manner. In addition to 
language, verbal working memory is highly involved in language production processes and vice versa. Next to describing the cognitive deficits in terms of timing (ERP) and locations (fMRI), connectivity analysis and the analysis of metabolites related to functions are relevant to understand the underlying neural processing deficit. The aim of applying these methods and accompanying paradigms in galactosemia research - an approach that has never been taken before - is to gain more insight in the cognitive pathophysiological processes in galactosemia. 


CHAPTER 2

\section{TEMPORAL CHARACTERISTICS OF ONLINE SYNTACTIC SENTENCE PLANNING: AN EVENT- RELATED POTENTIAL STUDY}




\section{Summary}

During sentence production, linguistic information (semantics, syntax, phonology) of words is retrieved and assembled into a meaningful utterance. There is still debate on how we assemble single words into more complex syntactic structures such as noun phrases or sentences. In the present study, event-related potentials (ERPs) were used to investigate the time course of syntactic planning. Thirty-three volunteers described visually animated scenes using naming formats that varied in syntactic complexity: from simple words ('W', e.g., "triangle", "red", "square", "green", "to fly towards"), to noun phrases ('NP', e.g., "the red triangle", "the green square", "to fly towards"), to a sentence ('S', e.g., "The red triangle flies towards the green square. "). Behaviourally, we observed an increase in errors and corrections with increasing syntactic complexity, indicating a successful experimental manipulation. In the ERPs following scene onset, syntactic complexity variations were found in a P300-like component ('S'/'NP' > 'W') and a fronto-central negativity (linear increase with syntactic complexity). In addition, the scene could display two actions unpredictable for the participant, as the disambiguation occurred only later in the animation. Time-locked to the moment of visual disambiguation of the action and thus the verb, we observed another P300 component ('S' > 'NP'/'W'). The data show for the first time evidence of sensitivity to syntactic planning within the P300 time window, time-locked to visual events critical of syntactic planning. We discuss the findings in the light of current syntactic planning views. 


\section{Introduction}

Language is an important basis for communications with others. As a speaker, we are constantly constructing streams of thoughts and planning messages to transfer these thoughts into the outside world. As a listener, we receive acoustic, visual and contextual information, and integrate this into a meaningful message. Whereas speech production and comprehension (or encoding and decoding) have been separate fields in psycholinguistics, recent discussions argue that they are interwoven, non-isolated processes that largely share underlying mechanisms (see e.g., Kempen, 2013; Pickering and Garrod, 2013). Although a lot is already known about online syntactic processing during comprehension based on electroencephalography (EEG) and functional magnetic resonance imaging (fMRI), comparably less is known for the production analogue. A balanced knowledge is necessary to investigate potential commonalities of syntactic processing in both modalities. The current study focuses on syntactic planning during production and addresses the question when in time syntactic planning for speaking takes place.

There are many accounts on how we apply grammatical rules to be able to generate meaningful utterances. In general, most researchers agree that speaking involves conceptual, syntactic, and phonological planning that leads to articulation. Views differ on whether we should see these processes as serial stages, unfolding over time, or more as parallel processes. In classic serial accounts, speakers carry out syntactic sentence planning in several steps. First, lexical concepts and corresponding syntactic information (e.g., whether it is a noun or adjective; lexical selection) are identified and activated. Secondly, syntactic relations and functions are assigned to each word (e.g., subject versus object; function assignment) and proper inflections are added (e.g., $-s$ for plural, -ed for past tense). Finally, words are assembled into so called syntactic structural frames (constituent assembly) (Bock and Levelt, 1994; Bock, 1995). Friederici $(2002 ; 2011)$ also assumes serial processing, but suggests that syntactic processes first build a local structure, after which grammatical and semantic relations are assigned in a utterance. In an interactive view, Kempen (2013) (but see also e.g., Hagoort, 2005; Vosse and Kempen, 2008) describes a localist neural network model in which grammatical encoding is a task assigned to the Unification space (or U-space). Via a recursive transition network (RTN), activation spreads across so-called treelets or syntagma's that can be bound to lemmas. A list of annotated lemmas is eventually converted to a list of word forms. The author notes, however, that although processes (conceptual, syntactic) are initiated in parallel, the behaviour of the network may seem serial because some processes may require more time. The stage-like behaviour is 
therefore only an emergent property of the model. Other views do not assume that a formal grammar (rules) interacts with a mental lexicon (words). They rather consider language as an emergent property, emphasizing the role of the user's experience (Elman, 1991; Elman, 2009; Gahl and Yu, 2006; Janssen and Barber, 2012; Langacker, 1987; Tomasello, 2005). The role of experience, however, is also evident in other, more classic views (e.g., the recursiveness of network models, Kempen, 2013).

Most theories envision speech production as an incremental process, although the units of increment differ between views (Allum and Wheeldon, 2007; Allum and Wheeldon, 2009; Bock, 1995; Levelt, 1989), but might also vary across speakers (e.g., cognitive capacity, experience), and could be dependent on the situational context (e.g., time pressure) (Bock, 1995; Ferreira, 1991; Meyer, 1996; Wagner et al., 2010). Further, sentence planning can be either lexically or structurally incremental (one can guide the other, Bock et al., 2004), or a flexible interaction between both. Evidence from a recent study points towards structural incrementality (Konopka, 2012), implying a role for structural assembly in early sentence production (i.e., preceding lexical retrieval; and in contrast to psycholinguistic views in which lexical retrieval occurs prior to syntactic planning; Bock and Levelt, 1994; Levelt, 1989).

Whereas there is still the ongoing debate about the exact nature of syntactic planning, only recently studies have ventured to investigate the neural aspects of information processing during the production of complete sentences. Several brain areas have been reported to be involved in syntactic encoding, including the left inferior frontal gyrus (IFG; BA 44/45/47), left posterior middle temporal gyrus (MTG, BA 21), and bilateral supplementary motor areas (SMA; BA 6) (Haller et al., 2005; Hickok, 2009; Indefrey et al., 2001; Indefrey et al., 2004; Menenti et al., 2011; Price, 2010; Segaert et al., 2011). Not much is known, however, about the time course of syntactic encoding (see also Ganushchak et al., 2011). A method of choice to investigate temporal characteristics of information access is EEG, and its derivative, the event-related potential (ERP). For single word production, the experimentally elicited lateralized readiness potential (LRP) (van Turennout et al., 1997; 1998; 1999), and the N200 go-no go component (Schmitt et al., 2000; 2001a; 2001b) have been extensively studied in single word and noun phrase production. Based on the LRP and the N200 go-no go results, it has been estimated that semantic access precedes syntactic access by approximately $90 \mathrm{~ms}$, which is followed by phonological encoding after around $40 \mathrm{~ms}$, suggesting incremental planning (but also see the discussion in Rahman et al. 2003). So far, the most direct measure of the time course of syntactic encoding was carried out via invasive intracranial 
electrophysiology (ICE). Sahin et al. (2009) used ICE to record local field potentials (LFPs) near Broca's area in patients who had to either read or inflect a word (past/presence or singular/plural). The recordings revealed a component around $320 \mathrm{~ms}$ after target word presentation sensitive to (morpho)syntactic processing. In an ERP study, Marek et al. (2007) asked participants to overtly describe a walk through a 2D grid consisting of geometric colour figures either in a simple ("go up, go right"), medium ("go up to the circle"), or complex ("go up to the green circle") manner. They found a P300-like component at 350-500 ms post stimulus onset, distributed over centro-parietal electrodes, that was more positive for medium and complex utterance conditions compared to the simple condition. The authors concluded that the P300 is sensitive to conceptual and/or syntactic complexity variations.

In summary, electrophysiological studies suggest that syntactic encoding is carried out around $300-500$ ms after stimulus onset. However, this conclusion is based on indirect measures (LRP/N200 go-no go paradigms), rather than direct naming; based on rather artificial naming tasks (explicit inflection of a certain word within sentence context - which we normally do not do in an highly automatic process); or based on ambiguous interpretations of the data (i.e., no clear separation of conceptualisation and syntactic complexity in the experimental design). In the present study, we used a more direct and natural approach, in order to gain insights into the electrophysiological correlates of syntactic planning. In analogy to a positron emission tomography (PET) study by Indefrey et al. (2001; 2004), we employed a paradigm where visually animated scenes elicited overt multi-word utterances in a relatively natural way. Participants were instructed to describe the scenes as fast and accurate as possible using a sentence -, a noun phrase -, or a single word format (in Dutch). For example, in one of the visual stimulations a red triangle bumps into a green square. In the complex, sentence-leve/ ('S') syntax condition participants would describe the scene as "De rode driehoek botst tegen het groene vierkant op." ["The red triangle bumps into the green square."], in the medium, noun phrase level syntax ('NP') condition they would illustrate the trial as "de rode driehoek", "het groene vierkant", "tegen op botsen" ["the red triangle", "the green square", "to bump into"]. In the minimal syntax, words ('W') condition the correct response would be "driehoek", "rood", "vierkant", "groen", "tegen op botsen" ["triangle", "red", "square", "green", "to bump into"]. The participants were instructed on the type of naming format at the beginning of each block. Visual stimulation was kept constant across conditions.

The rationale of Indefrey et al. behind the three different utterance types was that the required syntactic processing parametrically varied in complexity (2001; 
2004). Overall the task requires a range of cognitive information processing. The visual scene - identical across conditions - triggers visual and conceptual encoding of motion, colour, and form, as well as of the action (either 'to fly towards' or 'to bump into'). In addition, concepts (i.e., the different geometrical figures plus the verb) must be ordered for serial articulation. Linguistic encoding, depending on the utterance instruction, should trigger the build-up of the appropriate syntactic structure and the filling in of the structure with suitable elements. Following the logic of Indefrey et al. we assumed that the ' $W$ ' condition required lexical selection of words but virtually no syntactic encoding. In the 'NP' condition, syntactic processing was necessary on a noun phrase level, because the retrieval of certain syntactic information and inflections was required (i.e., the article of a noun, inflection of the adjectives, assembly into a phrase). In the ' $S$ ' condition, syntactic planning was necessary on a sentence level, which includes the processing required in the words ' $\mathrm{W}$ ' and noun phrase 'NP' conditions, but also the combination of two noun phrases by adding the verb in its proper form.

The application of high resolution EEG allowed us to time-lock the ERP to certain events within the utterance planning process. We specify two critical events. One event is the scene onset, as it starts the planning of the first elements of the utterance. A second critical event is the moment at which the target action is disambiguated (both scene variations started identical and diverged only from that point on). At that moment, one of the two actions were displayed - unpredictable for the participant. The disambiguating visual moment allowed the speaker access to the target action concept and its syntactic realisation. It also allowed to bind the first noun phrase to the second noun phrase, using the target verb.

ERPs were recorded from the scene onset on. We took a rather explorative approach in this study. Based on a more modular, serial account, we expected that components sensitive to syntactic processing would show a parametric amplitude modulation related to the syntactic complexity variation within a certain time window (based on the additive factor logic, Sternberg, 1969). The detected time windows of the parametric modulation should give insights into the time course of syntactic planning stages. Based on the limited electrophysiological literature available (Marek et al., 2007; Sahin et al., 2009), we expected to observe a variation with syntactic complexity around 300-500 ms time window after stimulus onset in correspondence of a P300 component, associated with phrase-level syntactic planning. For post-verb disambiguation sentence planning, we aimed to present first empirical evidence with this experiment. In addition, from a more integrative theoretical view, we did not rule 
out immediate and parallel integration that would affect neural processing in a non-additive manner. This parallel processing might result in early effects in the ERP (i.e., in time windows sensitive to visual and conceptual encoding, Hillyard et al., 1998; Rabovsky et al., 2012; Rahman and Sommer, 2008). We will discuss the results in the light of the different language accounts.

\section{Materials and Methods}

\section{Ethics statement}

The ethical committee of the Faculty of Psychology and Neuroscience (Maastricht University) gave clearance for the study. All participants gave written informed consent.

\section{Participants}

Thirty-four healthy volunteers participated in this study. Data of one participant were excluded from the analysis because of the health history and current medication use. Twenty-one of the 33 remaining participants were female. One was left-handed. The mean age was 21.8 years (SD 2.6 years). All had normal or corrected to normal vision and were native Dutch speakers. The participants received financial compensation or received academic credit points.

\section{Stimuli}

Visually animated scenes were presented to the participants. Each scene consisted of three geometrical shapes (square, triangle, or circle) having one of three different colours (red, blue, and green). The individual figures covered approximately $1.6^{\circ}$ (height) of visual angle and were configured around the centre (one above and two below the centre on either side). The total configuration covered approximately $5.8^{\circ}$ (width) $\times 5.4^{\circ}$ (height) of visual angle. In each trial, one of the three geometrical figures performed an action upon another figure: it could either be 'to fly towards' or 'to bump into'. The two scene types started visually identical until they diverged at a certain point (see the Procedure section for details on how the scenes differed). In each scene two of the objects could be distinguished by their colour only. This made it more natural to name the colour together with the shape of the objects. The content of the scene was randomly varied across trials (i.e., the shapes, colours, positioning of the figures, and the action). Such variation of the events in the scene was included to keep participants alert and to have online utterance planning on a trial by trial basis. 
The paradigm was designed using Presentation 14.0 software (Neurobehavioral Systems, Inc.).

\section{Procedure}

Participants were instructed to overtly describe the presented animated scenes using one of three possible responses: word-'W', noun phrase-'NP', or sentence-'S' format (an example for each condition would be as follows: word'W' - "driehoek", "rood", "vierkant", "groen", "naar toe vliegen" ["triangle", "red", "square", "green", "to fly towards"]; noun phrase-'NP' - "de rode driehoek", "het groene vierkant", "naar toe vliegen" ["the red triangle", "the green square", "to fly towards"], and sentence-'S' - "De rode driehoek vliegt naar het groene vierkant toe." ["The red triangle flies towards the green square."]) (see the Introduction for an example of the 'to bump into' scene types). After having received instructions, a practice version consisting of 3 blocks (i.e., one per condition) containing 18 trials each was started. The practice session was followed by the main experiment, which consisted of three runs. A single run consisted of three blocks (one per naming condition). The order of naming conditions was randomized within each run (i.e., six possible run types) and across participants.

Each block started with a brief instruction reflecting the type of naming format to be performed (i.e., either 'SENTENCE', 'NOUN PHRASE', or 'WORD'), followed by 40 trials, consisting of a different scene each (see Stimuli). A total of 120 trials were recorded for each condition. Each trial started with a fixation point (white asterisk on a black background) for $2000 \mathrm{~ms}$, followed by the display of the geometric figures that moved. The duration of animation in the scene differed (955 or $1885 \mathrm{~ms}$ ), depending on the action format ('to fly towards' or 'to bump into', respectively). The difference in animation durations was due to a different amount of action frames (10 versus 18 frames, where the actual 'bump' event occurred at frame 14, at $1520 \mathrm{~ms}$ after scene onset). The two scenes types associated with the two different actions were visually identical until the moment that the 'to fly towards' trials froze while 'to bump into' trials continued. The stimulation always ended with a freeze configuration lasting $3000 \mathrm{~ms}$ (see Figure 2.1). Participants were instructed to start the description of the scene as fast and as accurate as possible, and to minimize eye movements. The next trial started via a self-paced button push (by USB keyboard key). This self-pacing format was chosen to take into account inter-individual differences in naming onset and duration. An entire trial took approximately 8000 ms (fixation, scene, freeze time and button to switch to the next trial to continue). 
During the recordings, participants were seated in an electrically-shielded, sound-attenuated room in front of a computer monitor (distance approximately $80 \mathrm{~cm}$ ).

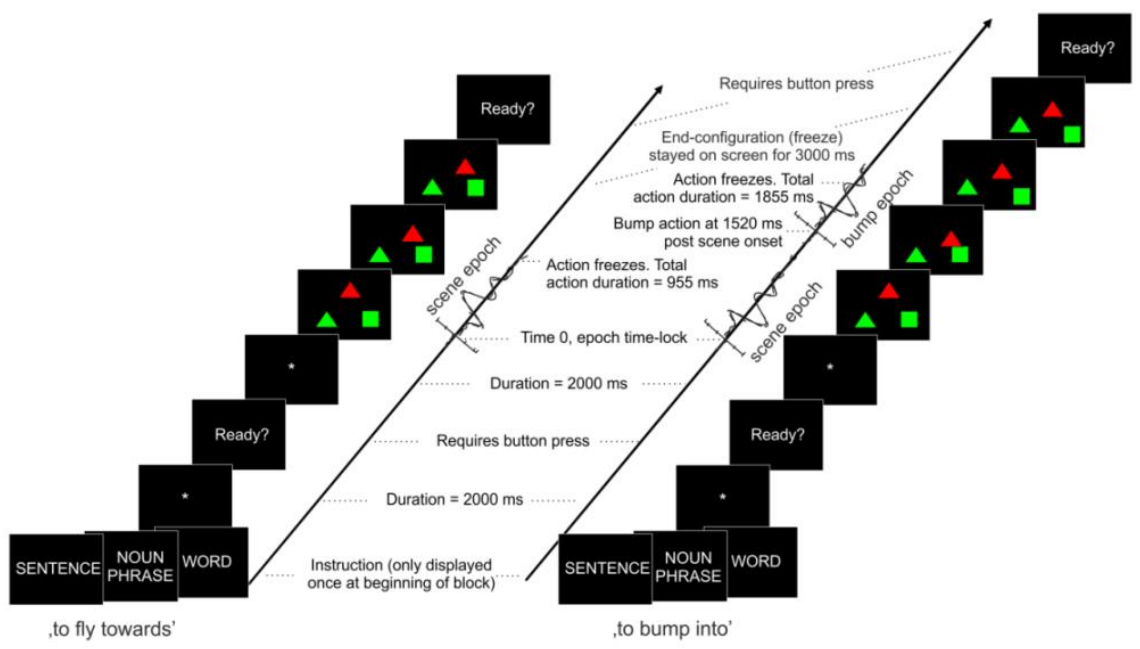

Figure 2.1. Overview of trials. Schematic overview of the experimental trials, separately for the two action formats ('to fly towards' and 'to bump into'). For illustrative purposes, only screenshots of the trials are displayed (the objects were actually moving). The displayed ERP epochs illustrate the different time windows of interest for the analysis (scene epoch, immediately starting after scene onset; and bump epoch, after the 'bump' event and hence after disambiguation of the target verb). Note that action formats are randomized across trials and are not instructed nor predictable to the participants.

\section{Apparatus and EEG recording}

The EEG was measured using an elastic cap in which 32 tin electrodes were mounted (Electro-Cap International (ECl), Inc.), positioned according to the international 10-20 system (Jasper, 1958). The signal was recorded from twenty electrodes - F3, Fz, F4, FC3, FCz, FC4, C3, Cz, C4, CP3, CPz, CP4, P3, Pz, $\mathrm{P} 4, \mathrm{O} 1, \mathrm{Oz}, \mathrm{O} 2, \mathrm{~T} 3$ and $\mathrm{T} 4$ - referenced online to the left mastoid (A1). Offline the signal was re-referenced to the average signal of both $A 1$ and $A 2$. Vertical eye movements and blinks were monitored by two electrodes placed at the left upper and the lower orbital ridge. Horizontal eye movements were recorded with electrodes placed on the left and right cantus. The impedance of all electrodes was kept below $5 \mathrm{k} \Omega$. Data acquisition was done using Brain Vision Recorder software (Brain Vision, MedCaT B.V.) and the signal was amplified using a 0.05$50 \mathrm{~Hz}$ band pass and sampled at a $2 \mathrm{~ms}$-interval $(500 \mathrm{~Hz})$. The scene onset as 
well as the voice onset triggered a code pulse. The code was written directly into the EEG recordings and was used for later event-related analyses. The voice onset was recorded via the microphone and transferred as onset time pulse into the log file. The pulse was triggered when the sound pressure level reached a certain threshold (individually adjusted for each participant).

\section{Data analyses}

For the behavioural data, the number of errors (i.e., any deviation from the expected utterance: incorrect object, colour, action, naming format, or ordering) and corrections (i.e., any overt corrective effort during the response utterance) were computed using the recorded audio data and the manual scores collected online by the experimenter. Dysfluent speech was not necessarily coded as a error or correction, only if there was overt corrective effort or a mistake. Moreover, we computed the voice onset time (VOT) as the time between the onset of the scene and the onset of the overt naming. We also calculated the total speech time (TST) that was defined as the time difference between naming onset and the button response. VOTs $<0.5$ seconds and $>4.5$ seconds and TSTs $<2$ seconds and $>10$ seconds were considered outliers and were discarded from the analysis. A repeated-measures General Linear Model (GLM) with syntactic complexity as factor (3 levels: W, NP, S) was used to analyse the behavioural data.

With respect to the EEG data, trials in which the participant's response was incorrect, corrected or absent were excluded from further analysis. The EEG data related to the correct naming trials were epoched from -200 to $2500 \mathrm{~ms}$ post stimulus onset (to include the entire interval from the onset of visual scene to the end of the display/onset of articulation), band pass filtered from $.3-30 \mathrm{~Hz}$ (zero phase, $24 \mathrm{~dB}$ ), and baseline corrected (from -200 to $0 \mathrm{~ms}$ ).

In order to reduce artefacts in the signal, Independent Component Analysis (ICA) was used. ICA blindly decomposes the multi-channel EEG data into temporally maximally independent components (which computationally corresponds to components sharing the least mutual information; Makeig and Onton, 2009; Onton et al., 2006). An Independent component (IC) is characterized by a time course and a scalp topography reflecting the contribution (weight) of that component to the EEG signal at each of the scalp channels (not to be confused with traditional ERP scalp topographies). The ICs typically consist of brain or non-brain (artefact) processes, or are comprised of noisy data (e.g., large, atypical movements do not share mutual information with the other sources and hence would fall into separate unreliable ICs). Non-brain artefact-related (e.g., stereotyped eye blinks, eye movements, and muscle 
movements) and noisy ICs can be identified by visually inspecting the corresponding topographies and time courses. By removing such ICs, one can filter out the contributions of those processes to the signal. Therefore, this procedure allows de-noising the data, without losing trials and hence statistical power.

In our procedure, we first removed (in the original EEG space) the large and atypical artefacts from the data based on visual inspection to avoid that ICA would extract unreliable ICs devoted to noisy data. On average, $82.1 \%$ of the trials were kept for further analysis: 97 trials in ' $S$ ', 98 in 'NP' and 102 in 'W' condition. Then, the data were decomposed using the infomax algorithm in EEGlab (Delorme and Makeig, 2004; http://www.sccn.ucsd.edu/eeglab). Scalp map topographies and time courses associated with all ICs were used to identify those components related to stereotyped artefacts which were removed from the data (e.g., eye movements and blinks typically show a far-frontal projection on the map, and are easily spotted by inspecting the time course; muscle artefact components have a typical spatial localization to the temporal sites and show high power at the high frequencies). This was done individually for all participants (on average 7.8 components per dataset, corresponding to $31 \%$ of components).

The remaining, task-relevant components were back-projected onto the original ERP data space and were averaged across trials, separately for each condition. In the back-projected ERPs, epochs were divided in two time ranges: one time interval was time-locked to the onset of the scene (preceding the 'bump' event; 200 to $1000 \mathrm{~ms}$ after onset of the scene), and one was time-locked to the 'bump' event (-200 to $800 \mathrm{~ms}$ after the 'bump' event, or 1320 to $2320 \mathrm{~ms}$ post scene onset; see also Figure 2.1). Only the 'bump' event was considered in the further analysis, because it is a visual event to which the data can be time-locked (such an event is absent in the 'to fly towards' trials). Most importantly, from this time on, it was definite which of the two action verbs applied ('to bump into' or 'to fly towards'). Prior to this point, the speaker could still doubt on which of the two events were to be described, and hence he or she could not anticipate and prepare a description of the event (chance level). Note that in the 'bump' epoch, less trials were included (only the ones in which the figures bumped and not the ones in which the figures flew towards each other, as the visual stimulation differed between these), corresponding to on average 46 trials in ' $S$ ', 47 in 'NP' and 49 in ' $W$ '. For three participants, information on the scene types was not available, hence the analyses on the 'bump' epoch were performed on the remaining thirty participants. The 'bump' epochs were baseline corrected (-200 to $0 \mathrm{~ms}$ after the 'bump' event). 
Based on visual inspection of the grand averages (averaged across all participants), target ERP components and corresponding time windows were specified. Time windows were chosen around the component's maxima (i.e., either in a standard way [peak latency plus and minus $30 \mathrm{~ms}$ for instance] or especially for later, more variable, components - relying on the data itself to choose the most appropriate range) and were kept constant across conditions. For each ERP component, the mean signal per condition and participant was computed.

Statistics on ERP data were performed on the mean amplitude data (computed per time window, per condition, and per participant). We used a repeated measures General Linear Model (GLM) with syntactic complexity as withinsubjects factor (3 levels: W, NP, S) together with two topographical factors: laterality (left, central, right) and anterior-posterior (F, FC, C, CP, P, O) (i.e., in the omnibus tests, a combined total of 18 electrodes were included). Main effects and interaction effects were inspected. Based on interactions between topographical factors and condition, additional analyses were performed on subsets of electrodes. In case of main effects, linear contrasts were inspected first. In case the linear contrasts did not describe the data well, pair-wise comparisons were inspected. Corrections for multiple testing (Bonferroni, in case of pair-wise comparisons) and for sphericity violations (Greenhouse Geisser) were made when necessary. Extreme outlier values ( $>3 *$ interquartile range) were excluded from the analysis. An alpha of 0.05 (corrected) was used as significance level.

\section{Results}

\section{Behavioural data}

\section{Accuracy}

The number of errors varied linearly with syntactic complexity: the more complex the syntax, the higher the number of errors (linear contrast: $F_{1,32}=$ 7.42, $p=.010$; main effect of syntactic complexity: $F_{1.7,53.0}=3.83, p=.035$; 'W': mean $1.09 \%$, SE .23\%; 'NP': mean $1.29 \%$, SE .25\%; 'S': mean $1.81 \%$, SE $.38 \%$ ). The same effect was observed for the amount of corrections (linear contrast: $\left.F_{1,32}=19.56, p<.001\right)$; main effect of syntactic complexity: $F_{1.8,58.8}=$ 14.00, $p<001$; 'W': mean 3.50\%, SE .38\%; 'NP': mean 5.86\%, SE .69\%; 'S': mean $6.48 \%$, SE .68\%; see Figure 2.2 ). 
Inspecting any potential differences in accuracy measures between the two action verbs revealed no main effects of action verb, nor any interaction effects (errors: main effect of action verb $p=.116$, interaction effect $p=.351$; corrections: main effect of action verb $p=.276$, interaction effect $p=.157$ ).
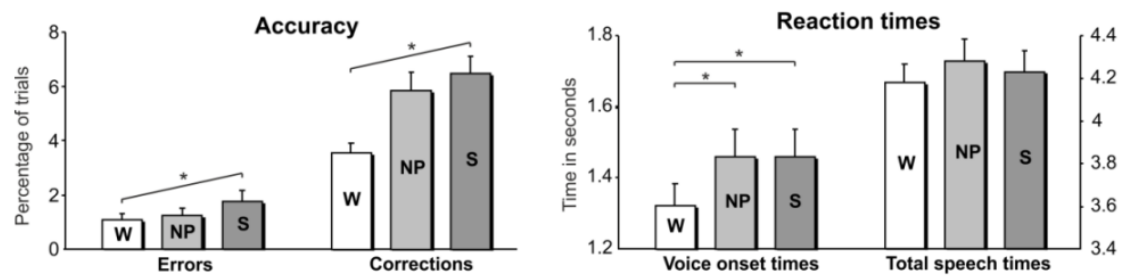

Figure 2.2. Behavioural data. Mean accuracy (left panel) and reaction times (right panel) per condition. Reaction times (plus standard errors) are displayed for voice onset times (VOT; left axis) and for total speech times (TST; right axis). Asterisks indicate significant linear trends (observed for accuracy) or significant contrasts (observed for VOT). 'W' = minimal syntax, word condition, 'NP' = noun phrase-level syntax condition, ' $S$ ' = sentence-level syntax condition.

\section{Reaction times}

The voice onset times (VOT) revealed a main effect of syntactic complexity $\left(\mathrm{F}_{1.5}\right.$, $40.8=20.00, p<.001)$. Contrast analyses showed that in the ' $W$ ' condition the latencies were significantly shorter compared to both the 'NP' and ' $S$ ' condition $(P<.001$, for both cases; 'W': mean $1.30 \mathrm{~s}$, SE .061 s; 'NP': mean $1.43 \mathrm{~s}$, SE $.073 \mathrm{~s}$; ' $\mathrm{S}$ ': mean $1.43 \mathrm{~s}$, SE $.073 \mathrm{~s}$ ). Analysis of the total speech time (TST) revealed a main effect of syntactic complexity $\left(F_{1.5,42.5}=4.65, p=.023\right.$; ' $\mathrm{W}$ ': mean 4.21 s, SE .083 s; 'NP': mean 4.32 s, SE .098 s; 'S': mean 4.28 s, SE .092 $\mathrm{s})$, but the contrast analysis failed to find any significant differences (Figure 2.2).

Analysis of the action verbs revealed no main effect and no interaction effect for the VOTs (main effect $p=.085$, interaction effect $p=.884$ ). However, there was an interaction between action verb and condition in the TST $(p=.017)$. Follow up analysis showed that the 'to bump into' trials resulted in higher TST in all conditions (all $p<.001$; on average $4.50 \mathrm{~s}$ versus $3.96 \mathrm{~s}$ ). Further, only in the 'to fly towards' trials, there was a condition effect $(p=.010)$ : TST was highest for the 'NP' condition. 'NP' differed significantly from 'W' $(p$-corrected $=0.03)$ and marginally significant from ' $S$ ' ( $p$-corrected $=.069$ ). 


\section{ERP data}

Visual inspection of the grand averages showed a clear ERP morphology during the first $1000 \mathrm{~ms}$ post scene onset, followed by a relatively steady period (in which no event-related activity was visible) (see Figure 2.3). Another subset of ERP components was observable at a relatively late time interval (from approximately $1500 \mathrm{~ms}$ after scene onset onwards), in correspondence of the 'bump' event when the target verb was disambiguated (i.e., 'to bump into' instead of 'to fly towards'). The statistical analysis was focused on these two epochs of interest: the first ranged from -200 to $1000 \mathrm{~ms}$ after the scene onset and prior to the 'bump' event (before the action format and thus the verb was available) and the second was related to the time window between -200 to 800 ms after the 'bump' event (when the verb was available, corresponding to 1320 to 2320 ms after scene onset, limited to the 'bump' trials). Statistics were carried out across several time windows. Components belonging to the -200 to $1000 \mathrm{~ms}$ post scene onset time window were labelled as 'scene' components. These were the P1 scene (90-150 ms), the N1/P2 scene (100-240 ms), the P3 scene (350-550 ms), and the fronto-central negativity, post scene $(600-900 \mathrm{~ms})$. The components following the 'bump' event were defined as 'bump' components, namely the P1/N1 bump (20-150 ms), P2 bump (140-280 ms) and the P3 bump (300-500 ms) (Figure 2.3). Note that the labels P1, N1, P2 and P3 are used for descriptive purposes. P1 refers, for instance, to the first positive voltage inflection, $\mathrm{N} 1$ to the first negative voltage inflection and so forth.

\section{Time windows of interest post scene onset}

Time window 90 - 150 ms - P1 post scene: A positive deflection was observed in the 90-150 time window with a clear occipital distribution and a peak around 120 $\mathrm{ms}$ post scene onset. Within this time window, no syntactic complexity effects $\left(F_{2.0,63.9}=0.19, p=.830\right)$, nor any condition-related interaction effects $(p>.15)$ were found.

Time window 100 - 240 ms - N1/P2 post scene: In the 100-240 ms time window, a negative-positive complex was observed with two frontally distributed maxima: a (rather small) negative component peaking at $130 \mathrm{~ms}$ post stimulus, followed by a positive component with a maximum around $210 \mathrm{~ms}$ post stimulus onset. The $\mathrm{N} 1$ was analysed in the $100-160 \mathrm{~ms}$ post stimulus onset window, and showed no effects of syntactic complexity $\left(F_{2.0,63.5}=0.05, p=.946\right)$, nor any syntactic complexity-related interaction effects $(p>.19)$. In the P2 time window (180-240 ms post stimulus), also no syntactic complexity effects $\left(F_{1.9,63.4}=1.62\right.$, $p=.208)$ and no interaction effects $(p>.17)$ were found. 


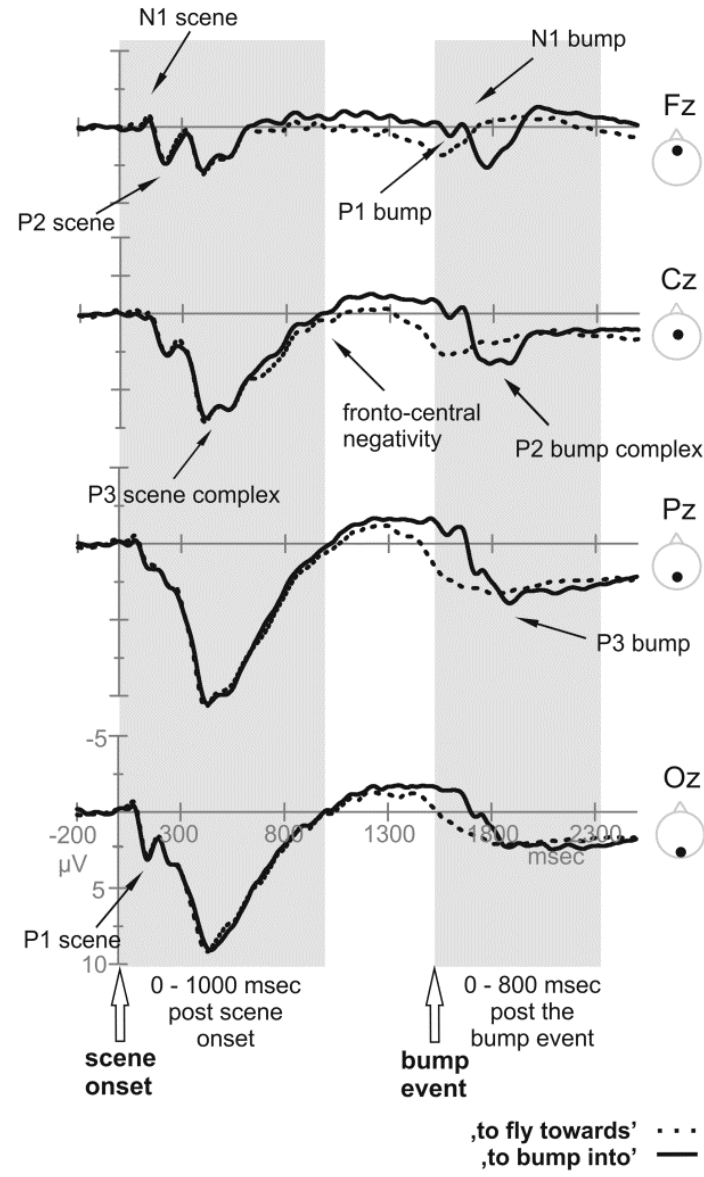

Figure 2.3. Overview of ERPs. Grand average ERPs, separately for the two action formats (solid lines = 'to bump into'; dashed lines = 'to fly towards'), across the midline of the scalp ( $F=$ Frontal, $C$ $=$ Central, $\mathrm{P}=$ Parietal, $\mathrm{O}=$ Occipital) for the entire epoch interval of -200 to $2500 \mathrm{~ms}$ after scene onset, reflecting the speech planning from stimulus onset onwards. The two time windows of interest are highlighted: the post scene onset time window (where scenes of both action formats, and their corresponding ERPs, are still identical) and the post bump event time window (where the analysis was limited to the 'to bump into' trials, as the 'to fly towards' trials did not show an ERP morphology during this time window). Target components are indicated by arrows. Negative voltage is plotted upward in this and all subsequent figures. Note that for plotting purposes, ERP waveforms underwent a low pass filter ( $5 \mathrm{~Hz}, 6 \mathrm{~dB}$ cut-off).

Time window 350 - $550 \mathrm{~ms}$ - P3 post scene: During the 350-550 ms time window, a positivity consisting of a parietal and a more anterior distributed component was observed. The parietal distributed positivity evolved between 350 and $450 \mathrm{~ms}$ with a maximum around $390 \mathrm{~ms}$ after stimulus onset. The 
analysis revealed no syntactic complexity effect $\left(F_{2.0,63.8}=0.08, p=.923\right)$. There was a significant interaction effect between syntactic complexity and the anterior-posterior factor $\left(F_{2.9}, 93.9=4.18, p=.008\right)$, but follow up analyses revealed no significant effects per anterior-posterior plane $(p>.15)$.

The second component was analysed in the time window 450-550 ms post stimulus onset. The overall analysis revealed a trend towards a significant interaction between syntactic complexity and anterior-posterior $\left(F_{2.6,82.9}=2.64\right.$, $p=.063)$. Simple contrasts showed that only at frontal electrodes $(F)$, a significant syntactic complexity effect was present $\left(F_{1.9,63.1}=3.99, p=.025\right)$ (FC: $p=.287 ; \mathrm{C}: p=.719 ; \mathrm{CP}: p=.772 ; \mathrm{P}: p=.976 ; \mathrm{O}: p=.801)$. Pair-wise comparisons at $\mathrm{F}$ showed a significant difference between ' $\mathrm{W}$ ' and ' $\mathrm{NP}$ ' $\left(F_{1,32}=\right.$ 6.31, $p$-corrected $=.017$ ) and a trend towards a difference between ' $\mathrm{W}$ ' and ' $\mathrm{S}$ ' $\left(F_{1,32}=4.00, p\right.$-corrected $\left.=.054\right)($ see Figure $2.4 \mathrm{~A})$.

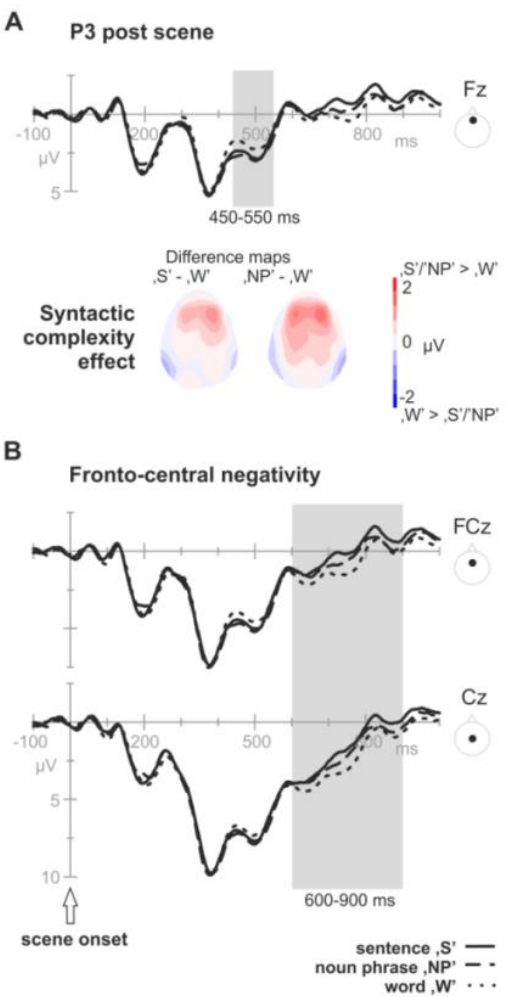

Figure 2.4. Syntactic complexity effects following scene onset. Grand average ERPs, separately for the three syntactic complexity formats ('S', 'NP', 'W'), in the post scene onset epoch. A) The P3 syntactic complexity effect ('S'/'NP' > 'W') at frontal midline electrode (Fz) within the time window 450-550 ms post scene onset, together with the topography maps of the effect distribution across 
the scalp (bottom; left: ' $S$ ' minus ' $W$ '; right: 'NP' minus ' $W$ '). B) The fronto-central negativity that modulated with syntactic complexity (linear effect: 'S' > 'NP' > 'W') at fronto-central and central midline electrodes ( $\mathrm{FCz}, \mathrm{Cz}$ ) within the time window $600-900 \mathrm{~ms}$ post stimulus onset.

Time window 600 - $900 \mathrm{~ms}$ - fronto-central negativity post scene: A negative component was most prominently visible at fronto-central sites in a rather late time window (600-900 ms post scene onset). In the overall analysis, a trend towards an interaction effect was found between syntactic complexity and anterior-posterior $\left(F_{2.6,83.0}=1.83, p=.055\right)$. Simple effect analyses on the fronto-central plane $(\mathrm{FC}, \mathrm{C})$ revealed a significant syntactic complexity effect $\left(F_{2.0,63.2}=3.60, p=.034\right)$ (at other electrode planes, $\left.p>.05\right)$. Contrast analysis confirmed a linear relation $\left(F_{1,32}=6.80, p=.014\right)$ : higher syntactic complexity related to higher negativity of the target amplitude (see Figure 2.4B).

\section{Time windows of interest post bump event}

Time window 20 - $150 \mathrm{~ms}$ - P1/N1 post bump: In the 20-150 ms time window, a positive component was observed (20-80 ms post bump event) followed by a negative component (70-150 ms post bump event), both having a central distribution. No significant syntactic complexity effects were found (P1 component: $F_{1.6,47.3}=2.05, p=.148$; N1 component: $\left(F_{1.8,52.1}=2.93, p=.068\right)$, nor any significant interactions effect in either component (all $p>.1$ ).

Time window 140 - 280 ms - P2 post bump: In this time window, a component complex was visible with an earlier posterior distribution and a later frontocentral topography. Within this $140-280$ ms time window, no significant syntactic complexity effect was found $\left(F_{1.6,47.5}=2.64, p=.091\right)$, nor any condition related interaction effect $(p>.17)$.

Time window 300 - 500 ms - P3 post bump: A positive component was observed in the 300-500 ms time window, having a posterior distribution. In addition to an overall marginally significant syntactic complexity effect $\left(F_{1.8,47.3}=3.29, p=\right.$ .052 ), there was an interaction between syntactic complexity and anteriorposterior distribution $\left(F_{2.0,54.6}=4.76, p=.012\right)$. Simple effect analyses showed that only at posterior sites (CP, P), there was a significant syntactic complexity effect $\left(F_{1.8,47.6}=4.45, p=.021\right)$ (at F, FC: $\left.F_{1.8,48.5}=1.92, p=.161\right)$. Pair-wise comparisons showed that ' $S$ ' significantly differed from 'NP' and differed marginally from ' $\mathrm{W}$ ' ( $p$-corrected $=.004$ and $p$-corrected $=.067$, respectively), where 'S' was more positive than ' $W$ ' and 'NP' (see Figure 2.5). 


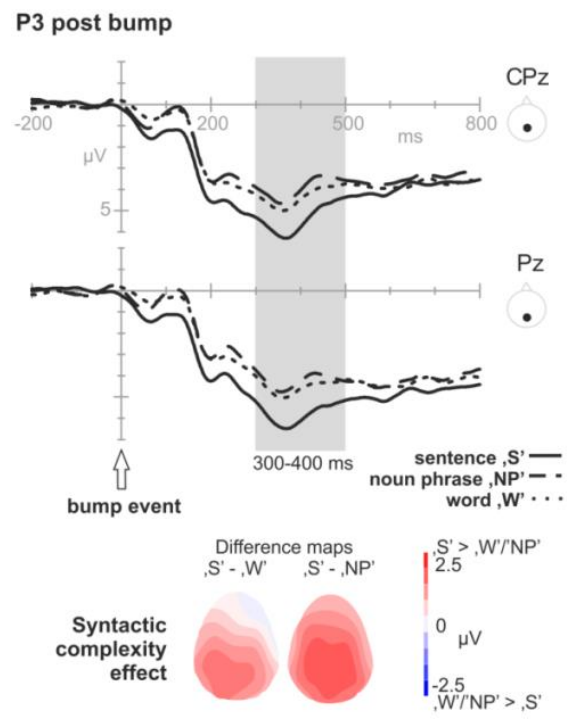

Figure 2.5. Syntactic complexity effects following verb disambiguation: Grand average ERPs, separately for the three syntactic complexity formats ('S', 'NP', 'W'), in the post 'bump' epoch. Top: Signals from the centro-parietal and parietal midline electrodes ( $\mathrm{CPz} P \mathrm{Pz})$. The gray-shaded area indicates the P3 bump syntactic complexity effect ('S' > 'NP'/'W') within the time window 300-500 ms post bump event. Bottom: The P3 effect distribution as a topographic map (left: 'S' minus 'W'; right: ' $S$ ' minus 'NP'). Note that for plotting purposes, ERP waveforms underwent a low pass filter $(5 \mathrm{~Hz}, 6$ dB cut-off).

\section{Discussion}

The aim of the present study was to investigate when syntactic encoding takes place during sentence planning. The sentence planning was triggered by a visual scene of moving objects. We asked participants to overtly describe these scenes using naming formats with parametrically varying syntactic complexity (using single words ' $W$ ', noun phrases 'NP', or a complete sentence 'S'). We assumed that any variation in neural activity related to syntactic complexity would be reflected at the level of the ERP signal. Further, the design of the paradigm allowed us to temporally separate initial noun phrase planning starting at scene onset, from planning at sentence-level (occurring after all information is available, or after target action/verb disambiguation). Based on serial syntactic processing views, we expected ERP modulation around 300-500 ms after scene onset (associated with noun phrase-level syntactic planning). We based this hypothesis on previous behavioural studies on single word productions (Indefrey and Levelt, 2004) and on more recent electrophysiological studies using sentence production paradigms (Marek et al., 2007; Sahin et al., 2009). 
However, we did not exclude the possibility of early and non-additive neural modulations, as might be predicted by connectionist or interactive models in which visual, conceptual and syntactic processes are initiated in parallel and influence each other (e.g., Elman, 1991; Kempen, 2013), or might not even be viewed independently (e.g., Langacker, 1987).

Behaviourally, both the number of errors and corrections showed a linear relation with syntactic complexity (the more syntax, the more errors and corrections), indicating that the intended complexity manipulation was successful. The linearity of the pattern can be interpreted as support for increasing syntactic complexity. Syntactic complexity did not influence the total speech time (TST). The TST, however, differed across action verbs, where 'to bump into' trials resulted in longer TSTs in all conditions, compared to 'to fly towards' trials. As we analysed only the 'to bump into' trials in the post bump time window, this does not pose any difficulties for our results in this window. The type of action verb did not affect any of the other behavioural measures, excluding the possibility of confounding effects in the post scene epoch where both action verbs were analysed together. With respect to the voice onset time (VOT), we found that the word-'W' condition differs from both the 'NP' and ' $S$ ' conditions. In particular, VOT was shorter for the ' $W$ ' condition (on average 1.30 $\mathrm{s}$ in contrast to $1.43 \mathrm{~s}$, for both 'NP' and ' $\mathrm{S}$ '), suggesting that prior to the initiation of the utterance there is already syntactic planning at the level of the noun phrase. Consistent with that, 'NP' and ' $S$ ' both require planning of noun phrases, while ' $W$ ' does not. The encoding requirements in 'NP' and 'S' do not differ at this moment, while they both differ from ' $W$ '. From this data and design, however, we cannot distinguish whether this planning entails syntactic retrieval and morpho-syntactic processing (inflections) or syntactic structure building (assuming the structure is built incrementally), or both. Either explanation would fit the observed modulation. Nevertheless, the data confirm the idea that a speaker plans in advance (Bock, 1995; Meyer, 1996; Wagner et al., 2010), in this case including already noun phrase related syntactic encoding. This is in line with previous work on the production of noun phrases (Alario et al., 2002; Schiller and Caramazza, 2006). It should be noted, however, that both the extent and the nature of the advance planning might have been imposed by the design, because it poses constraints on the available information at this time. Previous studies have shown that the extensiveness of utterance planning can be varied depending on the speakers' experience (in this case repetition of utterances), the circumstances (in this case availability of information), and cognitive abilities (see e.g., Konopka, 2012; Wagner et al., 2010). 
Compared to previous work on production of multi-word utterances, the VOTs are relatively long. In a Dutch noun phrase production study, VOTs varied around 580-670 ms, depending on the condition (picture word interference paradigm) (Schiller and Caramazza, 2006). In another study in which participants produced noun phrases, VOTs were around 660-720 ms (Alario et al., 2002). However, an important difference between the design used in those experiments and in the present one, is the stimulation. In the current study, participants were instructed to describe a moving scene. The intended message, therefore, has to be derived from a scene consisting of several frames. In animations, as opposed to static pictures, it is not immediately clear from the start position which figures are going to be involved in the action. The information thus has to be integrated over time, which can explain the elongation of VOTs. In addition, it is important to note that the VOTs we observed are quite similar to the reaction times reported by Indefrey et al. (2001), which used similar stimulation (1.29 $\mathrm{s}$ for ' $\mathrm{S}$ ', 1.28 for 'NP' and 1.23 for 'W'; reflecting similar VOTs and effects).

The observed ERPs related to overt speech production had a similar morphology for all conditions ('W','NP' and 'S'), with a clearly visible P1, N1, P2, P3 complex and a fronto-central negativity following scene onset. Another P1, N1, P2 and P3 morphology were found after the 'bump' event (when all information including the verb was available). The first divergence across syntactic complexity conditions started approximately from 400 ms post scene onset on. We will discuss the components and their potential syntactic complexity modulations in chronological order, starting from the moment of scene onset.

\section{Initial syntactic planning}

Early components: The first components after scene onset - P1 and N1/P2 showed no variations across syntactic complexity conditions, indicating similar demands on the early processing functions. The early components have been associated with early perceptual processes, with attention (P1 and N1, Hillyard et al., 1998), with the early (pre)verbal stages of conceptual knowledge activation (linked to the P1, Rabovsky et al., 2012; Rahman and Sommer, 2008) and with lexical access (P2 related, Bles et al., 2007; Costa et al., 2009; Strijkers et al., 2009). Although it can never be ruled out completely that the response instructions resulted in differential preparatory states perceptual or conceptual processing (see e.g., Price and Friston, 1997), the finding that these early ERP components were not modulated by our manipulation, indicates that 
we succeeded to keep the variance of visual and conceptual processing to a minimum over the three utterance conditions.

P3 scene: In a specific window, between 350 and 550 ms post scene onset, a P3-like component was clearly visible. It comprised two subcomponents: one with a posterior scalp distribution and one with a more anterior focus. No variation with syntactic complexity was found in the posteriorly distributed activity (350-450 ms post scene onset). At anterior sites variation with syntactic complexity was present within the P3 time window (450-550 ms). The word-'W' condition significantly differed from the noun phrase-'NP' and (marginally) from the sentence-' $S$ ' conditions, where 'NP' and ' $S$ ' elicited a higher positivity compared to ' $W$ '. At this time point, the action format of the scene (verb) was still ambiguous, but visual input was sufficient to give way to first noun phrase planning (nouns and adjectives). 'W' did not require retrieval of syntactic information or inflections at a noun phrase level, while the noun phrase 'NP' and sentence ' $S$ ' condition did (e.g., the retrieval of the syntactic gender reflected in the adjective [in Dutch 'de/het] and the inflection of the adjectives ['groen > groene; green]). The noun phrase-related syntactic processing might in turn be reflected in higher $\mathrm{P} 3$ amplitudes at frontal sites.

Syntax-first language accounts assume that utterance structure is build prior to any lemma retrieval and morpho-syntactic processing (Friederici, 2011). The observed data would also support such a view. Either this component reflects incremental structure building of the noun phrases, or it might be that the structure is already be available, and the modulation reflects online filling of information into the structure. Processing requirements for 'NP' and ' $S$ ' do not differ for both scenarios, but they both differ from 'W'. Most importantly, the observed ERP modulation between 450-550 ms post scene onset indicates that this time window is sensitive to syntactic noun phrase planning.

In a previous electrophysiological production study, the $\mathrm{P} 3$ has been associated with conceptual and/or syntactic complexity (350-500 ms post stimulus onset, Marek et al., 2007), but the distribution of this component was centro-parietal (while in the current study, the effect was anterior). It seems unlikely, however, that the present effect reflects conceptual planning, as previous studies found conceptual effects in earlier time windows (e.g., $120 \mathrm{~ms}$ post stimulus presentation, Rahman and Sommer, 2008), and we did not find such early modulations. Also, the design of the current study minimized conceptual processing. Further, with respect to timing, the result is in line with studies on the time course of single word production of Indefrey and Levelt (2004), with Koester and Schiller's study on morphological encoding (priming effects were found 350-650 ms after picture onset, 2008) and with Sahin et al.'s study (2009) 
who suggested that syntactic encoding starts around $320 \mathrm{~ms}$ post stimulus onset (although paradigms differ). In these previous studies, syntactic structure building was not required, suggesting that the $\mathrm{P} 3$ effect observed in the current study does not reflect structure building only. In more general, non-linguistic terms, the P3 has been associated with a monitoring function, context updating and working memory actions (see e.g., Coles and Rugg, 1995). It has also been proposed that the $\mathrm{P} 3$ amplitude reflects activities in a network controlled by joint operations of both attention and working memory (Kok, 2001). Whether the ERP effect observed in this study reflects directly the differential demands on syntactic or differential demands on attention and working memory processes accompanying the linguistic processes cannot be disentangled. The present P3 result shows that syntactic modulation either directly (direct modulation of the P3) or indirectly (P3 modulation via attention and processing load) correlates with neural activation in this time window, indicating active syntactic processing in this time range.

Late negativity: The data also revealed a clear linear relation of syntactic complexity across naming conditions within a (bilateral) fronto-central negativity at $600-900 \mathrm{~ms}$ post scene onset. In particular, we observed that - in terms of amplitudes - 'S' elicited the most negative activity, followed by 'NP' and ' $W$ '. To our knowledge there is no previous report on such an ERP modulation during overt sentence production planning. We can only speculate about its interpretation here based on the complexity manipulation in our experiment. This fronto-central negativity might reflect directly (continued) syntactic structure building of the sentence to be uttered, as the syntactic structures varied across the three conditions, and can be anticipated on. Some language accounts, however, suggest that structure building already occurs relatively early in sentence production (Konopka, 2012), which would not be in agreement with the observed rather late ERP modulation. Alternatively, it might reflect modulated working memory demands or a check/control monitoring on the appropriateness of the planning so far.

\section{Sentence-level planning}

Early post bump components: After a period of activity around baseline, without any clear distinguishable ERP components (from approximately 1000-1400 ms post scene onset), another temporal event occurred in the visual stimulation: the 'bump' event. At that moment in time, it became definite which of the verbs had to be used ('to bump into' or 'to fly towards'). In the 'bump' trials - time-locked to the clearly defined 'bump' event - another set of ERP components arose that were absent in the 'to fly towards' trials. This absence was most likely due to the 
lack of a clear temporal event in the latter condition. We assume that similar cognitive processes occur in these 'to fly towards' trials, but they do not occur in temporal synchrony to an external event - as there is no such event. Hence, they cannot be detected by the averaging model of ERPs. The ERPs related to the 'bump' event showed a centrally distributed $\mathrm{P} 1 / \mathrm{N} 1$ and a subsequent P2 complex (comprised of a posterior and more fronto-central component), but no syntactic complexity effects in these component. Analogous to the early post scene onset ERP components, these ERP components are most likely associated with more perceptual, conceptual, and basic attention processes related to the 'bump' event. Their insensitivity to the complexity modulation suggests again comparable visual and conceptual processing across conditions. Note that around the time of this post 'bump' epoch, the voice onset started on average (1.3 - $1.4 \mathrm{~s}$ after stimulus onset), which has been reported in the past to cause high frequency artefacts in the ERP signal (see also Ganushchak et al., 2011). To avoid noise in the data caused by artefacts, we used ICA to clean the data. Independent components related to eye and muscle artefacts were filtered out of the data. As a result, we were able to observe a clear ERP morphology. Note that this is of interest from a methodological point of view, as the applied pre-processing revealed interpretable production ERPs within overt naming trials, even in relatively late time windows.

P3 post bump: We again observed variation with syntactic complexity within the P3-time window, but with a different, more parietal distribution (instead of a frontal distribution). In addition to differences in topographic distribution, we observed a difference in amplitude modulations across conditions. The ' $S$ ' condition was significantly more positive compared to 'NP' and marginally compared to ' $W$ ' ('NP' and 'W' did not differ from each other). The pattern of the complexity effect thus differed from the post scene P3 (where ' $S$ ' and 'NP' were more positive compared to ' $W$ '), which is an interesting functional segregation of two types of P3 effects. The difference in topography further suggests two different sources for the post scene onset P3 and post bump onset P3. At this moment in time (300-500 ms post bump event; or 1820-2020 ms post scene onset), all information was available to the participant (including the type of verb). Under the assumption that planning of the first noun phrases was already initiated immediately after scene onset, it is likely that planning within this later time window was related to local encoding of the newly available element - the verb - (e.g., lexical access, inflection) and to the (potentially continued) assembly of the utterance in general. The specific pattern of syntactic complexity effects is consistent with the idea that only in ' $S$ ' inflection of the verb plus assembly of all elements into a syntactically well-formed utterance was needed, while in 'NP' and ' $W$ ' this was not necessary. In the latter cases, the 
verb was expressed as its unmarked form (infinitive) and the word order was according to a predefined format.

Taken together, the complexity modulation in this study was reflected in both modulations in behaviour and in the ERP. In the fronto-central negativity we observed a linear pattern (the more syntactic planning, the higher the amplitude). In both the P3 following scene onset and following verb disambiguation, we observed a different complexity variation. This variation was segregated in terms of function and topography (amplitude modulations and distribution differed, respectively), indicating different neural sources. The pattern in the components suggests that the frontal P3 reflects early noun phrase planning, while the later parietal P3 indicates noun phrase assembly and integration processes. While other studies have already observed syntactic modulations in the (first) P3 time window (e.g., Marek et al., 2007; Sahin et al., 2009), the current study is the first to delineate syntactic sentence planning over time and to investigate the entire time window, using a more realistic display of moving objects instead of static line drawings of scenes. Therewith, it extends the findings of previous studies and demonstrates the possibility of investigating relatively late components of sentence production in a naturalistic manner.

The observation of clear, distinguishable - relatively late - time windows sensitive to syntactic encoding and a lack of any early ERP effects, seems not to support integrative accounts that assume early initiation of all processes. However, it cannot be excluded that the observed stage-like behaviour in the data emerged as a property of parallel accounts (Kempen, 2013). Speculatively, the data do not seem to directly support evidence for language as an emergent property, as one might expect enhanced planning for the new, un-learned utterance structures ('W', and in lesser extent 'NP') compared to the natural and learned structure of the sentences in 'S'. However, other experimental setups would be needed to test such models directly and explicitly.

The results are in agreement with incremental encoding of the utterance, unfolding over time. From the current design, however, we cannot distinguish whether lemma retrieval/assignment and morpho-syntactic processing comes first, or only after syntactic structure building.

Although linear effects were expected, based on the parametric variation across conditions and the results of the PET study (Indefrey et al., 2001; 2004), this was not always the case. The effect in both P3 components was not linear, but reflected a different modulation ('W' versus 'NP'/'S', and 'W'/'NP' versus 'S', respectively). Notably, the current ERP study was able to delineate the timeinsensitive PET result over time. By exploiting the high temporal resolution and certain aspects of the design, the current ERP results give us more insight in the 
temporal aspect of syntactic encoding. The observed ERP pattern further suggests that the overall PET effect is a summation of neural activity within different time windows and with distributed neural sources. The functional role of the left IFG and the observed linear correlation with syntactic complexity has to be re-evaluated in future experiments.

Inherent to the study of syntactic encoding is that it is impossible to create a pure manipulation of syntactic planning, as it never occurs in isolation and is difficult to manipulate without changing any of the other processes (Ganushchak et al., 2011). For instance, we cannot exclude differential perceptual effects across conditions caused by the instructions, as certain naming format in a given block may alter the perception and degree of attention to certain objects. The observed lack of effects in the early ERP components supports the idea that any perceptual and attentional differences were negligible in the present design.

In addition, all three conditions required temporal ordering of the words into an utterance. This temporal ordering is related to conceptual encoding (Levelt, 1989) and might involve some form of structure building. Next to the need of listing adjectives and nouns in a serial order, the infinite verb in 'W' and 'NP' is also a phrase ("naar toe vliegen" or "to fly towards"). Overall, ordering and minimal structure encoding might have decreased the net difference between complexity conditions. However, the lack of early ERP effects and the later, observed complexity effects, indicate that the conceptual ordering was not different across conditions, and that the complexity manipulation was sufficient to be reflected in the data, respectively.

Another limitation of the study was that the blocked design resulted in repetitions of the same response condition, and thus the same type and structure. Repeating the same structure across trials and conditions was chosen to keep conceptual processes as constant as possible, but may have potentially caused priming effects. It is plausible that structural priming effects might have occurred in the current study, facilitating the processing of a subsequent utterance with the same structure (Pickering and Branigan, 1999). The priming might have reduced planning of the structure across trials. Nevertheless, even in face of potential structural priming effects, the syntactic complexity modulation was robustly found in several components. In future research, the use of filler trials requiring different utterance structures could be considered, to avoid structural priming effects and potentially increase the magnitude of the effects. 


\section{Conclusion}

In this study, we have examined the temporal aspects of syntactic encoding in sentence production. ERPs were cleaned from (muscle) artefacts using ICA, and we observed a clear ERP morphology. Event related potentials associated with immediate noun phrase-planning were found starting from scene onset on. By exploiting the fact that verb availability was not immediate, but temporally defined by an event, we were able to investigate relatively late ERPs related to noun phrase assemblies and overall sentence integration. More specifically, we found that overt description of a movie-like scene elicited very similar P1/N1/P2 components across all complexity conditions (words, noun phrases, or sentence format). From $400 \mathrm{~ms}$ onwards, conditions started to deviate in specific time windows. In particular, we found three components showing a modulation with syntactic complexity: following scene onset an anterior P3 scene effect (at 450550 ms post scene onset; 'S'/'NP' > 'W') and a fronto-central negativity (at 600900 ms post scene onset; 'S' > 'NP' > 'W') were observed, and following the 'bump' event another, more posterior, P3 effect (300-500 ms after verb availability; 'S' > 'NP'/'W'). We interpret the components in the first time window - the P3 scene and fronto-central negativity - as related to syntactic encoding of noun phrases. The P3 related syntactic encoding here seems to involve the retrieval of syntactic information, such as inflections, and the assembly of words into phrases, in which 'S'/'NP' differ from 'W'. The late negativity seems sensitive to syntactic structure building as it modulates differently across the three conditions. The ERP component in the later time window - the P3 bump is related to more global syntactic planning at the sentence level. This may involve encoding of the verb and continued assembly of the utterance, in which 'S' differs from 'NP'/'W'. The data show that the P300 time window is sensitive to syntactic planning, both at noun phrase-level and at sentence-level. The functional segregation and differential topographical distributions of the P3 components further indicates different neural sources, suggesting that noun phrase planning and sentence-level planning require different cognitive operations.

\section{Acknowledgments}

We kindly acknowledge Anke Sambeth and Petra Vlamings for training and support with the EEG equipment, recordings and analyses. The project was funded by a Maastricht University incentive (Mobilizing Minds: "Females into higher positions" to BM Jansma). 



$$
\sim
$$


CHAPTER 3

\section{FROM MIND TO MOUTH: EVENT RELATED POTENTIALS OF SENTENCE PRODUCTION IN CLASSIC GALACTOSEMIA}

Inge Timmers, Bernadette M Jansma, and M Estela Rubio-Gozalbo (2012). From Mind to Mouth: Event Related Potentials of Sentence Production in Classic Galactosemia. PLoS ONE. 7(12): e52826. 


\section{Summary}

Patients with classic galactosemia, an inborn error of metabolism, have speech and language production impairments. Past research primarily focused on speech (motor) problems, but these cannot solely explain the language impairments. Which specific deficits contribute to the impairments in language production is not yet known. Deficits in semantic and syntactic planning are plausible and require further investigation. In the present study, we examined syntactic encoding while patients and matched controls overtly described scenes of moving objects using either separate words (minimal syntactic planning) or sentences (sentence-level syntactic planning). The design of the paradigm also allowed tapping into local noun phrase- and more global sentence-level syntactic planning. Simultaneously, we recorded event-related potentials (ERPs). The patients needed more time to prepare and finish the utterances and made more errors. The patient ERPs had a very similar morphology to that of healthy controls, indicating overall comparable neural processing. Most importantly, the ERPs diverged from those of controls in several functionally informative time windows, ranging from very early (90-150 ms post scene onset) to relatively late ( $1820-2020 \mathrm{~ms}$ post scene onset). These time windows can be associated with different linguistic encoding stages. The ERP results present the first neuroscientific evidence for language production impairments in patients with galactosemia in lexical and syntactic planning stages, i.e., prior to the linguistic output phase. Hence, these findings shed new light on the language impairments in this disease. 


\section{Introduction}

Patients with classic galactosemia, an inborn error of galactose metabolism, have speech and language production impairments whereas comprehension is relatively preserved (Potter et al., 2008; Waisbren et al., 1983). Such impairments can be burdensome to patients as they might hamper communication and hence social interactions. Nevertheless, underlying language processing components and neural correlates of these impairments are poorly understood.

In classic galactosemia, there is a deficiency of the enzyme activity that converts galactose-1-phosphate (gal-1-P) into UDP-galactose (i.e., the galactose-1-phophate uridyl transferase [GALT] enzyme). This is due to mutations in the GALT gene, located on the short arm of chromosome 9. A galactose-restricted diet resolves the neonatal toxic symptoms, but cannot prevent the emergence of cognitive difficulties such as lowered intelligence, memory problems, slower general information processing and impaired speech and language production (Antshel et al., 2004; Doyle et al., 2010; Potter et al., 2008; Potter, 2011; Schadewaldt et al., 2010; Schweitzer et al., 1993; Waggoner et al., 1990; Waisbren et al., 2012; Widhalm et al., 2002), while receptive language or comprehension is relatively preserved (Potter et al., 2008). Voice and motor speech disorders (e.g., childhood apraxia of speech or dysarthria) have been reported (Nelson et al., 1991; Potter, 2011; Robertson et al., 2000; Shriberg et al., 2011; Webb et al., 2003) as well as problems with word retrieval, grammar and vocabulary (the latter impairments are related to the planning of a message and not with the verbal output of a message) (Schweitzer et al., 1993; Waggoner et al., 1990; Waisbren et al., 1983). Although patients can experience a broad spectrum of cognitive impairments, the speech and language impairments cannot be solely explained by lower cognitive abilities in general (Potter et al., 2008; Waggoner et al., 1990; Waisbren et al., 1983) (e.g., some patients with low intelligence have no language impairments, while others with average intelligence have language impairments, Potter et al., 2008). Hitherto, the main focus of research, diagnosis and treatment has been on speech (output) difficulties (e.g., on voice disorders or childhood apraxia of speech (Nelson et al., 1991; Potter, 2011; Robertson et al., 2000; Shriberg et al., 2011). However, speech (output) impairments cannot solely explain the language impairments. Language production is a complex process comprising multiple processing stages prior to the output stage (Bock, 1995; Indefrey, 2011; Levelt, 1989). In galactosemia, nonetheless, it has never been studied how language production is affected. In this study, we took a cognitive point of view, examining language production using psycholinguistic 
models. In the remainder of this manuscript we will refer to 'language production' as specified in the field of linguistics and cognitive neuroscience, namely describing the cognitive phases that are involved in planning a message prior to the articulation.

Psycholinguistic models of language production suggest cognitive stages in which relevant language information is planned over time. First, an intended message has to be transferred into a conceptual/semantic representation. Appropriate lexical entries are selected and retrieved as well as the corresponding grammatical and syntactic information. Structural syntactic frames are constructed and assembled or filled in producing a well-formed utterance. Finally, the message is encoded and articulated (Bock and Levelt, 1994; Bock, 1995). The language production process has been investigated in many picture naming experiments using reaction times (see Levelt et al., 1999, for a review) and event related potentials (ERPs, derivatives of the electroencephalogram, Kutas and Schmitt, 2003). This way, sensitive time windows have been suggested for the language production stages. It has been shown that conceptual information is activated around $120 \mathrm{~ms}$ after stimulus onset (e.g., Rahman and Sommer, 2008), followed by semantic processing. This is followed by syntactic encoding approximately $90 \mathrm{~ms}$ later, serving as input to phonological encoding after another $40 \mathrm{~ms}$. The processes are not fully serial but might overlap in time, suggesting cascading information flow over time (see for an overview Indefrey, 2011; Jansma et al., 2004; Schmitt et al., 2000; van Turennout et al., 1997). Each of the planning steps can be linked to specific brain areas within a cortical network (see overviews in Hickok, 2009; Indefrey, 2011; Price, 2010). Ignoring other potentially relevant factors for a moment, any type of impairment might therefore be directly related to dysfunction within this network. Lesions within specific areas may affect production and comprehension separately (Friederici, 2006; Rogalski et al., 2011), whereas disruptions of connectivity between areas may delay or disturb language processing (Glasser and Rilling, 2008). Few imaging studies have been conducted in galactosemia, observing anatomical brain abnormalities, such as white matter abnormalities, cerebral and cerebellar atrophy (Kaufman et al., 1995; Nelson et al., 1992), but it remains uncertain whether specific areas or networks might be particularly affected.

A screening of our patient cohort's medical files suggested a syntactic deficit in the patients with galactosemia as their utterances were described as short, simple and frequently as syntactically incorrect. Necessary steps in syntactic encoding are identification and activation of grammatical information associated with the concepts (e.g., whether it is a noun or adjective; lexical selection), the 
assignment of syntactic relations or grammatical functions to each word (e.g., subject versus object; function assignment), inflection of words (e.g., $-s$ for plural, -ed for past tense) and assembly of words into so called syntactic structural frames, i.e., syntactic plans (constituent assembly) (Bock and Levelt, 1994; Bock, 1995). It deduces that in syntactic planning more local phrasal-level planning (first steps described) can be distinguished from more global sentencelevel planning (assembly into a frame and utterance). Especially in multi-word utterances, it is believed that the scope of planning is incremental such that the utterance can be initiated as soon as certain elements are available (e.g., Allum and Wheeldon, 2007; Allum and Wheeldon, 2009; Bock, 1995; Levelt, 1989). The amount of advance planning is suggested to be in terms of functional phrases (e.g., Allum and Wheeldon, 2009), but is also dependent on the cognitive load of the utterance and the cognitive capacity of the speaker (Bock, 1995; Meyer, 1996; Wagner et al., 2010). In healthy controls, syntactic processing has been studied in the context of syntactic anomalies or syntactic complexity during comprehension (P600 and left anterior negativity [LAN] ERP components) (e.g., Hagoort, 2003; Kaan et al., 2000). In terms of brain areas, syntactic encoding and sentence processing have been related to the left inferior frontal gyrus (i.e., left IFG, encompassing Broca's area, Hickok, 2009; Indefrey et al., 2001; Indefrey et al., 2004; Sahin et al., 2009). In comprehension research, studies assume that the left IFG is retrieving and integrating lexical information from long term memory, most likely from left temporal areas (Hagoort, 2003; Hagoort, 2005; Snijders et al., 2009; Vosse and Kempen, 2000). A similar process can be assumed for speech planning in which concepts have to be integrated into proper syntactic and phonological frames (see Sahin et al., 2009, for first empirical indications using intracranial electrophysiology).

In the present study, we aimed to investigate whether patients with classic galactosemia have impairments in sentence production by recording high temporal resolution ERPs during a language task. This method allowed us to track the neural activity related to the entire language planning process from the intention to speak onwards, across sensitive time windows. Comparing the patients' ERP (i.e., morphology of the wave, amplitude and latency of components) with that of healthy controls gives us an indication on whether syntactic encoding is intact, delayed, or malfunctioning at a millisecond time resolution. An experimental paradigm was used that elicits overt utterances in response to an animated scene in a relatively natural manner. Through different instructions, the reports of the scene varied in syntactic complexity (Indefrey et al., 2001; 2004), allowing us to study syntactic effects within the ERP. The content of the scenes differed from trial to trial (i.e., the geometrical figure, colour of the figures and verb) and not all information was available from the 
scene onset (i.e., the verb; the actor could either 'bump into' or 'fly towards' the other figure; both scene variations start visually identical, and diverge at a certain point). The participants therefore could not anticipate the action of the figure, ensuring active generation of the utterances (instead of only automated processes). Further, it allowed us to tap into both early local phrasal-level planning of noun phrases (starting immediately after scene onset, associated with initiation of planning the first elements of the utterance that are already available: the first nouns and corresponding adjectives) and on later global sentence-level planning (when all relevant information is at hand, including the verb; adding the construction of the utterance). Time windows of any deviations, relative to the visual stimulation, give information on whether differences are related to early conceptual, early local syntactic, or rather late global syntactic or articulatory processing. Specifically, variation with syntactic complexity would reflect time windows relevant for syntactic encoding during sentence production. Moreover, relevant cognitive functions (i.e., visual memory, attention, working memory) were studied independently using standardized tests and related to the ERP data in order to exclude possible confounding of these more basic functions.

\section{Materials and methods}

\section{Ethics statement}

The Medical Ethical Committee of Maastricht University Hospital/Maastricht University (azM/UM) gave ethical clearance for this study. All participants, and for minors also both parents/caregivers, gave written informed consent.

\section{Participants}

Twenty-four adolescent patients with classic galactosemia and twenty-one healthy controls participated in this study. Classic galactosemia was diagnosed by GALT enzyme activity assay and/or GALT-gene mutation analysis. Two participants (both patients) were excluded because of difficulties executing the ERP task. Patient characteristics can be found in Table 3.1. Of the remaining 22 patients, 15 were female and 7 male, mean age 14.9 years (SD 2.2 years, range 10.8 - 19.1 years). The control group consisted of 14 females and 7 males mean age 14.2 years (SD 1.8 years, range 11.4 - 17.0 years). Neither gender nor age differed significantly between the groups $\left[F_{1,41}=.01, p=.92\right.$ and $F_{1,41}=1.07, p$ $=.31$, respectively]. Participants had no other relevant health condition, all had normal or corrected to normal vision, and were native Dutch speakers. 
Table 3.1. Galactosemia patient characteristics

\begin{tabular}{|c|c|c|c|c|}
\hline & $\mathbf{N}$ & Mean & SD & $\begin{array}{l}\text { Range of } \\
\text { values }\end{array}$ \\
\hline Age at diagnosis (in days) & 22 & 12.4 & 14.3 & $0-60$ \\
\hline $\begin{array}{l}\text { Age at introduction of diet } \\
\text { (in days) }\end{array}$ & 22 & 12.2 & 14.4 & $0-60$ \\
\hline $\begin{array}{l}\text { GALT activity (in \% of mean } \\
\text { reference value) }\end{array}$ & 20 & 0.60 & 0.57 & $N D^{3}-1.83$ \\
\hline $\begin{array}{r}\text { Urine galactose level } \\
\text { (in } \mu \mathrm{mol} / \mathrm{mmol} \text { creatinine) }{ }^{4}\end{array}$ & 22 & 12.0 & 21.1 & $N D^{3}-96$ \\
\hline $\begin{array}{r}\text { Urine galactitol level } \\
\text { (in } \mu \mathrm{mol} / \mathrm{mmol} \text { creatinine) }{ }^{4}\end{array}$ & 22 & 132.0 & 22.8 & $94-187$ \\
\hline Special education ${ }^{5}$ & 22 & $68.2 \%$ & & \\
\hline Speech therapy ${ }^{5}$ & 22 & $86.4 \%$ & & \\
\hline Motor therapy ${ }^{5}$ & 22 & $50.0 \%$ & & \\
\hline \multirow[t]{3}{*}{ GALT gene mutation } & 10 & $50 \%$ & Q188R / Q188R & \\
\hline & 5 & $25 \%$ & Q188R / other ${ }^{6}$ & \\
\hline & 5 & $25 \%$ & other ${ }^{7}$ & \\
\hline
\end{tabular}

GALT enzyme activities indicate that all patients have the classic galactosemia type. Urine galactose and galactitol levels indicate adequate dietary compliance. ${ }^{1}$ GALT activity was measured at diagnosis; ${ }^{2}$ In case the GALT activity is not reported, it was confirmed by the treating physician to be severely decreased; ${ }^{3} \mathrm{ND}=$ not detected; ${ }^{4}$ Urine levels were measured within three months of testing; ${ }^{5}$ At some point in life; ${ }^{6}$ Q188R / L195P $(n=4)$ or Q188R / S135W ( $\left.n=1\right) ;{ }^{7}$ L195P / K229N $(n=3)$ or $400 T d e l / u n k n o w n(n=2)$

\section{Neuropsychological tests}

The Rey Osterreith Complex Figure was used to assess visuo-motor skills (Copy subtest), short term visual memory (Immediate Recall) and long term 
visual memory (Delayed Recall and Recognition) (Meyers and Meyers, 1995). The Bourdon-Vos test was used to measure sustained attention skills (mean RT) (Vos, 1988). The Digit Span (Forward and Backward) addressed verbal working memory skills (van Haasen et al., 1986).

\section{Language paradigm during EEG recording}

Visually animated scenes were presented to the participants. Each scene consisted of three geometrical shapes (square, triangle, or circle) having one of three different colours (red, blue or green). In each trial, one of the three geometrical figures performed an action upon another figure (one figure moves towards or bumps into another figures; described by either 'to fly towards' or 'to bump into'). Participants were asked to either passively watch the scene (control task, ' $C$ ') or to describe the animated scene overtly using one of two possible responses that varied in syntactic complexity: using separate words, 'W' (e.g., "triangle", "red", "square", "green", "to bump into"; minimal syntactic planning) or using sentences, 'S' (e.g., "The red triangle bumps into the green square.", sentence-level syntactic planning) (Indefrey et al., 2001; 2004). Participants were asked to keep the naming format of the phrases constant over trials. In the word 'W' naming format, lexical access of words is required, but virtually no syntactic encoding. In the sentence ' $S$ ' naming format, in contrast, syntactic encoding is required on local noun phrase level (e.g., inflection of adjectives) and on sentence level (e.g., inflection of the verb, determination of the word order, constructing and filling in of the syntactic frame). The control (' $C$ ') condition was added in this study to receive relevant information for the required non-linguistic resources (e.g., visual processes, attention).

\section{Procedure}

The study was conducted in two sessions. In the first session, the neuropsychological tests were carried out in all participants after explanation and written informed consent were given (by the participant and both parents/caregiver). In the second session, the language paradigm and EEG recordings took place. After a brief explanation, participants were prepared and seated in an electrically-shielded, sound-attenuated room in front of a computer monitor. The session started with the control task ' $C$ ', followed by instructions and a practice version of the language task (consisting of 18 practice trials per condition) and the main language experiment.

The main language task consisted of three runs in a blocked design. Each run comprised two blocks which were randomized within the run and counter- 
balanced between participants to exclude order effects. Each block started with a brief instruction reflecting the expected naming format (i.e., either 'SENTENCE' or 'WORD') followed by 32 trials of different scene displays, of which the content (figures, colours, action and arrangement) was randomized. Per condition and participant, a total of 96 trials were recorded. The control task consisted of three consecutive runs, having a total of 108 trials. Figure 3.1 gives a schematic overview of the sequences of events within a trial. The duration of animation in the scene differed (955 or $1885 \mathrm{~ms}$ ) depending on the action format ('to fly towards' or 'to bump into', respectively). The difference in animation durations is caused by a different amount of action frames (10 versus 18 frames, where the actual 'bump' event occurred at frame 14, at $1520 \mathrm{~ms}$ after scene onset). Note that the movements in the scenes are visually identical until they diverge at the moment the 'to fly towards' trials freeze while 'to bump into' trials continue. Participants were instructed to start the description as fast and accurate as possible. The next trials started via a self-paced button push (USBkeyboard key), except for the control trials which had a fixed $2000 \mathrm{~ms}$ interval between trials. Control trials had approximately the same duration as the linguistic trials.

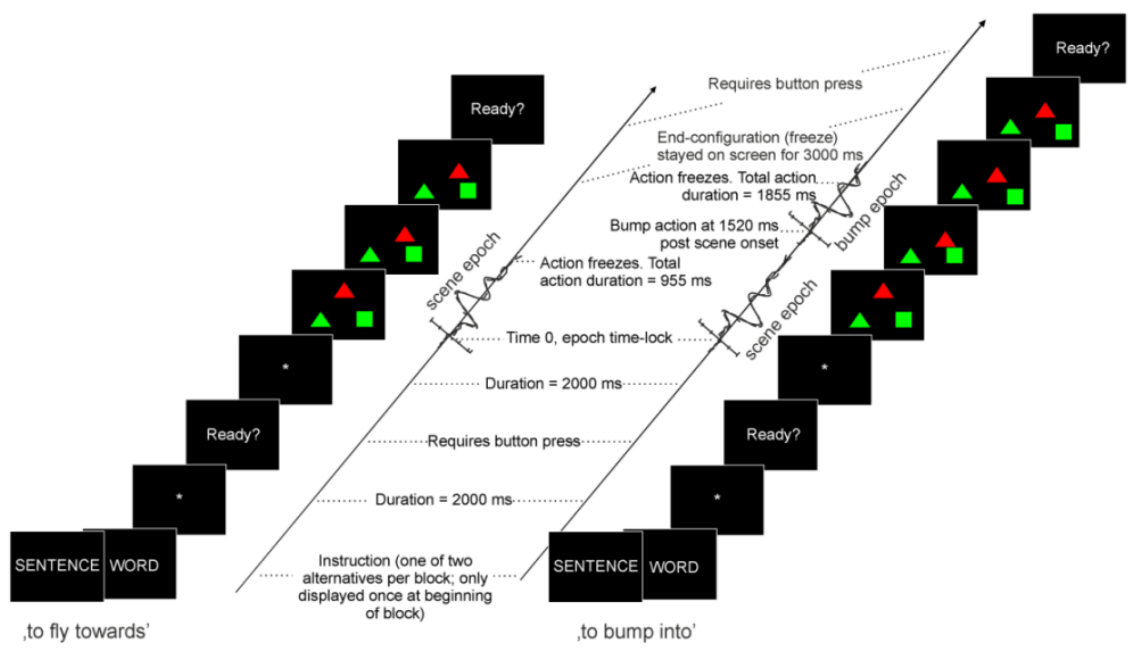

Figure 3.1. Overview of the sequences of events within trials. Timing of events within an experimental trial, separated for the two action formats ('to fly towards' and 'to bump into'). Time is displayed upwards. The block started with the instruction cue ('WORD' or 'SENTENCE'), a fixation cross, a ready sign, and a randomized sequence of trials. For each trail type screenshots are displayed to illustrate the actual moving time period of the objects along with the moments of expected response of the participant, and the corresponding ERP epochs of interest (time-locked to scene onset and the bump event, respectively). 


\section{Electroencephalography (EEG) recording}

The EEG recording was done using an elastic cap in which 32 tin electrodes were mounted (Electro-Cap International $(\mathrm{ECl})$, Inc.), positioned according to the international 10-20 system (Jasper, 1958). Twenty electrodes - F3, Fz, F4, FC3, FCz, FC4, C3, Cz, C4, CP3, CPz, CP4, P3, Pz, P4, O1, Oz, O2, T3 and T4 - were measured as active leads, AFz was used as the ground electrode. The left mastoid (A1) was used as online reference. Offline the signal was rereferenced to the average signal of both mastoids. Vertical eye movements and blinks were monitored by two electrodes placed at the left upper and lower orbital ridge. Horizontal eye movements were recorded with electrodes placed on the left and right cantus. The impedance of all electrodes was kept below 5 $\mathrm{k} \Omega$. Data acquisition was done using Brain Vision Recorder software (Brain Vision, MedCaT B.V.) and the signal was amplified using a $0.05-50 \mathrm{~Hz}$ band pass and sampled at a $500 \mathrm{~Hz}$ interval. The scene onset as well as the voice onset triggered a TTL pulse directly into the EEG recordings. The voice onset pulse was initiated whenever the sound pressure level reached a certain threshold (individually adjusted to each subject) and was transferred via a microphone.

\section{Data analyses}

The number of errors and self-corrections were computed using the recorded audio data and manual (online) scores. Errors were defined as any deviation from the expected utterance (i.e., incorrect figure, colour, action, naming format or ordering). Self-corrections were defined as any overt corrective effort during the response utterance. The voice onset time (VOT) was determined as the time between the scene onset and the onset of the voice response; the total speech time (TST) was cautiously estimated as the time between the onset of the voice response and the button push indicating when participants were ready to continue. VOTs $<0.5$ seconds and $>4.5$ seconds and TSTs $<2$ seconds and $>$ 10 seconds were considered outliers and discarded from analysis. The neuropsychological data were standardized using norm data and classified according to the guidelines of Lezak (1995). A repeated measures General Linear Model was used to analyse the behavioural data (VOT, TST, errors and self-corrections) having Condition ('W' versus 'S') as the within-subject factor and Group (patients, controls) as between-subject factor. The standardized neuropsychological data were analysed using frequency tables (for the classified data) and univariate GLM to examine group differences.

With respect to the EEG data, trials in which the participant's response was absent were excluded from analysis. The EEG data were epoched from -200 to 
$2500 \mathrm{~ms}$ post scene onset (to include the entire interval from onset of visual scene to the end of the display/onset of articulation), band-pass filtered from 0.3-30 Hz (zero phase, $24 \mathrm{~dB}$ ) and baseline corrected (from -200 to $0 \mathrm{~ms}$ ). Large visual artefacts were removed. In addition, data were decomposed using the infomax Independent Component Analysis (ICA) in EEGlab (Delorme and Makeig, 2004). This method disentangles brain- and artefact-related processes by searching for maximally independent components (Makeig and Onton, 2009). Stereotype artefact-related components reflecting eye movements, noise and muscle activity were subsequently removed. On average, $84.5 \%$ of all trials (SD 5.2\%) were kept for analysis [no difference between groups, $F_{1,41}=.00, p=$ .988]: mean 96 trials in ' $C$ ', 79 in ' $W$ ' and 78 in ' $S$ '. The remaining components (the cleaned data) were back-projected into the ERP. In the back-projected ERPs, epochs were divided in two time ranges: one interval related to the scene onset (-200 to $1000 \mathrm{~ms}$ after scene onset), and one related to the bump event (200 to $800 \mathrm{~ms}$ after the bump event, or 1320 to $2320 \mathrm{~ms}$ post scene onset) (see also Figure 3.1). Note that in the bump epoch, only 'to bump into' trials were included (and no 'to fly towards' trials), corresponding to on average 49 trials in ' $C$ ', 39 in ' $W$ ' and 40 in ' $S$ '. The bump epochs were baseline corrected (-200 to 0 ms after the bump event). Based on visual inspection of the grand averages, target peak ERP components and corresponding time windows were specified on which we conducted mean amplitude analyses.

ERP statistics were performed on the mean amplitude data per time window, condition, and participant using repeated measures GLM with Condition as within-subjects factor ('C', 'W', 'S'), and two within-subject topographical factors Laterality (left, central, right) and Anterior-Posterior (F, FC, C, CP, P, O). Based on visual inspection, additional analyses were performed on subsets of electrodes. Group was added as the between-subjects factor (patients, controls). Pearson's correlations were used to examine the relationship between the ERP data and behaviour (online measures of reaction times and accuracy) and other cognitive functions (offline neuropsychological tests); and with patient characteristics (e.g., mutation, rest activity of the enzyme). Where necessary, corrections were made for multiple testing (Bonferroni) and for sphericity violations (Greenhouse Geisser). Age and gender were added as covariates in all analyses but the ones performed on standardized data. An alpha of 0.05 was used as significance level. 


\section{Results}

\section{Neuropsychological test results}

The patients scored significantly lower compared to controls on the following subtests: Rey Complex Figure Copy, Immediate and Delayed Recall and Recognition; Bourdon-Vos total RT and number of errors; and Digit span [.000 < $p<.027]$. However, when the Rey Complex Figure Copy score was subtracted from the Immediate Recall score (not standardized, to control for visuo-motor differences), the groups did not differ [ $p=.75]$. Examining the slope of the three Bourdon-Vos RTs (not standardized, to examine the sustainability of attention), the groups did not differ either $[p=.25]$. The groups did not differ on the number of omissions and corrections on the Bourdon-Vos $[p=.91$ and $p=.33$, respectively]. Table 3.2 gives an overview of the neuropsychological data of the patient group (control data is not presented for clarity reasons).

Table 3.2. Classified neuropsychological data of the patients with galactosemia

\begin{tabular}{|c|c|c|c|c|c|c|c|}
\hline & $\begin{array}{l}\text { Very } \\
\text { low }\end{array}$ & Low & $\begin{array}{c}\text { Below } \\
\text { average }\end{array}$ & Average & $\begin{array}{c}\text { Above } \\
\text { average }\end{array}$ & High & $\begin{array}{l}\text { Very } \\
\text { high }\end{array}$ \\
\hline $\begin{array}{l}\text { Expected } \\
\text { distribution }\end{array}$ & $2.3 \%$ & $7.4 \%$ & $17.7 \%$ & $45.2 \%$ & $17.7 \%$ & $7.4 \%$ & $2.3 \%$ \\
\hline \multicolumn{8}{|l|}{$\begin{array}{l}\text { Rey Complex } \\
\text { Figure }\end{array}$} \\
\hline Copy & $68.2 \%$ & $13.6 \%$ & $18.2 \%^{1}$ & & & & \\
\hline $\begin{array}{l}\text { Time to } \\
\text { copy }\end{array}$ & - & $8.3 \%$ & $91.7 \%^{1}$ & & & & \\
\hline $\begin{array}{l}\text { Immediate } \\
\text { Recall }\end{array}$ & $59.1 \%$ & $22.7 \%$ & $9.1 \%$ & $4.5 \%$ & $4.5 \%$ & - & - \\
\hline $\begin{array}{l}\text { Delayed } \\
\text { Recall }\end{array}$ & $54.5 \%$ & $27.3 \%$ & $9.1 \%$ & $9.1 \%$ & - & - & - \\
\hline Recognition & $27.3 \%$ & $18.2 \%$ & $18.2 \%$ & $31.8 \%$ & $4.5 \%$ & - & - \\
\hline \multicolumn{8}{|l|}{ Bourdon-Vos } \\
\hline Total RT & - & $59.1 \%$ & $27.3 \%$ & $13.6 \%$ & - & - & - \\
\hline
\end{tabular}



Number of
omissions
$59.1 \%$
$36.4 \%$
$4.5 \%$
Number of
corrections
$4.5 \%$
$45.5 \%$
$50.0 \%$
Number of
errors
$36.4 \%$
$63.3 \%$
Digit Span

$$
42.9 \% \quad 23.8 \% \quad 14.3 \% \quad 19.0 \%
$$

Presented are the percentages of patients scoring within the particular classifications as described in Lezak (1995): $z<-2$ very low; $-2<z<-1.3$ low; $-1.3<z<-0.6$ below average; $-0.6<z<0.6$ average; $0.6<z<1.3$ above average; $1.3<z<2$ high; $z>2$ very high. Note that the expected distribution reflect percentages based on the normal distribution. ${ }^{1}$ Below average or higher

\section{Behavioural data language paradigm}

\section{Accuracy}

The number of errors differed between groups, $\left[F_{1,39}=12.24, p=.001\right]$ : the patients made more errors than the controls. There was no difference in the number of errors between the word ' $W$ ' versus the sentence ' $S$ ' condition $\left[F_{1,39}\right.$ $=2.14, p=.151]$. The number of self-corrections showed no group difference $\left[F_{1,39}=0.06, p=.801\right]$, but a condition effect. More self-corrections were made in 'S' compared to 'W' $\left[F_{1,39}=27.78, p<.001\right]$ (Figure 3.2).

\section{Reaction times}

The patients had longer VOTs and longer TSTs compared to controls $\left[F_{1,37}=\right.$ 5.28, $p=.027$ and $F_{1,37}=13.15, p=.001$, respectively]. The TST was longer in ' $S$ ' $\left[F_{1,37}=26.41, p<.001\right]$. No condition effect for the VOT was observed in either group $\left[F_{1,37}=.06, p=.807\right]$.

\section{Correlations behavioural data and neuropsychological data}

In both groups, lower scores on the Rey Complex Figure (Immediate and Delayed Recall) were related to more errors [patients: $-.543<r<-.490, .009<p$ $<.021$; controls: $-.550<r<-.478, .010<p<.028$ ]. In patients, lower performance on the Rey Immediate recall task was associated with longer TSTs $[r=-.651, p=.001]$. 
Errors

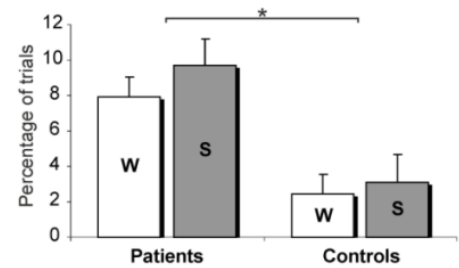

Self-corrections

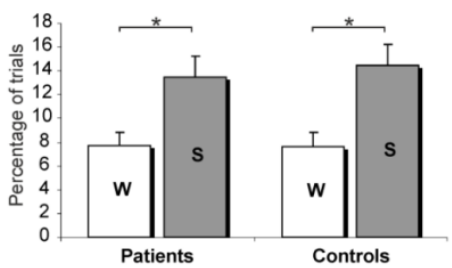

Voice onset times

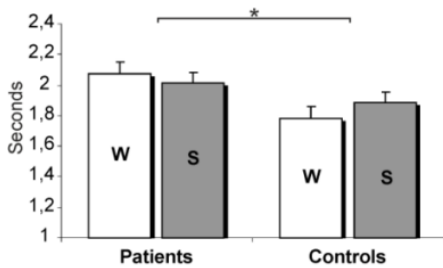

Total speech times

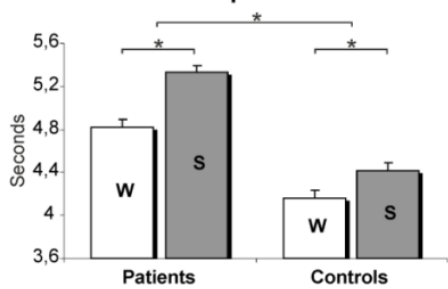

Figure 3.2. Behavioural data. Behavioural data per group and per condition. 'W' = Word condition; ' $S$ ' = Sentence condition. Presented are estimated marginal means with standard error (SE) bars. Asterisks indicate significant effects $(p<.05)$.

\section{ERP data}

The ERP waveforms depict the planning phase of the utterance from scene onset onwards. Figure 3.3 shows the grand average waveforms of the patients with galactosemia versus the matched controls for the entire epoch interval of 200 to $2500 \mathrm{~ms}$ after scene onset (averaged across conditions). Separate lines are shown for the two action formats 'to fly towards' and 'to bump into'. The figure illustrates that the scenes (and the corresponding ERPs) were identical until approximately $1000 \mathrm{~ms}$ post scene onset and start to diverge relatively late. Visual inspection of the grand averages showed a clear ERP morphology during the first thousand milliseconds post scene onset, followed by a relatively steady period (in which no event-related activity is visible). Another subset of ERP components was observable at a relatively late time interval (from approximately $1500 \mathrm{~ms}$ after scene onset onwards), restricted to the bump trials. Analyses were directed towards these two epochs of interest: -200 to $1000 \mathrm{~ms}$ after the scene onset (before the action format and thus the verb is available; local syntactic planning) and -200 to $800 \mathrm{~ms}$ after the bump event (when the verb is available, corresponding to 1320 to $2320 \mathrm{~ms}$ after scene onset, limited to the bump trials; global sentence-level syntactic planning). As the arrows in Figure 3.3 depict, there are several time points at several electrodes where groups and/or conditions differ, starting early in time. The 
overall morphology, however, was quite similar (see also topographies in Figure 3.3). Statistical analyses were carried out across several time windows with labels 'scene' referring to components following scene onset, and label 'bump' referring to components following the 'bump' event: $90-150 \mathrm{~ms}$ (referred to as $P 1$ scene), 100-160 ms (N1 scene), 180-240 ms ( $P 2$ scene) and 350-650 ms ( $P 3$ scene) post scene onset; 70-170 ms (N1 bump), 180-280 ms ( $P 2$ bump) and $300-500 \mathrm{~ms}$ ( $P 3$ bump) post bump event. Note that the labels P1, N1, P2 and $\mathrm{P} 3$ are used for descriptive purposes.

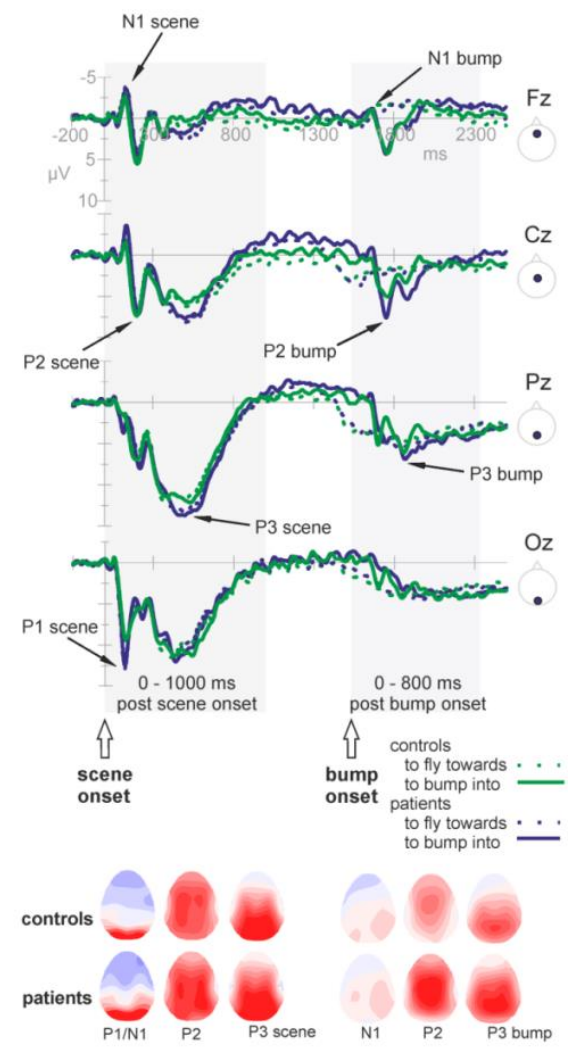

Figure 3.3. Overview of ERPs and topographies. Top: Grand average ERPs of the patients with galactosemia (blue) and the healthy controls (green) across the midline of the scalp ( $F=$ Frontal, $C$ $=$ Central, $\mathrm{P}=$ Parietal, $\mathrm{O}=$ Occipital). The lines are averaged across conditions, but separate for the two action formats: solid lines represent the 'to bump into' format; dashed lines the 'to fly towards' format. The two epochs of interest are highlighted: the post scene onset epoch (where scenes of both action formats, and their corresponding ERPs, are still identical) and the post bump event epoch (where the analyses was limited to the 'to bump into' trials, as the 'to fly towards' trials do not show an ERP morphology during this time window). Negative voltage is plotted up in this and all subsequent figures. Bottom: Overview of the topographical distributions over the scalp of the 
components of interest, separate for each group. Both the ERPs and the corresponding topographies illustrate an overall similar morphology for the patients and controls.

\section{Time windows of interest post scene onset}

\section{Time window 90-150 ms - P1 scene}

A positive component was observed with a maximum around $120 \mathrm{~ms}$ post scene onset and an occipital scalp distribution. Analyses were restricted to the occipital plane (O). A Group effect $\left[F_{1,39}=6.00, p=.019\right]$ was visible and significant in all three conditions ('C': $p=.032$, 'W': $p=.021$, ' $\mathrm{S}$ ': $p=.021$ ), with the patients' ERP being more positive. The patients but not the controls showed a trend in the Condition effect $\left[F_{1.6,30.2}=2.91, p=.081\right]$. Simple contrasts in the patients data revealed that ' $C$ ' differed from both ' $W$ ' and ' $S$ ' $[p=.004$ en $p=$ .024 , respectively] (see Figure 3.4A).

\section{Time window 100-160 ms - N1 scene}

At anterior sites, a negative component was observed at 100-160 ms after scene onset, with a maximum at $130 \mathrm{~ms}$. Analyses were restricted to F3, Fz, F4. No clear condition effect was revealed. The Group effect was not significant either $\left[F_{1,39}=3.47, p=.070\right]$.

\section{Time window 180-240 ms - P2 scene}

A positive component was observed peaking around $210 \mathrm{~ms}$ after scene onset. Analyses showed that this component was largest over midline fronto-central and parietal sites. Because of interactions between Condition and the topographical factors, the analysis was further conducted on sub-regions.

At the right side of the scalp (F4, FC4, C4, CP4, P4) there was a Condition effect $\left[F_{2.0,76.0}=7.93, p=.001\right]$. Follow-up analyses showed that ' $C$ ' differed from both ' $W$ ' and ' $S$ ' $[p=.001$ and $p=.007$, respectively]. There was no difference between ' $W$ ' and ' $S$ '. Recordings at right posterior electrodes (CP4, P4) revealed a Group effect [ $\left.F_{1,39}=4.62, p=.038\right]$. Follow-up analyses showed that the patients' ERP signal in the linguistic conditions (but not in passive watching) was more positive compared to controls ['W': $F_{1,39}=4.31, p=.044$; 'S': $F_{1,39}=4.97, p=.032$ ] (see Figure 3.4B).

Only in controls, better sustainability of attention (lower slope of the Bourdon Vos reaction times) was associated with a larger linguistic condition effect (i.e., difference in mean amplitudes between ' $C$ ' and both ' $W$ ' and ' $S$ ' at FC4) ['C'-'W': $r=-.444, p=.044$; 'C'-'S': $r=-.754, p<.001]$. 


\section{Time window 350-650 ms - P3 scene}

During this time window, a large long-lasting positive activity was observed, with a maximum at posterior sites. Analyses indicated interactions between the Condition effect and the electrode locations. Therefore, further analyses were performed on sub-regions.

In central and parietal regions (FC, C, CP, P), a Condition effect was observed [ $\left.F_{2.0,77.3}=21.19, p<.001\right]$. Pair wise comparisons showed that ' $\mathrm{C}$ ' differed from 'W' and ' $\mathrm{S}$ ' [both $p<.001$ ]. The groups did not differ significantly and the Condition effect was the same for both groups. At frontal regions $(F)$, a difference was observed between ' $S$ ' and ' $W$ ' $\left[F_{1,39}=4.84, p=.034\right]$. However, this effect was only present in the controls $\left[F_{1,18}=7.59, p=.013\right]$ and not in the patients $\left[F_{1,19}=.02, p=.884\right]$ (see Figure 3.4C). At the frontal site, there is a trend towards a difference in amplitudes between groups $\left[F_{1,39}=2.63, p=.113\right]$.

In controls, larger syntactic complexity effects (i.e., mean amplitude difference between ' $S$ ' and 'W' at Fz) were associated with shorter TSTs (in 'S') [ $r=-.462$, $p=.035]$. In patients, longer VOTs (in 'S') were associated with smaller syntactic complexity effects $[r=-.474, p=.030]$.

\section{Time windows of interest post bump event}

\section{Time window 70-170 ms - N1 bump}

At anterior sites, a negative component was visible at $70-170 \mathrm{~ms}$ after the bump event, on average peaking at $130 \mathrm{~ms}$. Analyses were restricted to frontal and fronto-central electrodes ( $F$ FC). There was no significant Condition effect $\left[F_{1.8}\right.$, $71.3=1.96, p=.151]$, nor any interaction effects. The groups did not differ either $\left[F_{1,39}=0.65, p=.43\right]$.

\section{Time window 180-280 ms - P2 bump}

Around 230 ms post bump event, a large positive component was observed. The topographic distribution was fronto-central. Analyses therefore were restricted to these electrodes (F FC C). In addition to Condition and Group effects, interaction effects were found between the factor Anterior-Posterior and both Group and Condition.

Sub-analyses revealed only a marginal Condition effect in the $\mathrm{FC}$ plane $\left[F_{2.0,77.9}\right.$ $=2.94, p=.059]$, while the Group effect was significant $\left(F_{1,39}=9.42, p=.004\right]$. In the central plane $(\mathrm{C})$, there was a clear Condition effect $\left[F_{2.0,77.7}=9.24, p<\right.$ .001], where ' $\mathrm{C}$ ' differed from both ' $\mathrm{W}$ ' and ' $\mathrm{S}$ ' $[p=.000$ and $p=.006$, respectively]. The two linguistic conditions did not differ $[p=.214]$. The Group 
effect was significant $\left[F_{1,39}=12.88, p=.001\right]$, reflected in more positive amplitudes in the patients' ERP compared to controls (see Figure 3.5A).
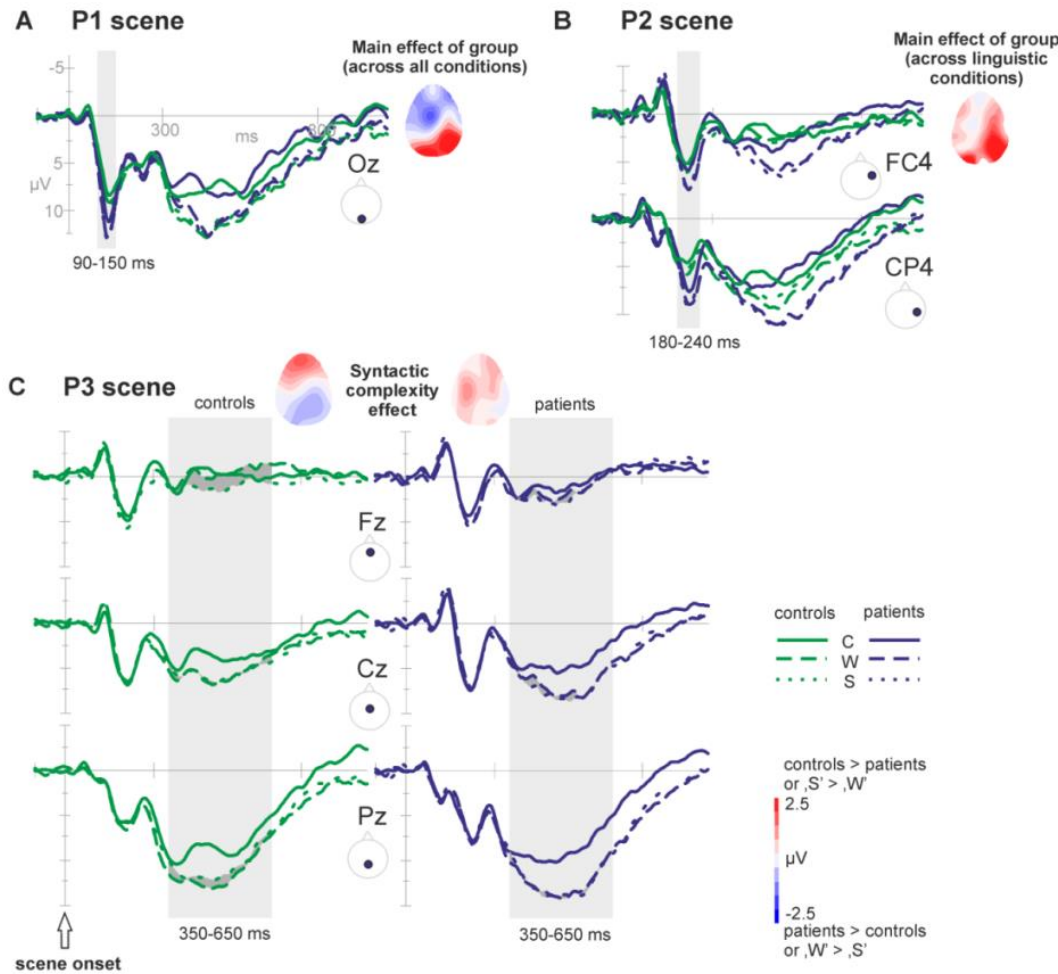

Figure 3.4. Overview of the ERP effects in the post scene onset epoch. A) Grand average waveforms of the occipital midline electrode $(\mathrm{Oz})$ displaying the $\mathrm{P} 1$ Group effect (patients $>$ controls) within the time window 90-150 ms post scene onset. This group effect (difference between groups) is also displayed in the topography. B) Grand average waveforms of two right-hemispheric electrodes (i.e., FC4, CP4) displaying the P2 Group effect (patients > controls; also displayed in the topography) and the lexical access effect ('W'='S' > 'C') within the time window 180-240 ms post stimulus. C) Grand averages of the anterior P3 syntactic complexity effect (' $S$ ' > 'W'; highlighted in dark grey), significant in controls but not in patients, within the time window $350-650 \mathrm{~ms}$ post scene onset. The corresponding topographies of the syntactic complexity effect also show the effect in the controls, while no clear effect is observable in the patients.

Only in controls, larger linguistic condition effects (i.e., difference between nonlinguistic and linguistic conditions at $\mathrm{Cz}$ ) were associated with shorter VOTs [' $\mathrm{C}$ ''W' effect: $r=-.497, p=.033$; 'C'-'S' effect: $r=-.633, p=.002$ ] and with fewer errors ['C'-'W' effect: $r=-.517, p=.016$; 'C'-'S' effect: $r=-.602, p=.004$ ]. Only in patients, better visual memory performance (Rey Complex Figure Immediate 
and Delayed Recall) was related to larger linguistic condition effects ('C'-'W') [ $r$ $=.570, p=.006 ; r=.611, p=.003$, respectively].

\section{Time window 300-500 ms - P3 bump}

Between 300 and 500 ms post bump event, a large positive component was observed. The component was broadly distributed, with a maximum over posterior sites (CP P). Analyses were performed on F FC C CP P electrode lines. In addition to a significant Condition effect, there were interactions between the Anterior-Posterior topographical factor and the Condition factor. The groups differed across the entire scalp $\left[F_{1,39}=11.21, p=.002\right.$; in all conditions, 'C': $p=.046$, 'W': $p=.010$, 'S' $p=.001]$.

Sub-analyses revealed a Condition effect that was present in all planes (except for $\mathrm{F}$ ), but was largest at posterior sites [P: Condition $\left.F_{2.0,76.0}=9.08, p<.001\right]$. Follow up analyses showed that ' $\mathrm{C}$ ' differed from both ' $\mathrm{W}$ ' and ' $\mathrm{S}$ ' $[p<.001$ and $p=.002$, respectively]. Posterior, the linguistic conditions did not deviate. Anterior, however, ' $\mathrm{W}$ ' and ' $\mathrm{S}$ ' differed significantly [FC: $p=.025$ ], with ' $\mathrm{S}$ ' being more positive than ' $W$ '. There was no interaction between Group and Condition (see Figure 3.5B).

Only in controls, better sustainability of attention was associated with larger syntactic complexity effects (i.e., difference in mean amplitude between ' $S$ ' and 'W' at FCz) [ $r=.498, p=.022]$.

\section{Associations between outcome data and patient characteristics}

There were no significant correlations between the patient characteristics (i.e., age at introduction diet, GALT enzyme activity, urine galactose and galactitol values) and the ERP data. Inspection of correlations with behavioural data revealed that older age at introduction of diet was related to longer TSTs [ $r=$ $.689, p=.001]$. Further, higher urine galactitol values were correlated with the shorter VOTs $[r=-.514, p=.017]$. Better verbal working memory scores (Digit Span) were related to lower galactitol values in urine [ $r=-.471, p=0.031]$. Differential effects for patients with different genotypes were explored (homozygous for Q188R versus other mutations). The GALT enzyme activity and urine galactose and galactitol values did not differ across groups. The Q188R homozygous group had longer VOTs [only in the ' $W$ ' condition, $F_{1,18}=$ $5.21, p=.036]$. No differences in neuropsychological scores were found, but the groups differed with respect to the ERP effects: the syntactic complexity effect (i.e., difference between the linguistic conditions in the P3 bump time window) was greater in the 'other mutation' group compared to the homozygous group $\left[F_{1,19}=13.36, p=.002\right]$. 
A P2 bump

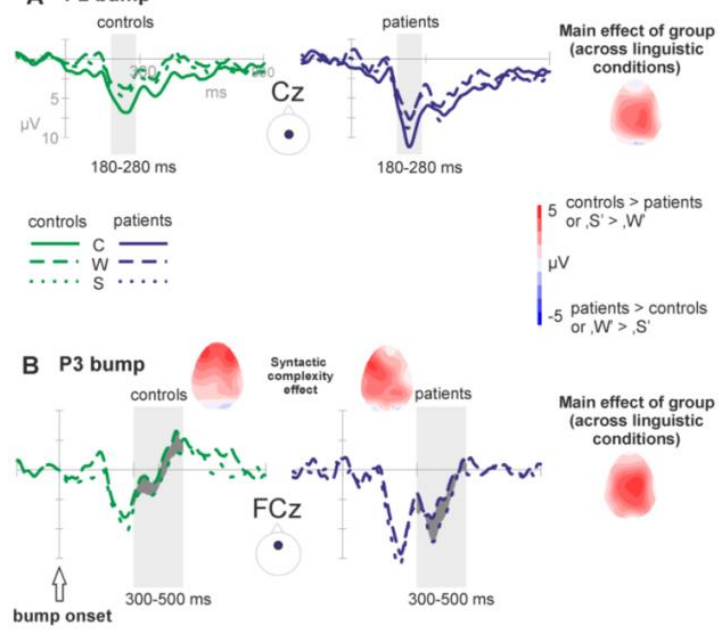

Figure 3.5. Overview of the ERP effects in the post bump event epoch. A) Grand average waveforms of the central midline electrode $(\mathrm{Cz})$ displaying the P2 bump group effect (patients > controls; also reflected in the topography) and the P2 bump linguistic condition or lexical access effect ('W'='S' < 'C') within the time window 180-280 ms post bump event. B) Grand averages of the fronto-central midline electrode ( $F C z)$ reflecting the P3 bump syntactic complexity effect ('S' > 'W'; reflected in the dark grey fill in the ERPs and in the corresponding topographies) and the P3 group effect (patients > controls; see also topography) within time window 300 - 500 ms post bump event.

\section{Discussion}

This study is the first to apply theories, methods, and experimental paradigms from cognitive neuroscience to study language production impairments in classic galactosemia. This approach reveals impairments in several language production stages prior to articulation in these patients.

\section{Behavioural data}

The adolescent participants described animated scenes using different syntactic complexity formats: either separate words ('W') or complete sentences ('S'). Both groups required more self-corrections and speaking time in the sentence condition as compared to the word condition, suggesting that the intended complexity variation of syntactic planning was successful. Several outcome measures are found to deviate in patients compared to matched controls. The patients made more errors than controls ( $8.8 \%$ versus $2.8 \%$ of all trials). They needed more time to prepare (VOT 2.0 versus 1.8 seconds) and to finish the utterance (TST averaged across conditions: 5.1 versus 4.3 seconds), indicating 
that the patients were both slower and less accurate. Interestingly, in both groups, the error rates and voice onset times did not differ across the sentence and the word condition. The finding that the speaking time is modulated by syntactic complexity, but the voice onset time is not, suggests that most of the syntactic planning occurs after the initiation of the utterance.

\section{ERP components of healthy controls}

The ERPs reflect the entire information processing sequence, including visual processing of the figures and their movements, and the language planning process. We will first discuss the effects of the condition modulations in the control group only, in order to make inferences on their functional relevance. Several time windows showed a condition modulation, before and after the action format (the verb) became clear, reflecting the early initiation of the utterance and the incremental nature of the language planning.

The P1 scene is, with respect to distribution and latency, most likely an instance of the occipital $\mathrm{P} 100$, traditionally associated with visual and attention processes (Coles and Rugg, 1995; Luck, 2005). The P1 has also been linked to motion processing of visual stimuli (i.e., influenced by on- and offset, linked to V1, Mercier et al., 2009) and to conceptual processes (Rabovsky et al., 2012; Rahman and Sommer, 2008). There was no modulation with condition, indicating similar requirements for motion processing, attention and conceptualisation across the conditions in this study. The P2 scene component is most likely a P200, traditionally observed over anterior sites (Luck, 2005) and linked to lexical access of words during picture naming (e.g., Costa et al., 2009; Strijkers et al., 2009) or word reading (Bles et al., 2007). Along this line, the observed effect likely reflects lexical access, as the linguistic conditions ('W' and ' $S$ ') do not differ in lexical requirements or P2 modulation, but differ from passive viewing (not requiring lexical access). Although the scene just started at this point, it is already clear which figure is the actor and which object is involved in the action, while the action format - verb - is still ambiguous. Therefore, lexical access is most likely restricted to access of the first noun phrase (actor). The idea that planning starts with the onset of the visual stimuli is consistent with the idea that language production is (at least partly) driven by visual input or visual attention (Bock, 1982; Meyer and Lethaus, 2004; see also Mortensen et al., 2008). The long lasting and widely distributed P3 scene resembles a P300. Anterior, the P3 showed variation with syntactic complexity. The timing and direction of the effect is in line with previous reports of the P300 reflecting integration of working memory and attention, both necessary for updating incoming information over time (e.g., Kok, 2001). The observed ERP variation 
with syntactic complexity at the frontal midline (where ' $S$ ' is more positive than 'W') can be explained by the need for more attention- and memory-related resources in case of higher syntactic complexity, or could be a direct indication for more complex syntactic processing. At this time point, the action format (verb) is still ambiguous as differentiation between the two potential verbs can only happen after appropriate visual input (the bump, at $1520 \mathrm{~ms}$ after scene onset). Based on this, we conclude that syntactic planning reflected by the P3 must be restricted to local syntactic processing (i.e., retrieval of syntactic information about the actor/noun, inflection of the adjective). As there was no syntactic complexity effect in the VOT, we can assume that the utterance is initiated prior to syntactic planning once the first element of lexical access is in (noun or noun phrase) (Allum and Wheeldon, 2007; Allum and Wheeldon, 2009). Larger P3 syntactic complexity effects were associated with shorter TSTs, indicating that more advanced local syntactic planning decreases the speaking time or increases the efficiency of the language process. In the following time window, the ERP shows activity around baseline (approximately 900 to 1400 ms post scene onset), presumably reflecting neural activity without clearly measurable events (eventually due to high variation in cognitive processing within and between groups). Then, divergence across action formats (verbs) occurs both scene-wise and ERP-wise. Time-locked to the moment of the bump, another set of ERP components arise (in the 'to bump into' trials only, presumably because the lack of a clear temporal event in the 'to fly towards' trials). During the fronto-central P2 bump component, we observed a condition pattern identical to that of the $P 2$ scene component: the two linguistic conditions differed from passive watching, but not from each other. Now, all information is available (including the verb), making lexical access of the verb possible in an unambiguous way. Larger linguistic condition effects (i.e., difference between non-linguistic and linguistic conditions) were related to shorter VOTs and less errors, indicating that larger linguistic condition effects are associated with more accurate and faster performance. Finally, the large and widely distributed P3 bump component probably reflects a P300. Again, this post-bump P3 showed a similar pattern as the post-scene P3: variation with syntactic complexity. At this point, not only local but also global syntactic planning is required in the sentence condition (i.e., combination and integration of all noun phrases and the verb into a well-formed sentence), reflected in the larger P3 amplitudes. To sum, the functional interpretation of the ERPs in healthy controls is such that it starts with a set of components related to processing of moving visual information/conceptualisation ( $P 1$ scene), lexical access of the noun phrases $(P 2$ scene) and local syntactic planning of the noun phrases ( $P 3$ scene). When all information, including the verb, is available, the ERP continues with similar 
components related to lexical access of the verb ( $P 2$ bump) and to syntactic planning on a more global sentence-level ( $P 3$ bump).

Relatively few studies have examined overt naming during ERPs recording, especially not using multi-word utterances (e.g., Jansma et al., 2004; Koester and Schiller, 2008; Marek et al., 2007; Strijkers et al., 2009). Marek et al. (2007) elicited multi-word utterances and sentences and found a posterior P3-like component (350-500 ms post stimulus) reflecting syntactic complexity (in addition to increasing conceptual complexity, as the used paradigm did not disentangle the two). In the present study, conceptual complexity was kept constant, suggesting that the observed P3 modulations speak to syntactic complexity proper. The production P3's that are found in the current study and the described previous studies, might therefore be analogous to the P600/SPS in syntactic comprehension (Hagoort et al., 1993; Kaan et al., 2000; Osterhout and Holcomb, 1992), albeit with a more anterior distribution of the syntactic effect. The finding that both instances of the P3 in this study (post scene and post bump event) display the syntactic complexity effect provides additional support for a role for syntactic encoding in this component.

Psycholinguistic models of speech processing assume incremental planning of an utterance (e.g., Bock, 1995; Levelt, 1989; Meyer, 1996). In our study, the utterance seems to be initiated after lexical access of the first noun, but prior to syntactic planning of this noun phrase (as the VOT did not vary with syntactic complexity). Our results therefore support the idea that an utterance can be initiated before the visual stimulation is finished (and before all necessary information is available). In addition, by means of ERP variations, we could look into the linguistic planning phase. Well before voice onset and before the visual input of the scene is complete, we observed activation related to local syntactic planning. When all information was available, there was continuation of syntactic encoding (on a more global, sentence-level). Although this paradigm was originally implemented using PET (Indefrey et al., 2001; 2004), this study demonstrates its suitability for high temporal resolution methods, since it allows us to disentangle this early local and later more global planning.

\section{ERP components of patients with classic galactosemia}

The patient ERPs showed a similar morphology compared to that of the matched controls, suggesting a generally intact neural network of cognition and language processing. The patient ERP differed from those of controls in several time windows. In the P1 scene component, related to attention, visual integration of moving objects and conceptualisation processes, the patients showed higher amplitudes in all three conditions (classically interpreted as more 
effortful processing) compared to controls. The fact that the patients differed in all conditions from controls, including passive watching, suggests early visual or attention processing deficits or an increased effort to integrate moving objects over time. Moreover, the patients showed a difference between linguistic and non-linguistic conditions ('W'/'S' versus ' $C$ ') that was not present in controls, suggesting linguistic effects in this early time window, likely reflecting impaired conceptualization. This is the first evidence that the patients diverge at an early stage in cognitive information processing from healthy controls during the preparation of language. In the P2 scene, associated with lexical access, the patients showed the same pattern of condition effects as the controls (difference between control condition and both language conditions). Posterior, the patients showed greater amplitudes in both language-related conditions compared to controls, suggesting difficulties with lexical access. During the P3 scene, the patients did not show the syntactic effect. The finding that the controls showed this syntactic variation but the patients did not can be interpreted as a ceiling effect for the patients: the sentence condition does not diverge from the word condition, as the ceiling level of memory/attention resources is already reached in the word condition (descriptively corroborated by the grand averages showing that in the patient ERP both the ' $W$ ' and ' $S$ ' condition are in the same range as the ' $S$ ' condition in controls). It could be that the patients perform less efficient advance syntactic planning. In controls, larger syntactic complexity effects (i.e., more advance syntactic planning) were associated with shorter TSTs. The patients needed more speaking time compared to controls, also indicative of less (efficient) advance syntactic planning. Different from the controls, smaller syntactic complexity effects were related to longer VOTs, suggesting that for the patients, less advance syntactic planning is related to slower or later initiation of the utterance. The patient' ERP further deviates from controls in the P2 bump component, providing additional support for impaired lexical access in the patients. Finally, the groups differed from each other during the P3 bump component, with the patients having larger mean amplitudes compared to controls. The syntactic variation was also present in the patients (opposite to the P3 scene component, where only the controls showed this variation). Two explanations are: they require more resources when engaging sentence-level syntactic planning (explaining the higher amplitudes) or, they compensate for earlier impairments in local syntactic planning by engaging in both local and sentence-level syntactic planning at a later (post bump) planning phase causing the higher amplitudes. We cannot disentangle between these alternatives at the moment.

The finding that there were no significant (or minor) differences in the ERP morphology between the groups in passive watching confirms an overall 
comparable cognitive system, suggesting that behavioural language impairments of the patients are not part of a severe general impairment. This assumption receives empirical support by the observed difference in ERPs between groups for the linguistic task. These differences cannot be explained merely by differences in the visual processing between conditions, but must be related to higher language function - as this was the task manipulation. Besides language planning the effects could be explained by variation in attentional or memory resources. Such variation across different naming formats cannot be excluded.

We investigated whether the observed impairments were purely linguistic in nature or whether they can be explained by other cognitive difficulties by looking into their neuropsychological test profile and by comparing the ERP with test results of specific cognitive functions. As reported in the result section and consistent with previous reports (Antshel et al., 2004; Doyle et al., 2010; Schweitzer et al., 1993; Waggoner et al., 1990), the patients scored lower on several neuropsychological tests. The patients were slower (Bourdon-Vos reaction times, Vos, 1988) and had difficulties with the visuo-motor task (Rey Complex Figure Copy subtest, Meyers and Meyers, 1995, among other things requiring the integration of a multitude of components into a unifying whole). Important too is that visual working memory, when corrected for the visuo-motor differences, was not significantly worse in the patients. Therefore, visual working memory (keeping the visual scene online and actively in mind) cannot explain the behavioural and ERP-related differences between the groups. Verbal working memory performance (van Haasen et al., 1986), however, was lower in the patients, potentially adding to the language impairments. Importantly, verbal working memory scores were not correlated to the behavioural and ERP effects during the language task. The lack of correlation suggested that verbal working memory did not directly contribute to the observed ERP effects. Interestingly, several domains that are affected in the patients with classic galactosemia (i.e., visuo-motor skills, motion processing) require some form of integration of information over time. Such an integration deficit may also lead to the difficulties in constructing syntactic frames as well as difficulties to access and fill in the right words into these frames (Bock and Levelt, 1994).

Correlations with patient variables (i.e., GALT enzyme activity, age at introduction diet, urine galactose and galactitol values) were far from robust, consistent with previous studies failing to find predictive value for these variables (e.g., Kaufman et al., 1995; Shield et al., 2000; Waggoner et al., 1990). We observed that patients homozygous for the Q188R mutation 
performed worse on certain aspects compared to patients with other mutations, which is in line with other, but not all, studies (Shield et al., 2000). Patients with the Q188R/Q188R mutation had longer VOTs and showed smaller syntactic complexity effects in the P3 bump ERP component.

Previous studies in classic galactosemia have reported general cognitive slowing and diffuse white matter abnormalities (Dubroff et al., 2008; Nelson et al., 1992; Widhalm et al., 2002), theoretically linked to deficient galactosylation of cerebrosides (an important component of myelin) (Lebea and Pretorius, 2005). In line with these findings, our study showed longer reaction times for the patients (both the time needed to prepare and to finish the utterance). In the ERP data, we did not find any delays in the overall evolution of the ERP components. The morphology of the signal was similar for patients and controls. We observed amplitude differences, suggesting an alteration in the neural activity related to a certain cognitive processing phase, which indicates that brain abnormalities might be more clustered than previously suggested. Within the P3 scene time window we see a comparable onset of the component, but the $\mathrm{P} 3$ seems to be extended in time for patients compared to matched controls. As depicted in Figure 3.4C, for controls the ERP signal for the ' $W$ ' and ' $S$ ' conditions catches up with the signal of the ' $C$ ' condition sooner, especially more posterior. This overall ERP pattern of the patients suggests that the local neural circuits work within time windows that are comparable to those of healthy controls. However, the larger amplitudes in the patient ERPs indicate aberrant neural activation patterns. Accumulating metabolites or resulting deficiencies that alter neuronal signalling might be involved herein (e.g., myo-inositol, Berry, 2011). In contrast, the overall integration problem might result from problems of long distance neural communication possibly associated with myelin abnormalities compromising information transfer (Dubroff et al., 2008; Nelson et al., 1992). However, whether abnormal cell signalling and/or brain connectivity is affected and in which specific regions, requires further investigation.

To summarize, patients with classic galactosemia show difficulties in this language production task, both behaviourally (less accurate and slower) and in their ERPs, compared to healthy controls. The ERP deviations start already around the time that attention is directed towards the relevant, moving objects and conceptual knowledge of these objects becomes available, suggesting that these processes are affected by the disease. The ERP differences continue through the consecutive linguistic preparation phases, indicating affected lexical access and impaired syntactic planning (both local and sentence-level syntactic planning). We conclude that, although anecdotal reports have appeared on weak word retrieval and sentence construction, this study is the first to provide 
neurocognitive evidence for language impairments in patients with classic galactosemia. These impairments affect the planning of language, which occurs prior to the output stage. Based on the ERP data, we suggest that these impairments are related to problems in lexical access and syntactic planning of an utterance. These findings are relevant for speech and language therapies within this patient group, deserving further investigation.

\section{Acknowledgments}

We kindly acknowledge the Dutch Galactosemia Patient Association (GVN) for their cooperation and assistance during the preparation of this study, and the participants and their parents for their time, effort and cooperation. We thank Francesco Gentile for the preparation of the experimental stimuli and support with the analyses. 

CHAPTER 4

\section{THE FUNCTIONAL NEURAL NETWORK OF SENTENCE PRODUCTION DELINATES INTO SYNTACTIC AND MOTOR SPEECH PLANNING NETWORKS: AN FMRI CONNECTIVITY STUDY}

Inge Timmers, Job van den Hurk, M Estela Rubio-Gozalbo, and Bernadette M Jansma (in preparation). The functional neural network of sentence production delineates into syntactic and motor speech planning networks: an fMRI connectivity study 


\section{Summary}

During syntactic sentence planning, we retrieve and assign syntactic information, and assemble words and phrases into sentences. In the present study, we investigated the neural network involved in overt sentence production. By using functional magnetic resonance imaging (fMRI) and studying both activation and connectivity patterns, we aimed to learn how the language production network delineates into networks associated with syntactic planning and motor speech planning. Participants were instructed to overtly describe animated visual scenes using one of three naming formats, varying in syntactic complexity (from single words, to noun phrases, to a sentence). First, we examined which brain areas showed a parametric modulation with syntactic complexity. Both the left inferior frontal gyrus (IFG) and left pre-supplementary motor area (pre-SMA) showed this modulation: activity increased with increasing syntactic complexity. Then, these two regions were taken as seeds in a seed-based functional connectivity analysis to investigate with which brain areas these syntactically-modulated areas functionally connect. Both seed regions correlated with different functional networks: the left IFG with inferior and middle frontal areas, insula, superior temporal sulcus / middle temporal gyrus and supramarginal gyrus - a left-lateralized network associated with syntactic processing. The left pre-SMA in turn correlated with bilateral (pre)SMA, precentral gyus, superior temporal gyrus/planum temporale, and superior parietal regions - a bilateral network associated with motor speech planning. The present study shows that the language network has specialized functional sub-networks that may work in parallel to deal with different aspects of language production. Such delineation is necessary and useful for better understanding of the language production network and disorders that affect its functioning. We further demonstrate that overt naming can be studied in fMRI using animated scenes to make the production task as natural as possible. 


\section{Introduction}

Planning sentences is a universal part of everyday language production. Syntactic planning is required to retrieve and assign syntactic information, and to construct the phrases to make up the sentence. There is still debate on the specifics of how we carry out syntactic planning, but there is general agreement from psycholinguistic research that once the initial noun phrase plan is there, motor speech planning starts preparing the appropriate articulation (Bock, 1995; Levelt, 1989). Recent electrophysiological studies focusing on temporal aspects of syntactic complexity have found syntactic modulations around 300-500 ms after onset stimulus (Marek et al., 2007; Sahin et al., 2009; Timmers et al., 2013), supporting psycholinguistic models of speech production (Indefrey and Levelt, 2004). There is also evidence for continuing syntactic integration after the initiation phase, i.e., at later time points during sentence production (Timmers et al., 2013). Several PET and fMRI studies investigated regions of interest associated with syntactic planning. We will follow up on these studies to detect functional networks associated with syntactic processing during speech production.

From previous fundamental studies, we know that the left inferior frontal gyrus (IFG) plays an important role in syntactic processing (see reviews of e.g., Cappa, 2012; Price, 2012). Modulating the amount of syntactic planning in language production resulted in parametric variations in the left IFG, or more specifically the left Rolandic operculum (Indefrey et al., 2001). Recent adaptation studies also indicated a prominent role for this region in syntactic planning in sentence context (Menenti et al., 2011; Segaert et al., 2011), both for production and for comprehension. Direct electrophysiological measures in this area further revealed distinct activity for grammatical processing, but also for lexical and phonological processing (Sahin et al., 2009). This latter finding illustrates the notion that the left IFG has been associated with more than syntactic processing only. In interactive frameworks, it is suggested that the left IFG plays an important role in unification: retrieving relevant words from the mental lexicon (long term memory) and combining them into larger units (Hagoort, 2005, Vosse and Kempen, 2000). In comprehension, studies have found that the left posterior IFG is the site where the online integration or unification takes place after the relevant lexical-syntactic information has been recruited, in which the posterior middle temporal gyrus (MTG) plays a relevant role (Snijders et al., 2009; 2010). A similar interaction of lexical-syntactic retrieval and syntactic unification might take place during production. In addition, the left IFG - or more specifically the pars opercularis - is also associated with 
motor response selection, in a network also including pre-SMA (BA 6), cingulate motor area, and ventral premotor area (Tremblay and Small, 2011).

Although the left IFG is an important region of interest, it is not the only one. Recently, a study investigated syntactic processing in a natural setting (story listening), and found involvement of the left anterior temporal lobe (aTL) in syntactic computations (sentence structure building) (Brennan et al., 2012). The authors suggested that left IFG was involved in more memory-related syntactic operations, while the aTL was associated with structure building computations. Other studies have found a collection of regions also showing modulations with syntactic processing including left posterior middle temporal gyrus (MTG, BA 21), and bilateral (pre)supplementary motor areas (pre-SMA; BA 6) (Menenti et al., 2011; Segaert et al., 2011). In a sentence generation paradigm, the left medial frontal gyrus (BA 6), superior parietal lobule (BA 7), and right insula (BA 13) have been implicated in syntactic processing as well (Haller et al., 2005). In another study attempting to separate confounding influences from working memory and articulation in syntactic processing, a similar language network was found to be recruited during grammatical processing, including left IFG (BA 44/45/47), anterior insula, and medial SMA (Sahin et al., 2006). The authors suggested that BA 45 and the insula were associated more with the articulation aspects instead of pure syntactic processing.

Although several brain areas have been implicated in syntactic planning, there have been no studies yet directly investigating sentence production in a functional network context. We employed a paradigm in which syntactic complexity is varied in a relatively natural manner (adapted from Indefrey et al., 2001; see also Timmers et al., 2012). Participants were instructed to overtly describe a visually animated scene using response conditions that varied in their syntactic planning requirements. To control for visual processing demands related to the complex stimuli, passive watching conditions were added in which identical stimulation was presented without any language component. In this explorative study, we first identified which regions showed a parametric modulation with syntactic complexity. Then, we used a seed-based functional connectivity analysis to investigate with which areas these syntacticallymodulated regions functionally correlated. A second aim was to disentangle networks engaged in syntactic planning and motor speech planning. This aim is of relevance from a fundamental point of view on the integrative nature of language processing. However, it is also of interest from the perspective of diagnostic aspects in language disorders, as disorders may result from problems in one of the relevant networks, or from impairments in both the syntactic planning and motor speech planning network. 


\section{Methods}

\section{Participants}

Twelve healthy volunteers participated in this study. Ten were female and two were male, having a mean age of 24.4 years (SD 2.0 years). All participants were right-handed, had normal or corrected to normal vision, and were native Dutch speakers. They received financial compensation or received academic credit points for participating in the study. Participants were screened for MRI compatibility and signed informed consent. The ethical committee of the Faculty of Psychology and Neuroscience, Maastricht University, gave clearance for the study.

\section{Language paradigm}

Visually animated scenes were presented to the participants, consisting of three coloured geometric figures of which one moved towards another figure in one of two different ways (either 'to fly towards' or 'to bump into'). Content of the scenes was randomized. They were instructed to either passively watch the scene (which could be static or animated, referred to as 'passive watching static' ['PS'] or 'passive watching animated' ['PA'], respectively) or describe the scene using one of three overt responses that varied in syntactic complexity. More specifically, participants were asked to either use a complete sentence (e.g., "The red triangle bumps into the green square.", sentence-level syntactic planning; referred to as the 'sentence' condition or ' $S$ '), to use a noun phrases (e.g., "the red triangle", "the green square", "to bump into", noun-phrase level syntactic planning; referred to as the 'noun phrase' condition or 'NP') or to use separate words (e.g., "triangle", "red", "square", "green", "to bump into", minimal syntactic planning; referred to as the 'word' condition or 'W'). For more detailed information on the paradigm, see Timmers et al. (2012; 2013).

\section{Procedure}

The sessions started with providing the participants with information on scanning procedures, and safety measures. Participants received instructions and practiced the language task (the practice version consisted of 12 practice trials per condition). After safety precautions were taken (e.g., removal of any metals, ear plugs in order to damp the sounds), participants were placed comfortably inside the scanner, with their head fixed with soft foam pads. Through a mirror attached to the head coil, participants were able to see a screen on which the stimuli were projected. 
During the acquisition of the functional images, participants performed the language task, consisting of 4 runs in a blocked design (see Figure 4.1). In each run, there were 10 blocks (two per condition), and per block there were 5 trials of scenes. Per set of five blocks (each condition occurring once), conditions were randomized. Each run started with a repetition of the general instructions of the task. Prior to each block, there was a baseline period of 5 TR (10 s) during which a fixation point was displayed, followed by the presentation of the instruction for the specific block for 2 TR $(4 \mathrm{~s})$. The instruction included an acronym for the required naming format accompanied by an example in case of the overt descriptions, followed by a baseline period of 3 TR ( $6 \mathrm{~s})$. Trials started with a fixation period of $1 \mathrm{TR}(2 \mathrm{~s})$, followed by the onset of the scene (animated plus freeze period; total duration of 4.5 seconds) and a blank screen (1.5 seconds) (together $3 \mathrm{TR}$ ). From the onset of the scene, participants had 6 seconds to give their response. The total trial duration was 4 TR $(8 \mathrm{~s})$, and the total block duration was 60 seconds. One run had a duration of approximately 10 minutes. Per participant, fMRI data of a total of 40 blocks were recorded (8 per condition). Participants were instructed not to move their heads, and to speak at a soft volume to minimize movement. They were asked to describe the scenes as fast and as accurate as possible and to abort the descriptions in case the fixation mark signalled the subsequent scene (to avoid time pressure). Stimulus presentation was synchronized with MR data acquisition using Presentation Software (Neurobehavioral Systems Inc).

\section{Data acquisition}

Data were acquired on a 3-T Siemens Magnetom Allegra head scanner (Siemens Medical System, Erlangen, Germany) using a 8-channel head coil, and a 3-T Siemens Trio whole body scanner (Siemens Medical System, Erlangen, Germany), using a 32-channel head coil (acquisition on two different scanners was necessary because of irresolvable technical problems with the first scanner and head coil; acquisition parameters were identical unless specified otherwise).

For the functional MRI sequence, thirty-two axial slices $(3.5 \mathrm{~mm}$ iso-voxel, interslice distance $0 \mathrm{~mm}$ ) covering the entire cortical volume were collected using a standard echo-planar imaging (EPI) sequence (repetition time $[T R]=$ $2000 \mathrm{~ms}$, matrix size $=64 \times 64$, echo time $[\mathrm{TE}]=30 \mathrm{~ms}$, flip angle $=90^{\circ}$ ). For each run, we collected 305 functional volumes, of which the first 4 were excluded from subsequent data analysis due to T1 saturation. 


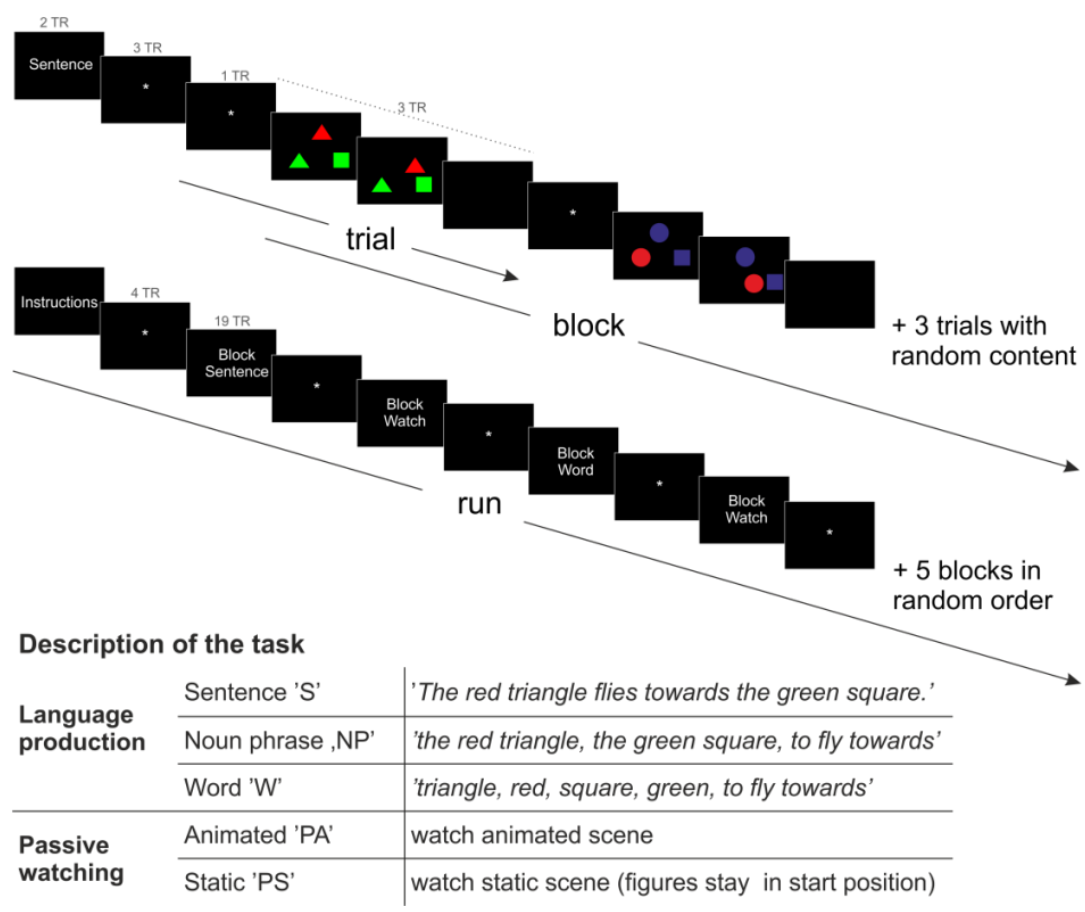

Figure 4.1. Schematic overview of the language production task. Showed are the contents of one block (top: starting with an instruction of the specific block condition, followed by five trials of the same condition, comprising one block) and one run (bottom: a run starts with general instructions of the task, followed by eight blocks - two per condition - preceded by a fixation point).

In order to be able to post hoc correct the EPI data for inhomogeneities in the magnetic field, a field mapping sequence was acquired $(T R=704 \mathrm{~ms}$; $T E[1]=$ $5.11 \mathrm{~ms} ; \mathrm{TE}[2]=7.57 \mathrm{~ms}$, identical slice positioning to EPI acquisition).

Anatomical images were obtained using a $1 \times 1 \times 1 \mathrm{~mm}$ resolution T1-weighted ADNI MPRAGE sequence $\left(\mathrm{TR}=2250 \mathrm{~ms}\right.$; $\mathrm{TE}=2.6 \mathrm{~ms}$; flip angle $\left.=9^{\circ}\right) .192$ slices were collected covering the entire brain.

\section{Data analysis}

Data were analysed using the BrainVoyager QX 2.6 software package (Brain Innovation, Maastricht, the Netherlands). Functional data were first corrected for EPI distortions using the anatabacus plugin for BVQX (Breman et al., 2009), which applies the pixelshift algorithm (Jezzard and Balaban, 1995). The undistorted data were pre-processed: slice scan time differences and 3D head motion were corrected (all runs were aligned to the third run - closest in time to the anatomical scan - in order to improve co-registration). Linear trends were 
removed and a temporal high pass filter was applied (4 cycles per run, or 1 cycle per 75 volumes). After pre-processing, the functional runs were coregistered with the $3 \mathrm{D}$ anatomical data and normalized to Talairach space.

By segmenting the grey and white matter in the anatomical images, white matter reconstructions of both hemispheres of each participant were made. Per hemisphere, cortical meshes were aligned to a dynamic group average using a cortex based alignment algorithm (Goebel et al., 2006). A group-aligned average cortical surface of each hemisphere was created. On the merged cortical surface, the univariate random-effects (RFX) statistical analysis was performed. A design matrix was created with the 5 conditions predictors (i.e., 'S', 'NP', 'W', 'PA', 'PS'), adjusted for the hemodynamic response delay. The six parameters describing the $3 \mathrm{D}$ head motion ( 3 translation parameters, 3 rotation parameters) were normalized and added as confound variables. In addition, the mean signal from the cerebral spinal fluid (CSF) and white matter (WM) was extracted, normalized and added as confounders as well. Global statistical contrasts between the three language conditions and passive watching I baseline were inspected at the level of the whole cortex, FDR corrected at $q=$ .05. Subsequently, patches of interest (POIs) were extracted, of which the beta values per language condition were extracted. The beta values were fed into a repeated measures General Linear Model in order to evaluate syntactic complexity effects. Based on the syntactic complexity effects, POls were selected as seeds for a seed-based functional connectivity analysis. In this analysis, the entire time course of a specific region of interest or seed is extracted and correlated with all other vertices on the cortical surface. Regions that showed spatiotemporal synchronicity with the seed region were subsequently interpreted as forming a functional network with the seed region (Huettel et al., 2004; Li et al., 2009). For the seeds, the peak vertex of the POI was determined and dilated to 61 vertices. The time course of the POI was extracted, normalized and fed into an RFX analysis as predictor, together with the above-specified confound variables. Resulting maps were inspected at the level of the whole cortex, FDR corrected at $q=.05$.

\section{Results}

\section{Cortical surface analysis}

A contrast analysis of the three language conditions with baseline (' $\mathrm{S}$ ' + 'NP' + 'W' > baseline) revealed several regions, including occipital regions (e.g., motion area, MT), left IFG, bilateral (pre)motor and STG / planum temporale (PT), left pre-SMA and the superior parietal region (see Figure 4.2A). 
To exclude confounding by visual processing, we contrasted the three language production conditions with the two passive watching conditions ('S' + 'NP' + 'W' $>$ 'PA' + 'PS'). A similar network of regions appeared, including bilateral (pre)motor and STG, left pre-SMA, left IFG (pars opercularis) and the right insula (depicted in Figure 4.2B). Controlling for visual confounding was confirmed by the absence of occipital regions in this contrast (visual inspection).
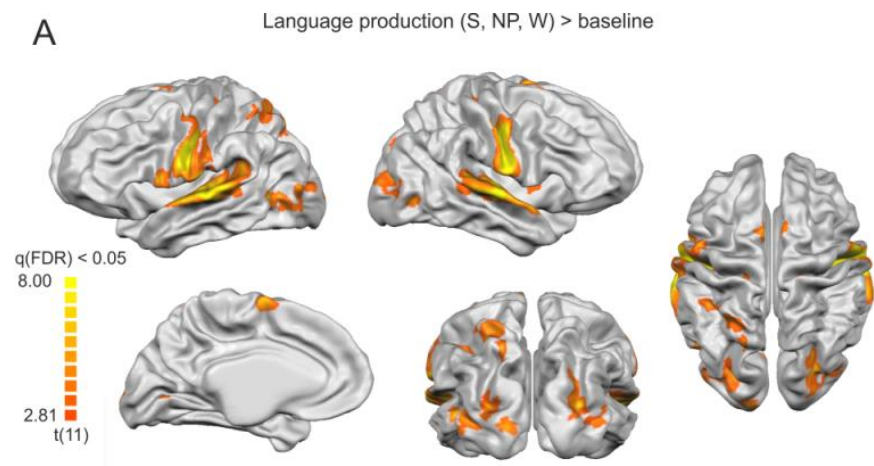

B Language production $(\mathrm{S}, \mathrm{NP}, \mathrm{W})>$ passive watching $(\mathrm{PA}, \mathrm{PS})$
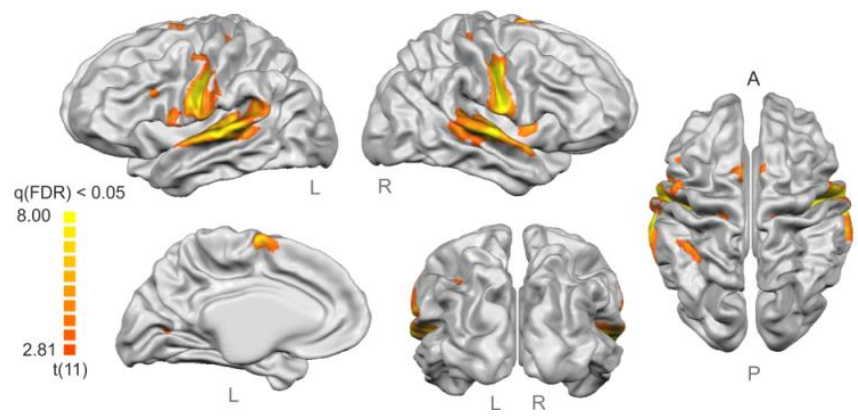

Figure 4.2. RFX based contrast analysis of overt sentence processing. A. Areas that are activated more during language production compared to baseline. B. Brain regions activated more during language production than during passive watching of similar animated visual scenes. Both statistical contrast maps are FDR corrected at $q=.05$, with a cluster size threshold of $25 \mathrm{~mm}^{2}$.

\section{Patches of interest analysis}

From the language-specific contrast ('S' + 'NP' + 'W' > 'PA' + 'PS'), patches of interest (POls) were extracted (cluster size threshold $=50 \mathrm{~mm}^{2}$ ). Ten POls survived the threshold: left and right superior temporal area / PT, left and right ventral precentral gyrus/central sulcus, left and right dorsal central sulcus, left (pre)SMA, left IFG, right insula and two regions within the left superior parietal 
cortex. Beta values were extracted in order to inspect for language-specific condition effects.

Table 4.1 presents those POls in which a syntactic effect was observed. A linear contrast was found in both the left (pre)SMA and the left IFG: beta values were higher for ' $S$ ' than for 'NP' than for 'W'. Both left and right ventral precentral areas showed a significant syntactic modulation effect as well, explained by a quadratic contrast.

Table 4.1. Patch of interest analysis: regions showing a significant modulation with syntactic complexity

\begin{tabular}{|c|c|c|c|c|c|c|c|c|}
\hline \multirow[b]{2}{*}{ Region } & \multirow[b]{2}{*}{ contrast } & \multirow[b]{2}{*}{$F$} & \multirow[b]{2}{*}{$p$} & \multirow[b]{2}{*}{ modulation } & \multirow[b]{2}{*}{ \# vertices } & \multicolumn{3}{|c|}{ peak coordinates } \\
\hline & & & & & & tal $x$ & tal y & tal z \\
\hline \multicolumn{9}{|l|}{ Left } \\
\hline inferior frontal gyrus & linear & 5.14 & 0.045 & $S>N P>W$ & 103 & -51 & 8 & 14 \\
\hline ventral precentral area & quadratic & 11.66 & 0.006 & $\mathrm{NP}>\mathrm{S}, \mathrm{W}$ & 1279 & -50 & -11 & 26 \\
\hline $\begin{array}{l}\text { (pre)supplementary } \\
\text { motor area }\end{array}$ & linear & 12.51 & 0.005 & $S>N P>W$ & 134 & -5 & -6 & 62 \\
\hline Right & & & & & & & & \\
\hline ventral precentral area & quadratic & 5.199 & 0.044 & $S, N P>W$ & 956 & 39 & -15 & 33 \\
\hline
\end{tabular}

\section{Seed-based correlation analysis}

The dilated peak vertices of the POls of the two areas showing a linear relation with syntactic complexity (i.e., left IFG and left pre-SMA) were placed as seeds in two separate seed-based correlation analyses. Resulting connectivity maps, plus the corresponding seeds are presented in Figure 4.3. When placing the left IFG as a seed, activation correlated with that of several regions, including left insula, left inferior and middle frontal regions (to lesser extent on right), middle temporal gyrus (MTG) / superior temporal sulcus (STS), supramarginal gyrus (SMG), and medial superior frontal region (anterior cingulate region). Using the left (pre)SMA as seed, we observed again a correlation with several regions, including bilateral (pre)supplementary motor regions, precentral gyrus and sulcus, bilateral superior parietal regions, and bilateral STG / PT. 

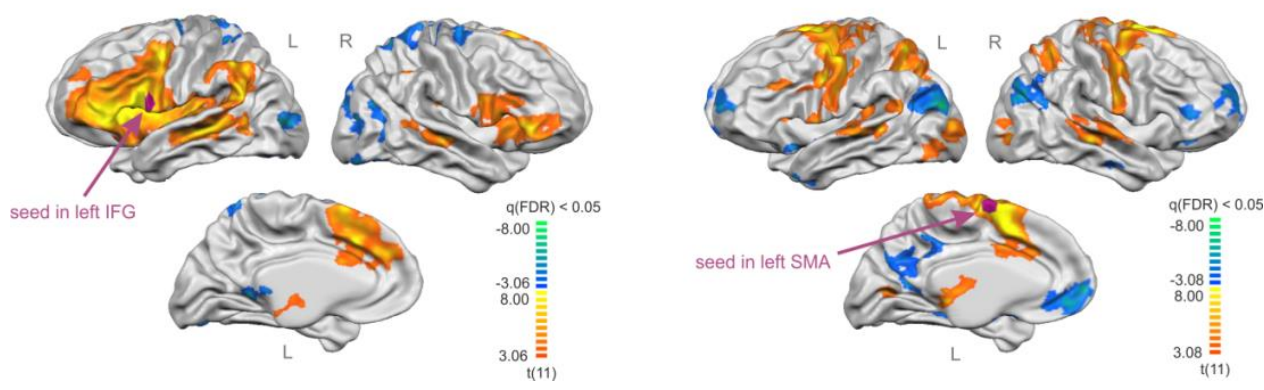

Figure 4.3. Correlation maps of two separate language networks based on seed-based correlation analysis. Left: left IFG as seed; right: left SMA as seed. Both contrasts are based on an RFX analysis, statistical map FDR corrected at $q=.05$, cluster size threshold of $25 \mathrm{~mm}^{2}$. Seeds were defined based on their syntactic sensitivity, as determined by a POI analysis.

\section{Discussion}

The current study aimed to investigate the neural network involved in sentence production, and to see how it delineates into networks associated with syntactic planning and motor speech planning. We used a paradigm in which the amount of syntactic planning was parametrically varied from words to noun phrases to sentences (Indefrey et al., 2001; see also Timmers et al., 2013). Visual processing of the complex animated visual stimuli was controlled for by contrasting the language production conditions with passive watching conditions using identical scenes without the language component. An explorative analysis approach was chosen in which we first examined which brain areas showed a parametric modulation with syntactic complexity. It appeared that both the left IFG and left pre-SMA showed this modulation: activity increased with increasing syntactic complexity. Then, these two regions were taken as seeds in a seedbased functional connectivity analysis, to investigate with which brain areas these syntactic-modulated areas functionally correlate or communicate. Two networks of regions appeared, which will be discussed in more detail below.

The finding that left IFG and left pre-SMA modulated with syntactic planning supports previous findings. Several studies have observed such modulations within these regions (Haller et al., 2005; Indefrey et al., 2001; Menenti et al., 2011; Segaert et al., 2011). The left IFG is suggested to be involved in unification or integration of syntactic processes (Hagoort, 2005; Snijders et al., 2010; Vosse and Kempen, 2000). Consistent with an integration role, the observed correlation of IFG with middle frontal gyrus (MFG) or dorsolateral prefrontal frontal cortex could reflect cognitive control and working memory 
interactions. The correlation with superior medial frontal or anterior cingulate regions may reflect performance monitoring or response suppression processes (see Wood and Grafman, 2003, for an overview on prefrontal cortex; Price, 2012). Correlations of IFG with the left insula, superior temporal sulcus (STS) / middle temporal gyrus (MTG), and supramarginal gyrus (SMG) relate to language production (note that the observed regions show great overlap with a resting state functional connectivity study using, among other regions, the left IFG/pars opercularis as a seed, Xiang et al., 2010). The posterior MTG has been suggested to be a lexical interface, linking semantic information to other relevant information (Hickok and Poeppel, 2007). In sentence comprehension, the posterior MTG was suggested to be the site where lexical and syntactic properties are retrieved from the mental lexicon (Snijders et al., 2009, 2010). The STS in its turn has been related to phonological planning, and is suggested to be the site for phonological processes and representations (Hickok, 2009). It is plausible that in analogue to the interface between left IFG and posterior MTG for lexical-syntactic information, the STS is important for retrieving phonological information, that will be integrated later in left IFG. Further, the SMG has been implicated in phonological processing as well (Price, 2010; 2012), while the left anterior insula is described as part of the articulatory network (Hickok and Poeppel, 2007; Price, 2010; 2012). Through its connections with these regions, the left IFG indeed seems to be involved in the syntactic processing (varying across the naming conditions), and can well be associated with 'integration' or 'unifying' during sentence production.

The pre-SMA is part of the so called supplementary motor complex (SMC), which consists of SMA proper, pre-SMA and the supplementary eye field (Nachev et al., 2008). Pre-SMA lies anterior to the vertical anterior commissure line, while SMA lies posterior to it. In our study, the syntactic modulation was observed in pre-SMA, although the distinction is only a fine one. Studies have shown that the SMC makes an important contribution to (learning of) the sequencing of actions. Pre-SMA is activated in tasks requiring some form of cognitive control, or inhibiting or switching between tasks or responses (see Nachev et al., 2008, for a review). Segaert et al. suggested to interpret the observed syntactic modulations in pre-SMA in terms of the sequencing of syllable structures (2011). Indeed, during sentence production and in the current paradigm, switching and inhibiting responses, as well as sequencing plays an important role (i.e., sequencing of syllables, but also of words and phrases). It could be speculated that sequencing differs across naming conditions. The sentence ('S') condition might trigger more extensive sequencing activity within pre-SMA, compared to artificial telegram style listing separate words (as is the case in the words ' $W$ ' condition). In terms of functional connectivity, the activity 
in pre-SMA was correlated with bilateral STG / PT, SMC, (pre)motor regions in precentral gyrus and sulcus, and superior parietal cortex. A comprehensive review associates the pre-SMA, SMA and motor cortex in the initiation and execution of speech (Price, 2010, but see also e.g., Bouchard et al., 2013; Indefrey and Levelt, 2004), while the superior parietal region and PT are implicated in sensory-motor integration processes (Hickok and Poeppel, 2007). The STG has a relevant role in phonological planning and monitoring (Indefrey and Levelt, 2004; Christoffels et al., 2007; 2011). Thus, compared with the left IFG-syntax network, this pre-SMA seed-based network seems to be much more motor speech related.

It should be noted that although the seed-based functional connectivity analysis provides statistically straightforward data, it has some important limitations (see also Li et al., 2009). One drawback is that the correlations are calculated across the entire time course. This means that the presented connectivity maps might not be purely related to language production activity, but also to the passive watching conditions or even to the baseline (rest) periods. Vice versa, it might be the case that the connectivity maps are driven primarily by one of the conditions (e.g., the sentence condition). Future analyses could be directed towards selecting the activity specific for the conditions. This would allow to examine modulations in the connectivity patterns per condition (i.e., as in the psychophysiological interaction approach, Friston et al., 1997). In addition, obtained results give information concerning correlations, but not concerning the direction of influences. Methods such as Granger Causality could be used to investigate effective connectivity patterns, and learn which areas in the obtained maps influence which (Roebroeck et al., 2005).

To conclude, in this explorative study, fMRI contrasts revealed that both left IFG and left pre-SMA show parametric modulation with complexity during syntactic planning of overt speech. The functional connectivity analysis with left IFG and left pre-SMA as seeds, most interestingly revealed that both areas are operating within different neural circuits within the language network. Left IFG is functionally connected within a left lateralized syntactic-integration network, and left pre-SMA with a bilateral motor speech network. Overall, this study demonstrated that overt naming can be well studied in fMRI using animated scenes, to make the production task as natural as possible. It furthermore showed that different neural circuits within the language network can be delineated within the same cognitive task. 
<smiles>CCCCCCC</smiles> 
CHAPTER 5

\section{NEURAL NETWORKS RELATED TO SYNTACTIC AND MOTOR SPEECH PLANNING ARE AFFECTED IN CLASSIC GALACTOSEMIA}

Inge Timmers, Job van den Hurk, Paul AM Hofman, Luc JI Zimmermann, Kamil Uludağ, Bernadette M Jansma, and M Estela Rubio-Gozalbo (submitted). Neural networks related to syntactic and motor speech planning are affected in classic galactosemia. 


\section{Summary}

Patients with the inherited metabolic disorder classic galactosemia have language production impairments in several planning stages. The characteristics of these impairments are not yet understood. Here, we focus on the syntactic aspect of sentence planning. We used functional magnetic resonance imaging (fMRI) to study neural activity and connectivity while the patients carried out an active language production task. Participants were asked to describe an animated visual scene using one of two language conditions, varying in syntactic complexity (single words versus a sentence). In cognitive terms, this task involved visual processing, conceptualisation of the target objects and action, followed by syntactic and phonological planning, and articulation. Results showed that the patients recruited a generally similar network of brain regions compared to healthy controls, but recruited additional and more extensive regions. Both groups showed modulations with syntactic complexity in left inferior frontal gyrus (IFG), a region associated with syntactic planning, and in right insula - mainly active in patient group. In addition, patients showed a modulation with syntax in left superior temporal gyrus (STG), whereas the controls did not. Further, patients showed increased activity in right STG and right supplementary motor area (SMA). In the functional connectivity patterns, a similar pattern emerged with more extensive connectivity with frontal and motor regions, and more restricted and weaker connectivity with superior temporal regions. Patients also showed higher baseline cerebral blood flow (CBF) in right IFG and trends towards higher CBF in bilateral STG, SMA and the insula. Taken together, the data demonstrate that language abnormalities in classic galactosemia are associated with specific changes within the language network. These changes point towards impairments related to both syntactic planning and motor speech planning in patients with classic galactosemia. 


\section{Introduction}

There is neuroscientific evidence for language production impairments in several planning stages in patients with classic galactosemia, an inborn error of galactose metabolism (Timmers et al., 2012). In the current study, we used functional magnetic resonance imaging (fMRI) to investigate potential deviations in functional neural networks involved in language production.

Classic galactosemia is a potentially lethal disorder that results from a profound deficiency of galactose-1-phosphate uridyl transferase (GALT) enzyme activity, involved in the metabolism of galactose important for energy delivery and glycosylation of complex molecules (Holton et al., 2001). Most infants with galactosemia are born apparently healthy, but after exposure to breast milk or a milk-based formula (containing large amounts of galactose), suffer a rapid and devastating demise. Early diagnosis dietary restriction of galactose, prevents or resolves the acute manifestations of the disease. However, significant complications appear later in childhood. Patients may experience complications related to the bones, the ovaries and the brain (Panis et al., 2004; RubioGozalbo et al., 2010; Waggoner et al., 1990; Waisbren et al., 2012). Neurological and cognitive impairments include a lower intelligence, memory impairments, slower information processing, and motor dysfunction (Antshel et al., 2004; Doyle et al., 2010; Rubio-Agusti et al., 2013; Widhalm et al., 2002). Classic galactosemia is also associated with voice disorders, motor (speech), and language impairments. In her study, Potter (2011) found that about $58 \%$ of the children with galactosemia had decreased respiratory-phonatory support for speech, and disturbed vocal quality was observed in $33 \%$ of the patients. The author suggested that the observed problems were indicative of cerebellar dysfunction. Childhood apraxia of speech (CAS) or verbal dyspraxia has traditionally been reported as an explanation for the speech- and language impairments in galactosemia (Nelson et al., 1991; Robertson et al., 2000; Waggoner et al., 1990), although recent estimations indicate that only about 20$25 \%$ of the patients with galactosemia meet the criteria for CAS (Potter, 2011; Shriberg et al., 2011). In addition, it has been recognised that patients with galactosemia do not only have speech or motor related abnormalities (or both, Potter et al., 2013), but also impairments in language planning. Early reports already described decreased vocabulary, grammar and word retrieval problems (Schweitzer et al., 1993; Waggoner et al., 1990; Waisbren et al., 1983). Potter et al. (2008) showed that the majority of patients with galactosemia and a history of speech sound disorders also had language disorders, which could not be explained by lower cognitive abilities. A recent study showed a case with classic galactosemia who already at a pre-linguistic level (age 13 month) showed 
clinically significant delays on pre-linguistic skills (Lewis et al., 2013). The same group showed that three out of the four children in their study did not meet ageappropriate phonological awareness (aged 7-9) (Lewis et al., 2012). Recently, we used a high temporal resolution method, electroencephalography/event related potential (EEG/ERP), to investigate time windows sensitive to syntactic planning within patients and matched controls (Timmers et al., 2012). As $E E G / E R P$ is rather poor in spatial resolution, the current study used $\mathrm{FMRI}$ to compare the neural network associated with language production across patients and matched controls.

Previous brain studies in classic galactosemia have shown abnormalities. Nelson et al (1992) studied the brains of 67 treated patients (ranging in age from one month to 42 years) anatomically and found evidence of mild cerebral atrophy (often surrounding the lateral ventricles), cerebellar atrophy in some patients, and white matter abnormalities (hyperintensities on T2-weighted data). They postulated that the latter might reflect altered myelination as a result of abnormal galactocerebrosides which have been shown to be altered in histopathological studies in classic galactosemia (Haberland et al., 1971; Ng et al., 1989). Other studies have shown similar white matter changes in patients with galactosemia, histopathologically or with brain imaging (Crome, 1962; Hughes et al., 2009; Krabbi et al., 2011; Rubio-Agusti et al., 2013; Wang et al., 2001). In a $\left[{ }^{18} \mathrm{~F}\right]$ fluorodeoxyglucose (FDG) positron emission tomography (PET) study, Dubroff et al. (2008) found widespread decreases in cortical glucose metabolism (e.g., in the superior temporal gyrus [STG], medial occipital lobe, parietal lobe, cerebellum, calcarine cortex, superior frontal cortex, and superior parietal cortex) in addition to some increases in glucose metabolism (in the cingulate cortex and temporal poles). Also, nonspecific decreases in metabolism were found in the white matter. The authors suggested that there might be a relation with the neuropsychological profile of the patients. Hitherto, no clear relationship has been found between impairments observed during imaging and the cognitive outcome (Kaufman et al., 1995). In the current study, we aimed to directly link online language performance to neural data (see Timmers et al., 2011, for more information on the framework and approach).

According to neurocognitive theories of language planning, sentence production requires multiple planning stages over time. Based on perceptual input or thoughts, a speaker first finds concepts that he/she wants to express in a message. These concepts are transferred into neural representation of words and sentences in a process that involves access to meaning, syntactic function, and phonology. Once parts of this sentence plan are ready, the speaker starts to articulate. All this happens online within milliseconds, without us being aware 
of the underlying neural computations (Bock and Levelt, 1994; Levelt et al., 1999; Vosse and Kempen, 2000). In patients with galactosemia, high temporal resolution EEG/ERP showed that impairments occur already quite early in the process, starting from conceptualisation and continuing during lexical and syntactic planning (Timmers et al., 2012).

During language production, several brain regions are recruited, including areas in the superior temporal lobe, the temporal-parietal junction and in the inferior frontal lobe (Hickok, 2009; Indefrey, 2011, Price, 2010; 2012). For syntactic planning, the left inferior frontal gyrus (IFG) has been depicted as (one of) the most important regions (BA44/6/45) (see e.g., Cappa, 2012; Indefrey et al., $2001 ; 2004$ ), in addition to the medial frontal gyrus (BA 6), superior parietal lobule (BA 7), right insula (BA 13), left posterior middle temporal gyrus (MTG) and bilateral supplementary motor areas (SMA, BA 6) (Haller et al., 2005; Sahin et al., 2006; Menenti et al., 2011; Segaert et al., 2011). For lexical-semantic processing, the posterior MTG and anterior temporal lobe (ATL) have been implicated most (see Hickok, 2009). Conceptual planning is reflected in a network including left MTG, medial superior frontal gyrus (SFG), left MFG, and left angular gyrus/inferior parietal gyrus (Ye et al., 2011). The superior temporal gyrus (STG) in turn has been linked to phonological planning and monitoring (Indefrey and Levelt, 2004; Christoffels et al., 2007; 2011). The left posterior part of planum temporale (PT) (also called area Spt or Sylvian parietal-temporal area), has been associated with sensory-motor integration, because of evidence gathered in fMRI studies (Hickok et al., 2009), but also because of its connectivity to the motor areas (Buchsbaum et al., 2001).

The current study will characterize functional networks associated with active language production in patients with galactosemia as compared to controls. We acquired fMRI data to examine activity and connectivity patterns within relevant brain areas. An important issue in patient studies is a possible confounding role of abnormal cerebral blood flow (CBF) that can contribute to an abnormal fMRI signal (the blood oxygenation level dependent [BOLD] signal) (Uludağ et al., 2006). The BOLD signal is directly affected by the blood oxygenation and volume, and the blood oxygenation in turn changes with CBF, cerebral blood oxygen consumption (CMRO2), and cerebral blood volume (CBV). Baseline differences in CBF can have effects on the sensitivity of the BOLD signal, which could confound observed BOLD changes, which is why CBF is a relevant control for group comparisons in $\mathrm{fMRI}$. CBF was measured in this study using arterial spin labelling (ASL). In order to associate the high temporal resolution data to high spatial resolution data of the current study, we used a similar paradigm to the one used in the previous EEG study. Participants were 
instructed to either passively watch the presented visually animated scenes (control condition), or overtly describe it using one of two responses that vary in syntactic complexity (i.e., using sentences or separate words; see Indefrey et al., 2001; Timmers et al., 2012). We will examine whether there are any differences across groups as to which brain areas are recruited during language production, and more specifically syntactic planning, and as to which brain areas are functionally connected during this task.

\section{Methods}

\section{Participants}

Thirteen adolescent and young adult patients with galactosemia and thirteen healthy controls participated in this study. Classic galactosemia was diagnosed by GALT enzyme activity assay and/or GALT-gene mutational analysis. Two participants were excluded: one adolescent patient because of extensive motion during the scanning and one adolescent control because of a current health condition. Twelve patients remained in the analysis: three males and nine females, mean age 17.4 years (SD 1.9 years, range 14.6 - 21.1 years). Patient characteristics can be found in Table 5.1. The control group consisted of three males and nine females, mean age 17.1 years (SD 1.9 years, range $14.0-20.0$ years). Age did not differ significantly between the groups $\left[F_{1,22}=.12, p=.73\right]$. Participants had no other relevant health conditions. All had normal or corrected to normal vision, and were native Dutch speakers. All participants were screened for MRI compatibility and signed informed consent (in case of minors, both parents/caregivers also gave written informed consent). The Medical Ethical Committee of Maastricht University Hospital/Maastricht University (azM/UM) gave ethical clearance for this study.

Table 5.1. Classic galactosemia patient characteristics

\begin{tabular}{|rccc|} 
& N & $\begin{array}{c}\text { Mean / } \\
\text { Percentage }\end{array}$ & Range \\
\hline $\begin{array}{r}\text { Age at diagnosis (in days) } \\
\text { Age at introduction of diet (in days) }\end{array}$ & 12 & 11.1 & $0-35$ \\
Special education ${ }^{1}$ & 12 & $75 \%$ & $0-35$ \\
Speech therapy $^{1}$ & 12 & $92 \%$ & \\
Motor therapy $^{1}$ & 12 & $42 \%$ & \\
\hline
\end{tabular}




\begin{tabular}{|c|ccc} 
GALT activity (\% of reference value) & 12 & $0.55 \%$ & $0 \%-1.52 \%$ \\
\hline GALT mutation & 5 & $45 \%$ & Q188R / Q188R \\
& 1 & $9 \%$ & Q188R / L195P \\
& 3 & $27 \%$ & L195P / K229 \\
& 2 & $18 \%$ & other $^{2}$
\end{tabular}

${ }^{1}$ At some point in life; ${ }^{2}$ 400Tdel/unknown $(n=2)$

\section{Language paradigm}

Visually animated scenes, consisting of three coloured geometric figures, of which one moved towards another (either 'to fly towards' or 'to bump into'), were presented to the participants. They were instructed to either passively watch the scene or to describe it overtly. During passive watching (control condition), the scene could be static or animated, referred to as 'passive watching static' ['PS'] or 'passive watching animated' ['PA'], respectively. Further, during the main active naming conditions, the scene could be described using one of two overt responses that varied in syntactic complexity: a complete sentence (e.g., "The red triangle bumps into the green square.", sentence-level syntactic planning; referred to as 'overt description sentence' or ' $S$ ') or separate words (e.g., "triangle", "red", "square", "green", "to bump into", minimal syntactic planning; referred to as 'overt description words' or 'W'). Hence, in total there were four conditions. For more detailed information on the paradigm, see Timmers et al. (2012; 2013).

\section{Procedure}

The session started with acquainting the participants in a simulation (dummy) scanner (Maastricht University), where participants (and if present, their parents/caregivers) received information on the scanning procedures and safety measures. To prevent excessive motion during scanning, participants were explicitly instructed on the effects of motions, on how to prevent motion in the scanner and participants practiced lying and speaking in the scanner. After acquaintance, participants received instructions and practiced the language paradigm (the practice version consisted of 12 practice trials per condition, or more until adequate performance of the task was reached). After safety precautions were taken (e.g., removal of any metals, ear plugs in order to damp the sounds), the participant was placed comfortably inside the scanner, with their head fixed with soft foam pads. Through a mirror attached to the head coil, 
participants were able to see a screen on which the stimuli and an entertaining movie were projected (the movie already played while participants were placed in the scanner for distraction purposes).

Prior to the language task, the arterial spin labelling (ASL) sequence was recorded. During this scan, the participants were instructed to relax, clear their heads, and not think of anything in particular. A fixation point was presented, but participants were not obliged to fixate. It was not allowed, however, to close the eyes during this scan.

The main language task consisted of 4 runs in a blocked design. In each run, there were 8 blocks (two per condition), and per block there were 5 trials or scenes (see Figure 5.1). Conditions were randomized per set of four blocks (each condition occurring once). Prior to each block, there was a baseline period of 10 seconds (with a fixation point), after which the instruction for the specific block was presented for 4 seconds (accompanied by an example in case of the overt descriptions), followed by a baseline period of 6 seconds. Trials started with a fixation period of 2 seconds, followed by the onset of the scene (animated plus freeze period; total duration of 4.5 seconds) and a blank screen (1.5 seconds). From the onset of the scene on, participants had 6 seconds to give their response. Total trial duration was 8 seconds, and total block duration was 60 seconds. One run had a duration of approximately 8 minutes. Per participant, a total of 32 blocks were recorded (8 per condition). Participants were instructed to describe the scenes as fast and accurate as possible and to abort the descriptions in case the fixation mark signalling the next scene appeared (to avoid timing difficulties and stress). Stimulus presentation was synchronized with MR data acquisition by triggering the stimulus program (Presentation Software, Neurobehavioral Systems Inc) with the beginning of each trial.

\section{Data acquisition}

Data were acquired on a 3-T Siemens Magnetom Allegra head scanner (Siemens Medical System, Erlangen, Germany) using a 8-channel head coil, and a 3-T Siemens Trio whole body scanner (Siemens Medical System, Erlangen, Germany), using a 32-channel head coil (acquisition on two different scanners was necessary because of irresolvable technical problems with the first scanner and head coil; scanner parameters were identical unless otherwise specified; of each group four participants were scanned on the Allegra scanner). 


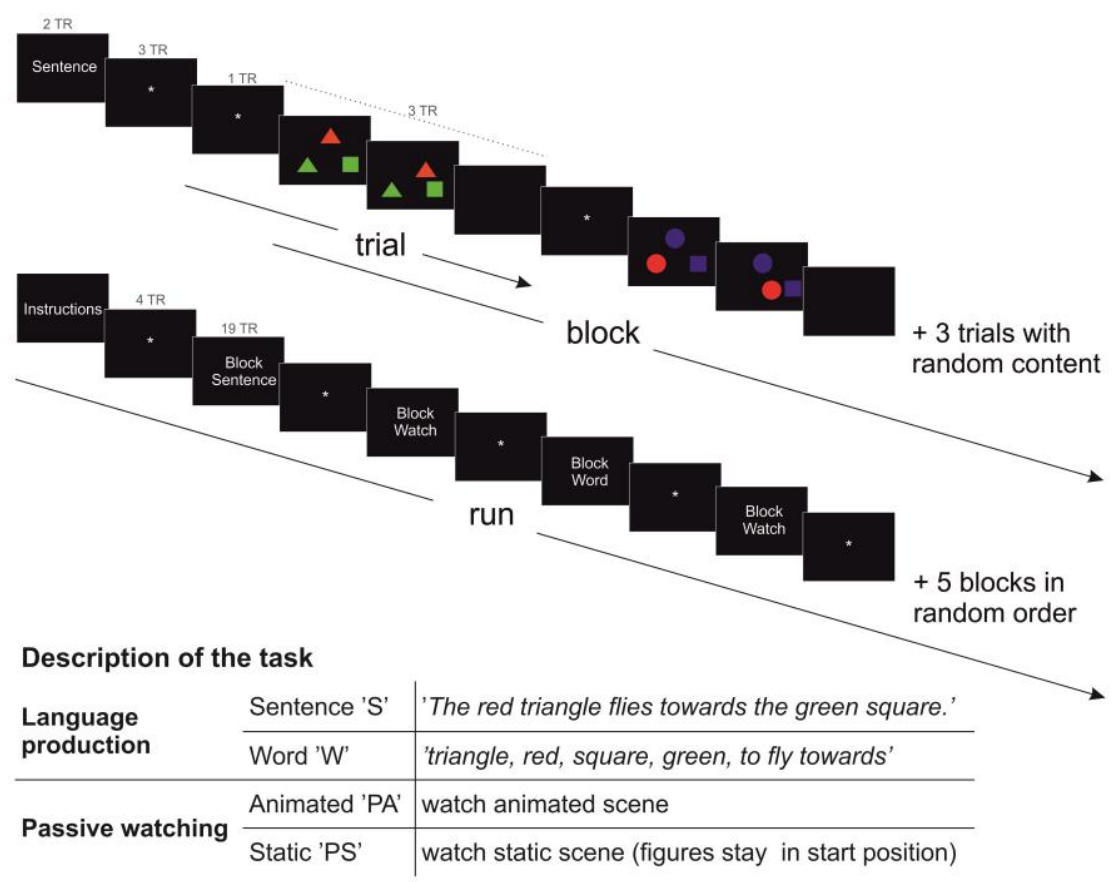

Figure 5.1. Schematic overview of the language production task. This figure shows the contents of one block (top: starting with an instruction of the specific block condition, followed by fixation points and five trials of the same condition, comprising one block) and one run (bottom: a run starts with general instructions of the task, followed by eight blocks -two per condition- preceded by a fixation point). Further, the conditions and instructed responses are described.

For the functional MRI sequence, thirty-two axial slices $(3.5 \mathrm{~mm}$ iso-voxel, interslice distance $0 \mathrm{~mm}$ ) covering the entire cortical volume were collected using a standard echo-planar imaging (EPI) sequence (repetition time $[\mathrm{TR}]=$ $2000 \mathrm{~ms}$, matrix size $=64 \times 64$, echo time $[\mathrm{TE}]=30 \mathrm{~ms}$, flip angle $=90^{\circ}$ ). For each run, we collected approximately 240 functional volumes, of which the first 4 were excluded due to T1 saturation.

For the functional MRI sequence, thirty-two axial slices $(3.5 \mathrm{~mm}$ iso-voxel, interslice distance $0 \mathrm{~mm}$ ) covering the entire cortical volume were collected using a standard echo-planar imaging (EPI) sequence (repetition time $[\mathrm{TR}]=$ $2000 \mathrm{~ms}$, matrix size $=64 \times 64$, echo time $[T E]=30 \mathrm{~ms}$, flip angle $=90^{\circ}$ ). For each run, we collected approximately 240 functional volumes, of which the first 4 were excluded due to T1 saturation. 
In order to be able to post hoc correct the EPI data for inhomogeneities in the magnetic field, a field mapping sequence was acquired $(\mathrm{TR}=704 \mathrm{~ms}$; $\mathrm{TE}[1]=$ $5.11 \mathrm{~ms} ; \mathrm{TE}[2]=7.57 \mathrm{~ms}$, identical slice positioning to EPI acquisition).

Anatomical images were obtained using a $1 \times 1 \times 1 \mathrm{~mm}$ resolution T1-weighted ADNI MPRAGE sequence (TR = $2250 \mathrm{~ms}$; TE = $2.6 \mathrm{~ms}$; flip angle $=9^{\circ}$ ). 192 slices were collected covering the whole brain.

For the ASL, we used the PICORE-QUIPSS II ASL sequence with the following parameters: $\mathrm{TI} 1=700 \mathrm{~ms}$, $\mathrm{Tl}=900 \mathrm{~ms}$ and $\mathrm{TI} 2=1400 \mathrm{~ms}$. 100 volumes were collected with 8 slices, positioned to cover the inferior frontal, superior temporal and inferior parietal regions $(T R=2000 \mathrm{~ms}$; TE $=20 \mathrm{~ms}$ [Trio: $\mathrm{TR}=17 \mathrm{~ms}$ ]; flip angle $=90^{\circ}$ ). In the calibration sequence, 10 volumes were acquired with identical slice positioning (TR $=10.000 \mathrm{~ms}$; TE $=20 \mathrm{~ms}$ [Trio: $\mathrm{TR}=17 \mathrm{~ms}$; ;lip angle $=90^{\circ}$ )

\section{Data analyses}

Data were analysed using the BrainVoyager QX 2.6 software package (Brain Innovation, Maastricht, the Netherlands). Functional data were first corrected for geometrical EPI distortions using the anatabacus plugin for BVQX (Breman et al., 2009), which applies the pixelshift algorithm (Jezzard and Balaban, 1995). The undistorted data were pre-processed: slice scan time differences and 3D head motion were corrected (all runs were aligned to the third run -closest in time to the anatomical scan- in order to improve co-registration). Runs in which the motion exceeded $10 \mathrm{~mm}$ were excluded from the analysis (i.e., 1 run was excluded); volumes in which the motion exceeded $5 \mathrm{~mm}$ were excluded from the design matrix (i.e., 8 runs of in total 4 participants -3 patients and 1 controlwere adapted). In general, however, the motion was less than the voxel size $(3.5 \mathrm{~mm})$. Linear trends were removed. After pre-processing, the functional runs were co-registered with the 3D anatomical data and normalized in Talairach space. Subsequently, a temporal high pass filter (3 cycles per run, or 1 cycle per approximately 80 volumes) and a 3D spatial smoothing procedure (Gaussian filter FWHM of $4 \mathrm{~mm}$ ) was applied.

By segmenting the grey and white matter in the anatomical images, white matter reconstructions of both hemispheres of each participant were made. Per hemisphere, cortical meshes were aligned to a dynamic group average using a cortex-based alignment algorithm implemented in BVQX (Goebel et al., 2006). A group-aligned average cortical surface of each hemisphere was created, after which the two hemispheres and corresponding data were merged again. 
Per participant and per run, a design matrix was created with the 4 conditions (i.e., S, W, PA, PS), adjusted for the hemodynamic response delay. The six parameters describing the 3D head motion (3 translation parameters, 3 rotation parameters) were normalized and added as variables of no interest (confounders), as well as the extracted mean signal from the cerebral spinal fluid (CSF) and white matter (WM) (as an estimate of physiological noise). On the cortical surface, a univariate random-effects (RFX) analysis was performed, per group. Resulting maps were overlaid and in overlapping regions (visually inspected), patches of interest (POIs; equivalent to region of interest [ROI] in surface space) were extracted (cluster size threshold $=25 \mathrm{~mm}^{2}$ ). The peak vertex was determined and dilated to 61 vertices, and beta values were extracted per condition and per participant. The beta values were fed into a repeated measures General Linear Model having age as a covariate in order to evaluate group and syntactic complexity effects. In addition, individual beta maps per condition were extracted from the RFX analysis in order to inspect group differences across the maps (based on t-test, p-values of 0.005 and lower are presented).

Further, based on syntactic complexity effects, POIs were selected as seeds for a seed-based functional connectivity analysis. In this analysis, the entire time course of a specific POI (i.e., seed) is extracted and correlated or regressed with all other vertices on the cortical surface. The rationale is that regions that show temporal correlations (or synchronisation) with the seed region are interpreted as forming a functional network with the seed region (Huettel et al., 2004; Li et al., 2009). Again, the dilated peak POls were used: the time course was extracted, normalized and fed into a RFX analysis as a predictor, together with the confound predictors (same as in univariate RFX; separate per group). In addition, individual beta maps per seed were extracted in order to examine group differences across the maps (based on t-test, p-value of 0.005 and lower are presented). Statistical contrasts were inspected at the level of the whole cortex, if not otherwise specified: FDR corrected at $q=.05$.

The perfusion-based arterial spin labelling (ASL) data were corrected for 3D head motion as well, after which they were co-registered to the anatomical data (both the ASL data and the calibration data). Using the volume-based ASL plugin in BVQX, absolute (quantitative) cerebral blood flow (aCBF) maps were created for each participant. CBF-weighted images were determined as the running difference of control and tagged images using the surround subtraction method, and calibration was done using the intensity M0 (see Çavuşoğlu et al., 2009 , for details). The resulting aCBF maps were converted to surface space and projected onto the cortical surface of each participant. The group-averaged 
dilated peak POls (i.e., all containing 61 vertices) were transferred back to individual subject space (i.e., as the aCBF maps were in subject space as well). From the individual POIs, the aCBF data were extracted. The data quality and efficiency of the tagging were evaluated by inspecting the aCBF values in the occipital cortex, and comparing the values to a reference value of around 50 $\mathrm{ml} / 100 \mathrm{~g} / \mathrm{min}$ for grey matter in visual cortex (Chen et al., 2008). Participants with absolute CBF values less than $20 \mathrm{ml} / 100 \mathrm{~g} / \mathrm{min}$ (to incorporate safety margin) were discarded from the analysis. In the final dataset, General Linear Model analyses were performed per $\mathrm{POI}$ in order to evaluate group differences in aCBF.

\section{Results}

The structural (T1-weighted) data of all patients were evaluated by a neuroradiologist $(P H)$. All were evaluated as normal, except one that showed evidence of cortical and subcortical atrophy.

\section{Language production sensitive areas}

Statistical maps of both groups displaying the regions that are sensitive to language production in comparison to baseline [' $S$ ' + ' $W$ ' > baseline] are shown in Figure 5.2A. A generally similar network of regions was observed in both groups, including the bilateral precentral gyrus (PG), bilateral superior temporal gyrus (STG) / planum temporale (PT), bilateral pre-supplementary motor area (pre-SMA), left inferior frontal gyrus (IFG), left insula, regions in the left superior parietal and in bilateral medial occipital lobe. Maps in Figure 5.2B show the contrast of passive watching versus baseline ['PA' + 'PS' > baseline]. It revealed that controls recruit several areas during passive watching that are also recruited during active language production, which is not/less present in patients. To compare the groups purely with respect to language production and to avoid confounding by differences in passive watching, all subsequent analyses will be restricted to the contrast language production versus baseline ['S' + 'W' > baseline] instead of to the contrast language production versus passive watching ['S' + 'W' > 'PA' + 'PS'].

Several group differences were observed. The patient group showed a more restricted involvement (i.e., less BOLD signal change) of the superior temporal lobe (bilateral) and the PT, and a more extensive recruitment in the left IFG, left $P G$ and bilateral pre-SMA. In addition, the right insula is recruited in the patients, whereas it is not statistically significant in the controls. 

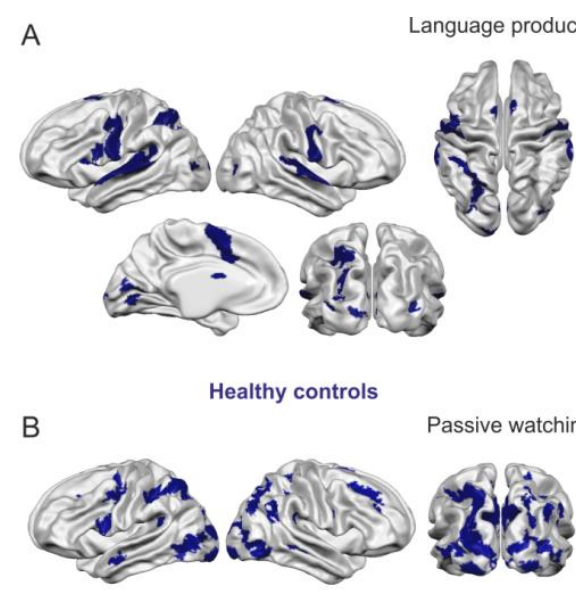

Passive watching versus baseline
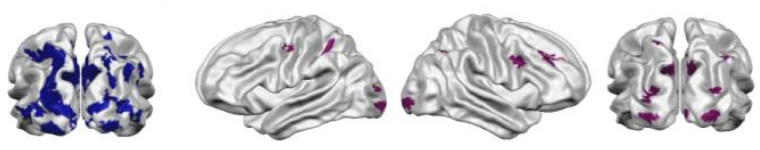

FDR $(q<0.05)$

Figure 5.2. Statistical maps (FDR correction threshold of $q<.05$ ), separate per group (controls displayed in dark blue; patients with galactosemia in purple). A. The contrast language production versus baseline ['S' + 'W' > baseline] is shown. $B$. The contrast passive watching versus baseline ['PA' + 'PS' > baseline]. For illustration purposes, the statistical threshold for this latter contrast was artificially lowered in the patients, to make it more comparable to the other condition and other group $(p<.005)$.

The statistical analysis corroborated that there are several regions showing a group difference in the BOLD response (see Figure 5.3). A lower BOLD signal change in the patient group as compared to the controls was found in left STG, left PT and bilateral occipital regions, while a higher signal change in the patients was observed in left middle and superior frontal regions, bilateral PG and right posterior insula.
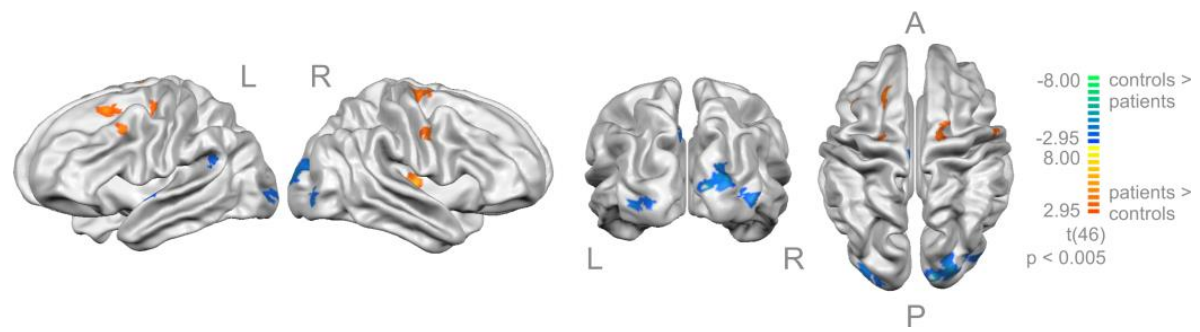

Figure 5.3. Group differences across the cortical surface in the contrast language production versus baseline ['S' + W' > baseline]. In red, areas are shown in which patients showed higher BOLD signal change compared to controls; in blue regions, the patients showed lower signal change compared to controls. 


\section{Patch of interest analysis}

In the POI analysis, several effects were found (see Table 5.2). A group effect was observed in the right STG and pre-SMA, where the patients showed higher BOLD signal change as compared to the controls. Further, there was an interaction effect between group and syntactic complexity in left STG: only the patients showed increased signal change during ' $S$ ' condition as compared to 'W'. In the left IFG and right insula, there is a main effect of syntactic complexity: sentences 'S' elicited higher signal change compared to words 'W' (no interaction effect; although right insula was only part of the patient's language network).

\section{Cerebral blood flow differences during baseline}

Three participants were excluded from the CBF analyses due to insufficient tagging efficiency as evaluated by the predetermined criterion (two patients, one control participant). The following POls were covered by the ASL sequence: bilateral IFG, STG and insula. Only the right IFG showed a significant difference across groups $\left[F_{1,16}=4.84, p=0.043\right]$, with patients having a higher aCBF compared to controls (see Figure 5.4).

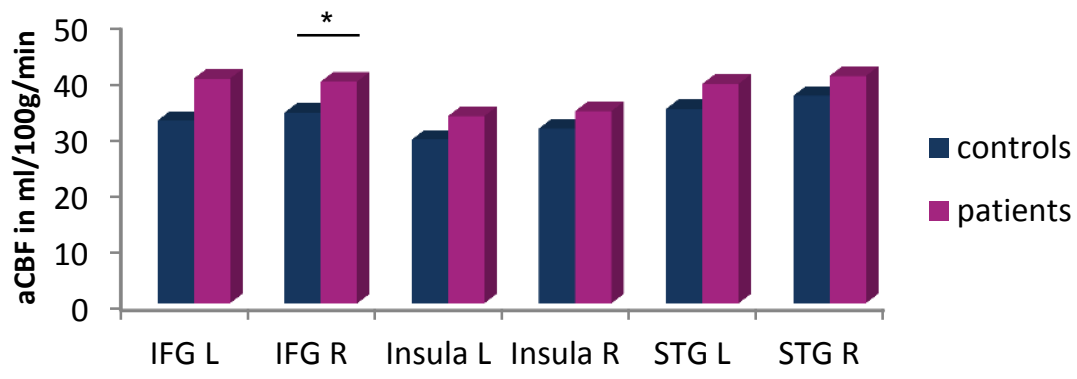

Figure 5.4. Overview of the baseline cerebral blood flow values, per group and per POI (all POls consisted of 61 vertices). Although a trend was present in all POls, the difference was significant only in right IFG. ${ }^{*} p<0.05$. 


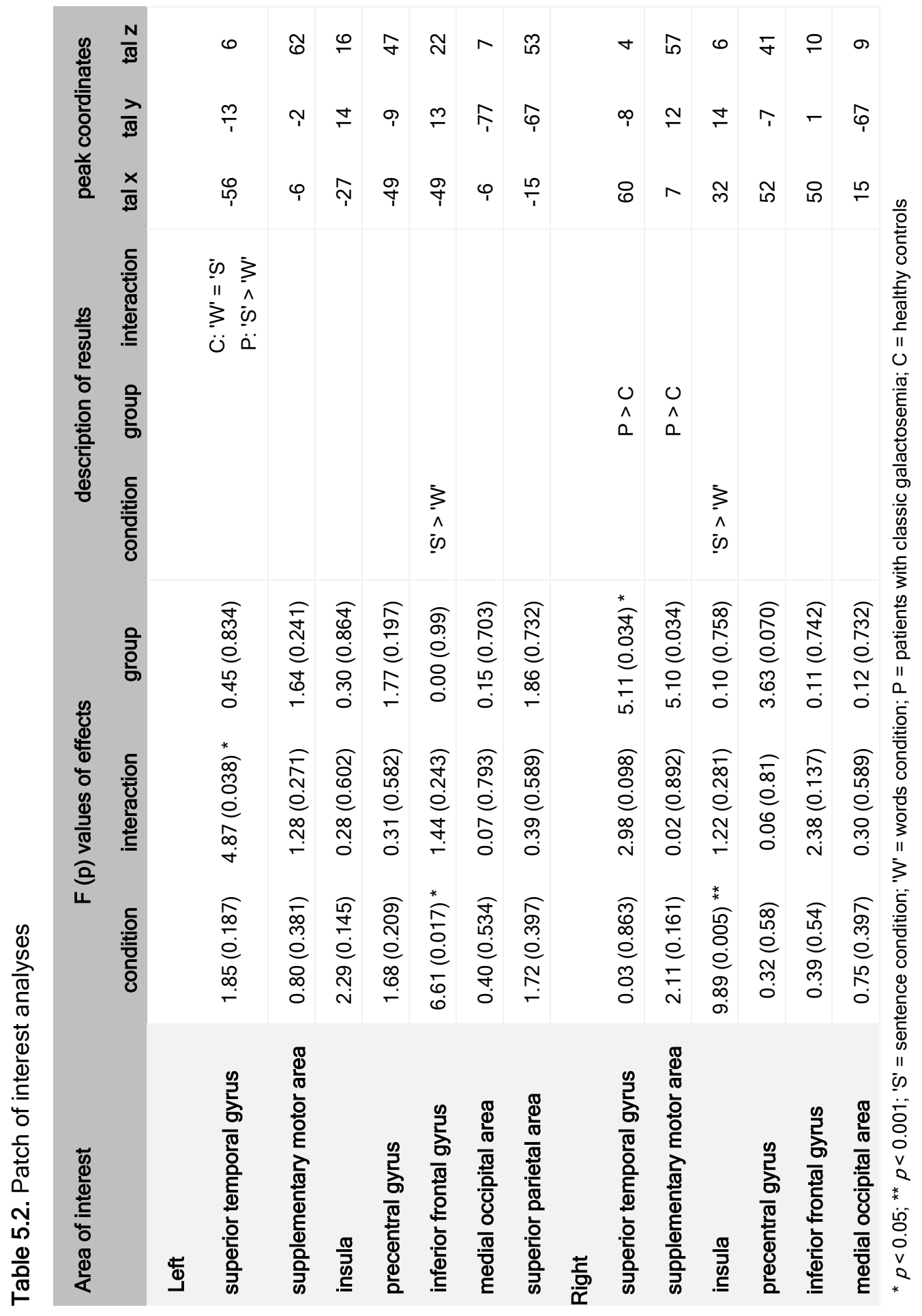




\section{Functional connectivity analysis}

Regions showing a modulation with syntactic complexity - left IFG and right insula - were used as seeds in a correlation analysis. In addition, a seed was placed in left pre-SMA as this region was sensitive to syntactic modulations in healthy adults during the execution of the identical task (Timmers et al., forthcoming). Resulting functional connectivity maps for patients and matched controls are shown in Figure 5.5. Confirming results found in the healthy adults, we observed relatively separate networks correlating with the left IFG and left pre-SMA, respectively.

In the controls, activity in the left IFG seed region correlated with other regions in the left inferior and middle frontal gyrus (MFG), left insula, left PT I supramarginal gyrus (SMG), left posterior superior and middle temporal gyrus (MTG) and right IFG. In the patients, generally a similar network appeared. However, additional regions showed a correlation with the left IFG, including the precentral gyrus and sulcus, more anterior parts of left STG and left MTG, and a larger region in right anterior IFG. The statistical comparison corroborated group differences: stronger connectivity in the patient group was observed with bilateral superior temporal sulcus (STS) / MTG, and with left posterior insula. Weaker connectivity was observed with right PT, and bilateral superior parietal regions.

The activity of the right anterior insula seed correlated with activity in right IFG and MFG, left anterior insula, and bilateral anterior cingulate cortex (ACC) in controls. In patients, the same correlation network was observed, but a more extensive network appeared, involving the right precentral and postcentral areas, and right STG. Statistical comparisons revealed stronger connectivity in the patient group with left PG and posterior MFG; and group differences in bilateral parietal areas. In addition, weaker connectivity was found with bilateral central sulcus.

Finally, the connectivity maps showed that the left pre-SMA seed was functionally connected with bilateral (pre)SMA, bilateral PG, bilateral STG, left $\mathrm{PT}$, and left superior parietal regions. In patients, this finding was again similar. However, in contrast to the controls, the group maps showed that in patients the seed region correlated more extensively with bilateral (pre)SMA, and PG, and less with bilateral STG and left PT. Statistical tests revealed indeed stronger connectivity in the patient group with bilateral (pre)SMA, left MFG, right PT and insula, and weaker connectivity with left posterior STG and MTG, and with bilateral parieto-occipital regions. 

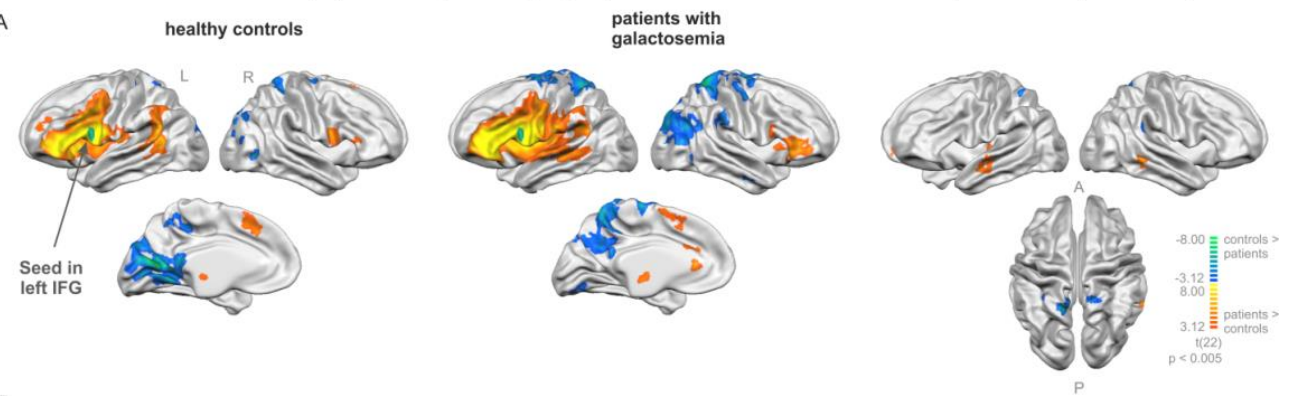

B
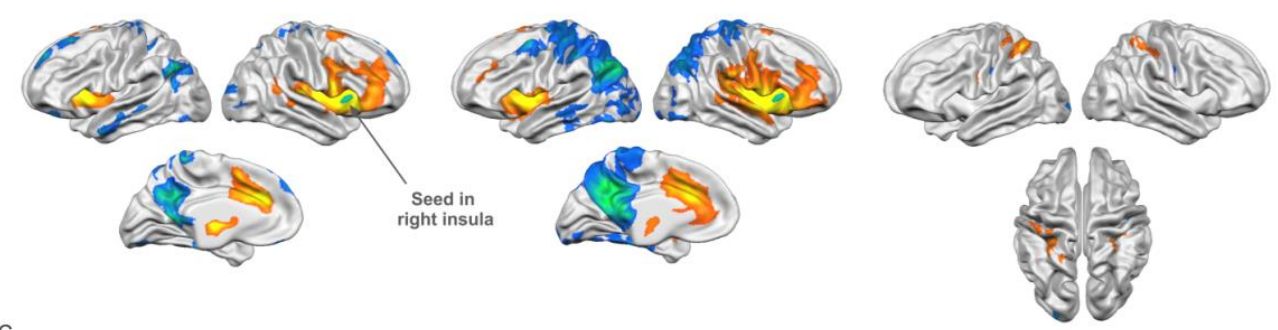

C
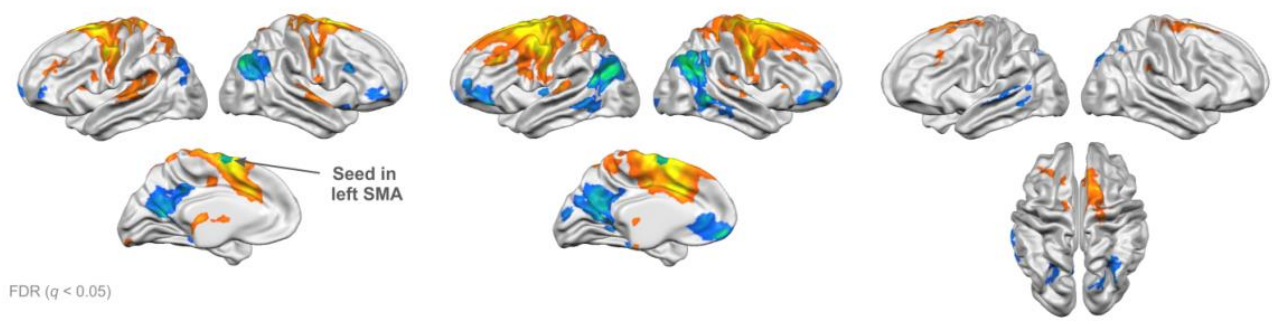

$\operatorname{FDR}(q<0.05$

Figure 5.5. Functional connectivity maps. Left and middle column: Statistical maps resulting from the seed-based functional connectivity analysis, separate per group (red indicates positive correlation, blue indicates negative correlation). Seed regions were defined based on observed syntactic complexity modulation: the left IFG, right insula (see Table 5.2). In addition, the left (pre)SMA was included as a seed region, as it has been reported sensitive to syntactic modulations in this task in healthy adults (Timmers et al., forthcoming). The seeds are overlaid in green. Maps are thresholded at FDR q < 0.05. Right column: Group differences in seed-based connectivity maps across the cortical surface, per seed region. In red, areas are shown in which patients showed stronger connectivity compared to controls; in blue regions, patients showed weaker connectivity compared to controls. Maps are thresholded at $p<0.005$.

\section{Discussion}

The current study investigated functional networks associated with language production deficits in patients with classic galactosemia, a disease of galactose metabolism. For the first time in this patient group, a sentence production paradigm was combined with functional MRI. This allowed us to study brain 
areas and neural networks involved in processing this task. One main finding was that the patients recruited a generally similar network during language production, as compared to controls. This finding is in line with our previous EEG study, in which we used event-related potentials (ERPs, derivatives from the EEG) to study the time course of syntactic encoding with the identical paradigm. An ERP is an electrophysiological measure of the brain's response time locked to a certain stimulus or task. The ERP data of patients and controls showed a similar morphology of the ERP waveform, indicating similar neural sources of brain activity over time between the two groups (Timmers et al., 2012).

A second main finding was that within this target network associated with language processing, we observed specific differences in neural activity and functional connectivity patterns between patients and controls. This is again in line with ERP findings that showed alterations between groups and conditions in specific time windows sensitive for syntactic encoding, but also in time windows related to conceptual and lexical planning. The current fMRI data showed that patients - in comparison to matched controls - involved more extensive, and additional areas (in left frontal cortex, PG and SMA; and right insula, respectively), while involving other areas less (left STG, PT). A similar pattern was observed in functional connectivity maps of the patients, showing stronger connectivity with regions in frontal and motor cortex, and weaker connectivity with posterior superior temporal regions, compared to controls. Together, the data present the first evidence of specific affected neural activity and connectivity during language production in this patient population.

The data revealed that both groups showed a modulation with syntactic complexity in left IFG (higher activity for sentences 'S' compared to words 'W'). This finding supports previous studies with healthy controls (e.g., Indefrey et al., 2001; Segaert et al., 2011, Timmers et al., forthcoming). Together with the absence of a group effect in activity level in left IFG, it indicates that the left IFG is similarly associated with syntactic planning in both groups. The functional connectivity patterns with the left IFG seed region, however, showed differences across the groups. The patients showed stronger connectivity with bilateral STS/MTG, compared to controls. Comprehension studies have found functional interactions between left IFG and left posterior MTG (Snijders et al., 2009). The authors suggested that the left IFG subserves online integration of lexicalsyntactic information into sentence structure, while activity in posterior MTG reflects retrieval processes of lexical-syntactic information from the mental lexicon. Stronger connectivity between the left IFG and the posterior MTG in patients with galactosemia could reflect increased effort during lexical-syntactic 
processes, probably related to verbal working memory load. This would be in line with previous EEG findings of increased ERP amplitudes during time windows associated with lexical planning (Timmers et al., 2012).

In the univariate language contrast (language production versus baseline), we observed less signal change in the PT. This points towards aberrant sensorymotor integration, a function associated with both PT (see Hickok and Poeppel, 2007; Hickok, 2009). This finding would be in line with previously reported motor speech disorders in galactosemia (Potter, 2011; Shriberg et al., 2011). Hypoactivity of the posterior STG and PT has been observed in patients with a specific language impairment (SLI) (Badcock et al., 2012), and with childhood apraxia of speech (CAS) as well (Liegeois et al., 2003). This idea finds support by our findings of a weaker connectivity in patients between IFG as seed region and the right PT, and bilateral superior parietal regions. Hence both the fMRI and the functional connectivity pattern suggest a suboptimal communication between frontal areas associated with overall language planning (IFG) and temporal and parietal sensory-motor integration (PT, superior parietal region).

Another effect of syntactic modulation was found in the right insula, a region mainly recruited in patients. Activation of the right insula during syntactic planning has been found in few earlier studies as well (Haller et al., 2005; Sahin et al., 2006), although it has been suggested that insula activity is associated more with articulation aspects instead of pure syntactic planning (Sahin et al., 2006). A recent review indicates that the left anterior insula is associated mainly with planning of articulation, rather than execution (see Price, 2010; 2012), and in specific with generalized orofacial functions (e.g., lip and tongue movement, and vocalization; Brown et al., 2009). Damage to this region can result in apraxia of speech (Ogar et al., 2005). In addition, the (middle posterior) insula was found to functionally connect to motor and sensory-motor regions, suggesting involvement in sensory-motor integration (Cauda et al., 2011). The current data revealed that patients showed stronger connectivity between the right insula and regions in the left PG and posterior MFG, and bilateral parietal areas, as compared to controls. These findings again suggest alterations in neural activity patterns related to motor speech planning. Open to this point is whether this insula network has evolved in patients over time to compensate for lack of connectivity between IFG and PT/parietal areas.

Furthermore, patients showed an additional syntactic modulation effect in left STG, while the controls did not. This is in contrast with the rather consistent finding of less STG recruitment in this task. To recapitulate, patients show higher signal change in right STG during language production, lower signal change in the left STG, but show a modulation with syntactic complexity in left 
STG, that is absent in the matched controls. The left STG, as far as we know, has not been reported in production studies modulating syntactic complexity. It has been reported in language comprehension studies as relevant for understanding syntactically complex sentences, and for the integration of semantic and syntactic information (see Grodzinsky and Friederici, 2006, for an overview). Recruiting and involving additional areas for syntactic planning indicates the need for additional neural recourses. As language comprehension in the patients is rather intact, it may well be that STG is recruited in patients for production purpose too. An alternative explanation is that the observed modulated reflects increased monitoring in the complex syntactic (sentence) condition as compared to the more simple (words) condition, as speech monitoring is associated with the activity in the STG (Indefrey and Levelt, 2004; Christoffels et al., 2007; 2011).

In contrast to healthy adults performing the same task (Timmers et al., forthcoming), the patients and the matched adolescent controls did not show a syntactic modulation in left pre-SMA. Other studies have found syntactic effects in the pre-SMA as well, and have interpreted these effects in terms of sequencing processes (Segaert et al., 2011). Thus, activity in pre-SMA might be more motor-related than purely language-related. Consistent with that suggestion, the seed in left pre-SMA was functionally connected with bilateral SMA, PG, STG, and left PT and superior parietal regions. In the patients, using pre-SMA as seed region, we observed stronger connectivity with bilateral SMA, MFG, right PT and insula, compared to controls. As these regions are all implicated in the (sensory)motor system, this finding further supports the notion of altered motor planning during speech production in these patients.

The overall pattern of the results suggests abnormalities associated with syntactic planning (in the IFG/MTG/STS network), as well as with (sensory-) motor planning of speech production (SMA/insula/STG/PT network). The latter finding is not entirely unexpected, given previous studies reporting motor speech impairments, such as childhood apraxia of speech, dysathria, and respiratory/phonatory difficulties (Potter, 2011; Shriberg et al., 2011), movements impairments (e.g., tremor, dystonia) (Rubio-Agusti et al., 2013), and co-occurring motor and speech disorders (e.g., speech, coordination and strength disorders) (Potter et al., 2013). In addition, 92\% of the patients in the current study needed speech therapy, and $42 \%$ needed physical therapy at one point in life. The findings further indicate that the employed paradigm was able to capture both the cognitive and the motor aspects of language production, reflected in the relatively separated networks functionally connected to the left IFG, and right insula/left SMA, respectively. 
The higher activations, stronger connectivity as well as the more extensive network for language production - and specifically syntactic production - might have several reasons. First, it might reflect adaptation mechanisms of the patients to cope with cognitive difficulties in the task (our previous study demonstrated lower performance of the patients on this particular task, Timmers et al., 2012). Higher cognitive demands might lead to a higher signal change within an area, but it might also result in consulting additional cognitive resources related to attention and working memory (see Harvey et al., 2005, for an example analogy in major depressive disorder). Alternatively, the finding might reflect general neural compensation. For example, we observed that the right hemisphere was more involved in the patient group as compared to the controls. This finding would then be in line with the idea that the right hemisphere is involved in neural compensation for left hemispheric deficits (Moore, 1989).

Moreover, in the right IFG a significant baseline CBF difference was found, and trends towards a difference in all other covered regions (the slab covered bilateral inferior frontal, insular, and superior temporal regions). The CBF was higher in the patient group compared to the controls. A higher baseline level of CBF results in lower sensitivity of the BOLD signal, hence a smaller BOLD signal change for the same amount of neuronal activity change (Brown et al., 2003). Thus, the observed increases in the BOLD signal in the patients are not explainable by the observed baseline CBF differences.

An explanation for the observed CBF findings (higher in patients compared to controls) is still lacking. In a PET study in patients with galactosemia, the authors also observed increases in baseline glucose metabolism in the cingulate and temporal regions (Dubroff et al., 2008). This would be in line with a tight linkage between baseline CBF and baseline glucose metabolism. However, more prominent in the PET study were widespread decreases in glucose metabolism, including in the STG, medial occipital lobe and superior frontal cortex, which contrast the current findings of increased CBF. However, as the PET study differed in many respects (spatial resolution, age of the patients, sample size), it is difficult to draw direct comparisons.

In terms of pathophysiological mechanisms, researchers have suggested involvement of myelin deficits (Nelson et al., 1992), as well as altered signalling due to myo-inositol abnormalities (Berry, 2011). Myelin abnormalities have been found to be relatively widespread across the brain (Nelson et al., 1992), although a recent diffusion weighted imaging study from our group observed specific regional differences (Timmers et al., submitted). As a result of affected myelin, neural information transfer will be less efficient, hence might result in 
more spread activity patterns. It cannot explain, however, the recruitment of additional regions in both language production in general and syntactic planning. In addition, other mechanisms are required to explain why some brain areas are more affected than others. It has been suggested that epigenetic factors are involved (Coman et al., 2010), and future studies have to be conducted in order to link these expression profiles to the cognitive profile observed in galactosemia. Variations in genetic profile have already been linked to relatively specific language impairments (e.g., mutations in FOXP2 gene, see Liegeois et al., 2003).

To conclude, for the first time we show altered neural activity and connectivity during active language performance in classic galactosemia. Both the patient and control group showed syntactic planning modulations in left IFG, but the patients recruited additional areas for this function. Furthermore, we observed several specific group differences in the neural activation and functional connectivity patterns. We showed that these differences are not related to baseline differences between groups in CBF. In addition to aspects of higher cognitive load and working memory, the observed difference could point to compensation mechanisms for disease-based functional alterations within the language network. Full compensation, however, seems to fail in the context of language production. Overall, the results demonstrate that these language production abnormalities in classic galactosemia are associated with both the cognitive counterpart of language production (including syntactic encoding), as well as the motor speech (planning) part. This conclusion is based on the observed alternations in distinct networks for syntactic planning and motor planning in these patients.

\section{Acknowledgements}

We kindly acknowledge the Dutch Galactosemia Patient Association (GVN) who facilitated and supported this study financially (Galactosemia Research Fund, GOF), and cooperated and assisted during the preparation of this study. We further thank the participants and their parents for their time, effort and cooperation. The study was furthermore funded by a Maastricht University incentive (Mobilizing Minds: "Females into higher positions" to BM Jansma). 

<smiles>CCCCCCC</smiles> 
CHAPTER 6

\section{ASSESSING WHITE MATTER MICROSTRUCTURE IN CLASSIC GALACTOSEMIA USING NEURITE ORIENTATION DISPERSION AND DENSITY IMAGING}

Inge Timmers, Hui Zhang, Matteo Bastiani, Bernadette $M$ Jansma, Alard Roebroeck, and M Estela Rubio-Gozalbo (submitted). Assessing white matter microstructure in classic galactosemia using neurite orientation dispersion and density imaging 


\section{Summary}

White matter abnormalities have been observed in patients with classic galactosemia, an inborn error of galactose metabolism. However, the MRI data that has been collected in the past has been generally qualitative in nature. Hence, it has been difficult to obtain a comprehensive overview of the abnormalities, and to find correlations with outcome and behaviour. The current study used high angular, multi-shell diffusion weighted imaging to investigate the white matter microstructure in this disease in more detail. In addition to standard diffusion tensor imaging (DTI) analyses, we applied neurite orientation dispersion and density imaging (NODDI). This analysis technique was designed to estimate neurite density and orientation dispersion, two key contributors to the widely used fractional anisotropy (FA, derived from DTI), that are furthermore biologically interpretable. Results showed extensive white matter abnormalities: neurite density index (NDI) was lower in the patient group in bilateral anterior areas, and orientation dispersion index (ODI) was increased mainly in the left hemisphere. The specific regional profiles are in general agreement with the cognitive profile observed in galactosemia showing higher order cognitive impairments, and language and motor impairments, respectively. Moreover, the white matter properties correlated with disease variables (i.e., age, age at onset of diet) and with behavioural outcome (e.g., visual working memory). To conclude, this study provides the first quantitative evidence of white matter pathology with respect to both density and dispersion of neurites in these patients. The results are discussed in light of suggested pathophysiological mechanisms. 


\section{Introduction}

White matter (WM) pathology has been repeatedly observed in classic galactosemia, an inherited disorder of galactose metabolism due to severe galactose-1-phosphate uridylyltransferase (GALT) deficiency (Holton et al., 2001). Neonates develop acute symptoms following the ingestion of galactose. A galactose-restricted diet resolves the acute symptoms, but long term complications still occur in these patients, including language production impairments, speech (motor) abnormalities, slower information processing, memory and executive functioning deficits, and generally a lower intelligence level, despite compliance with the dietary galactose restriction (Antshel et al., 2004; Doyle et al., 2010; Potter et al., 2008; Potter, 2011; Rubio-Agusti et al., 2013; Timmers et al., 2011; Waisbren et al., 2012).

The first extensive study on magnetic resonance imaging (MRI) appearance revealed signal hyperintensities on T2-weighted (anatomical) images in the majority of patient's peripheral cerebral and cerebellar WM (Nelson et al., 1992), with normal signal in internal capsule and corpus callosum. Widespread small lesions were found in the WM (17\%), with a tendency to be clustered around the lateral ventricle. Further, mildly enlarged lateral ventricles (33\%), and signs of cerebellar atrophy (13\%) were observed. The authors postulated that the abnormal signal intensity was due to a primary abnormality in the biochemical structure of myelin secondary to abnormal and/or deficient galactocerebrosides. Histopathological and biochemical examination in an untreated adult patient with galactosemia revealed low galactocerebroside levels, which support this idea (Haberland et al., 1971). Later studies continued to observe white matter abnormalities (Crome, 1962; Hughes et al., 2009; Otaduy et al., 2006; RubioAgusti et al., 2013; Wang et al., 2001), and links with affected myelination (Böhles et al., 1986; Widhalm et al., 2002).

Until now, collected MRI data of patients with galactosemia has been generally qualitative in nature. The lack of quantification makes it difficult to obtain an accurate picture of the abnormalities, to find correlations with outcome and to compare across studies. To investigate WM pathology in more detail, and in a quantitative manner, diffusion-weighted imaging (DWI) can be used to assess properties and potential abnormalities in tissue microstructure. By modelling the diffusion of water molecules, many different parameters can be estimated. Most widely known is fractional anisotropy (FA), based on diffusion tensor imaging (DTI). FA concerns the degree of anisotropic diffusion, which is higher in WM (because of coherently formed fibre bundles) compared to grey matter (GM). In numerous diseases, reductions in FA have been found, and linked to axonal degeneration (e.g., in amyotrophic lateral sclerosis, Chapman et al., 2013) or 
myelin breakdown (e.g., in multiple sclerosis, Roosendaal et al., 2009). FA has been shown to be a very sensitive measure, but is inherently non-specific (Pierpaoli et al., 1996) as a reduction could be caused by a reduction in neurite density, an increase in dispersion of orientation, and several other factors. To disentangle these key contributors to FA, an approach called neurite orientation dispersion and density imaging (NODDI) was developed (Zhang et al., 2012). By distinguishing three compartments (intra-, extra-neurite, and cerebral spinal fluid) that are each modelled in a biologically informed manner, these parameters can be estimated and analysed individually. Measures of neurite density and orientation dispersion in WM and GM have shown great correspondence to histological measures such as optical myelin staining intensity (Jespersen et al., 2010), and quantitative Golgi analysis (Jespersen et al., 2012), respectively. In WM, orientation dispersion quantifies the bending and fanning of axons and changes in neurite morphology have been implicated in diseases, although still mainly studied histologically in post-mortem tissue. In multiple sclerosis, for instance, axonal loss, reflected by reductions in axonal density and axonal area has been found in normal appearing WM (Evanglou et al., 2000). The correlation between FA and neurite density, however, is relatively weak, suggesting that for diseases primarily affecting axonal density, neurite density might be a more sensitive marker of pathology compared to FA. Successful and reliable estimation of neurite density and orientation dispersion has been shown in previous studies (Assaf and Basser, 2005; Zhang et al., 2012), and a recent clinical study has demonstrated its usefulness in localisation of cortical malformations in epilepsy patients (Winston et al., 2013). In the current study, we applied NODDI to a patient cohort with classic galactosemia to study white matter microstructure and establish relationships with the observed cognitive profile. Because of the limited available literature, we took an explorative approach and focused on potential changes in the main white matter tracts of the brain.

\section{Methods}

\section{Participants}

Eight patients with galactosemia and eight healthy controls participated in this study. Classic galactosemia was diagnosed by GALT enzyme activity assay and/or GALT-gene mutational analysis, and all patients adhered to a galactose restricted diet (see Table 6.1). Characteristics of the groups can be found in Table 6.1. Participants had no other relevant health conditions, were screened for MRI compatibility, and signed informed consent (in case of minors, both 
parents/caregivers also gave written informed consent). The Medical Ethical Committee of the Maastricht University Hospital/Maastricht University gave ethical clearance for this study.

Table 6.1. Participant characteristics

\begin{tabular}{|c|c|c|c|c|}
\hline & \multicolumn{2}{|c|}{ patients } & \multicolumn{2}{|c|}{ controls } \\
\hline & Number & range & number & range \\
\hline group size & 8 & & 8 & \\
\hline males / females & $2 / 6$ & & $3 / 6$ & \\
\hline age (in years) ${ }^{1}$ & 17.9 & $15.9-21.2$ & 17.2 & $14.7-20.0$ \\
\hline $\begin{array}{r}\text { GALT activity } \\
\text { (in } \% \text { of reference value) }^{2}\end{array}$ & $0.54 \%$ & $0-1.52 \%$ & & \\
\hline GALT mutation & $\begin{array}{c}4(50 \%) \\
2(25 \%) \\
1(12.5 \%)\end{array}$ & $\begin{array}{l}\text { Q188R/Q188R } \\
\text { 400Tdel/unknown } \\
\text { L195P/K229N }\end{array}$ & & \\
\hline $\begin{array}{r}\text { urine galactose } \\
\text { concentration } \\
\text { (in } \mu \mathrm{mol} / \mathrm{mmol} \text { creatinine) }\end{array}$ & 11.9 & $N D^{3}-33$ & & \\
\hline $\begin{array}{r}\text { urine galactitol } \\
\text { concentration } \\
\text { (in } \mu \mathrm{mol} / \mathrm{mmol} \text { creatinine) }\end{array}$ & 140 & $97-187$ & & \\
\hline $\begin{array}{l}\text { age at initiation of diet } \\
\text { (in days) }\end{array}$ & 11.8 & $0-35$ & & \\
\hline $\begin{array}{r}\text { visual working memory } \\
\text { (t-score) }{ }^{4}\end{array}$ & 32.3 & $22-51$ & & \\
\hline $\begin{array}{r}\text { sustained attention } \\
(\text { mean RT) })^{5}\end{array}$ & $13.8 \mathrm{~s}$ & $11.3-18.1 \mathrm{~s}$ & & \\
\hline $\begin{array}{r}\text { verbal working memory } \\
\text { (norm score) }{ }^{6}\end{array}$ & 3.9 & $3-7$ & & \\
\hline $\begin{array}{l}\text { voice onset time sentence } \\
\text { production (in seconds) }{ }^{7}\end{array}$ & $1.97 \mathrm{~s}$ & $1.49-2.20 \mathrm{~s}$ & & \\
\hline
\end{tabular}

\footnotetext{
${ }^{1}$ Age did not differ significantly between the groups $\left[F_{1,16}=0.44, p=0.519\right] .{ }^{2}$ GALT activity was measured at diagnosis; ${ }^{3} \mathrm{ND}=$ not detectable; ${ }^{4}$ as assessed by Rey Osterreith Complex Figure Immediate recall (Meyers and Meyers, 1995); ${ }^{5}$ as assessed by mean reaction time in Bourdon-Vos task (Vos, 1988); ${ }^{6}$ as assessed by Digit Span subtest of WISC-R [norm score has mean of 10, SD
} 
of 3] (van Haasen et al., 1986); ${ }^{7}$ average voice onset time in a sentence production task (see Timmers et al., 2012, for more information on these behavioural measures)

\section{Procedures}

The session started with acquainting the participants in a mock (dummy) scanner (Maastricht University), where participants received information on the scanning procedures and safety measures. After safety precautions were taken, the participant was placed comfortably inside the real scanner. Through a mirror attached to the head coil, participants were able to see a screen on which an entertaining movie was projected during the acquisition (to distract and minimize movements). The DWI was part of a larger project (other data will be described elsewhere).

\section{Data acquisition}

Data were acquired on a 3-T Siemens Trio whole body scanner (Siemens Medical System, Erlangen, Germany), using a 32-channel head coil. The DWI data were obtained using a doubly refocused single-shot spin echo EPI sequence. 64 slices with a voxel-size of $2.2 \mathrm{~mm}$ were obtained $(T R=8500 \mathrm{~ms}$; $\mathrm{TE}=97 \mathrm{~ms}$ ). Data were acquired at two different $b$-values: $b=1000 \mathrm{~s} / \mathrm{mm}^{2}$ with 64 diffusion-encoding gradient directions and $b=2000 \mathrm{~s} / \mathrm{mm}^{2}$ with 64 diffusion directions. In addition, $5 b=0$ images were collected, two of which were acquired using a reversed phase encoding direction (posterior to anterior), to allow the estimation of susceptibility induced distortions. The diffusion encoding directions spanned the entire sphere.

\section{Data analyses}

Pre-processing of the data started with estimating susceptibility induced distortions. As part of the data was acquired using reversed phase-encode directions, pairs of images were available with distortions going in opposite directions. From these pairs, the susceptibility-induced off-resonance field was estimated using a method similar to the one described in Andersson et al. (2003) (topup of FMRIB Software Library [FSL], Smith et al., 2004). Further, eddy current-induced distortions and subject motion were estimated, and all distortions were corrected, by simultaneously modelling the effects of diffusion eddy currents (using a Gaussian process) and movements on the image (using FSL's eddy). Using each participant's output from the eddy method, the bvectors were rotated to account for the corrections. 
From one shell of the corrected DWI data (b1000), the diffusion tensors were estimated using a linear fitting algorithm (dtifit, implemented in FSL). DTI-TK was used for tensor-based spatial normalization of the volumes to an iteratively optimized template (Zhang et al., 2006). The algorithm applies a deformable registration to the tensor images, resulting in improved registration as compared to FA-based registration algorithms (Keihaninejad et al., 2013). The resulting normalized images were averaged, and high-resolution FA maps (1 $\mathrm{mm}$ isovoxel) were derived. The mean FA image was thinned to create a mean FA skeleton, representing the centres of all tracts common to the group (as implemented in tract based spatial statistics [TBSS] of FSL, Smith et al., 2006). Each subject's aligned data was then projected onto this skeleton using calculated distance maps, and the resulting data were fed into the statistical analysis.

In parallel, neurite orientation dispersion and density imaging (NODDI) was applied to the pre-processed data. The NODDI tissue model distinguishes between three compartments: 1) intra-neurite space, representing neurites (space bounded by neurite membranes) and modelled as restricted diffusion (in particular, sticks, incorporating orientation dispersion utilizing a Watson distribution); 2) extra-neurite space, surrounding the neurites (occupied typically by glia cells and somas in GM), which is modelled as hindered, but not restricted diffusion (anisotropic Gaussian diffusion); and 3) cerebral spinal fluid (CSF), modelled as isotropic Gaussian diffusion. The main resulting parameters of NODDI are: neurite density index (NDI), derived from the intra-neurite volume fraction (typically high in WM, low in GM); and orientation dispersion index $(\mathrm{ODI})$, which quantifies the angular variation of neurite orientation (ranging from 0 for perfectly coherently oriented structures, to 1 for isotropic structures; typically high in GM, low in WM). The output scalar images from NODDI were normalized to the - already defined - study-specific common group space using the transformation fields as calculated per participant during the tensor-based registration. Then, the normalized NDI and ODI data were projected onto the already calculated- mean FA skeleton using the original distance maps.

On the skeletonised FA, NDI and ODI maps, permutation-based statistics were carried out (as implemented by randomise in FSL; 5000 permutations). First, a design was used having group as a between-subjects factor, and age as a covariate. Second, correlations with several disease and available behavioural outcomes (see Table 6.1) were examined across the skeleton and within regions of interest. P-values were corrected by means of the Threshold-Free Cluster Enhancement (TFCE) option (Smith and Nichols, 2009). A corrected alpha level of 0.05 was considered as significant. 


\section{Results}

\section{Differences across groups}

On the left columns of both sides in Figure 6.1, the mean FA maps are shown with the mean FA skeleton. Superimposed are the significant group differences, which are observed across the majority of the WM tracts, except for the cerebellar tracts.

In the middle columns of Figure 6.1, the mean NDI maps, the skeleton and corresponding group differences are shown. Comparing these differences with FA, one can notice that the density changes overlap with FA changes, but are more localized. NDI changes were found mainly bilateral and located mostly in the anterior part of the brain. In Figure 6.2, the NDI group differences are overlaid on colour maps in coronal and sagittal orientations, aiding in the localization of the tracts (using Wakana et al., 2003). Affected tracts include the anterior part of the corpus callosum (CC) and forceps minor (bilateral), corona radiata (bilateral), part of the internal (IC; right) and external capsula (EC; right), uncinate fasciculus (UF; bilateral), superior longitudinal fasciculus (SLF; bilateral) and inferior longitudinal fasciculus (ILF; right).

On the right-sided columns in Figure 6.1, group differences in ODI are displayed, overlaid on the mean ODI maps and the skeleton. Again, the ODI changes are overlapping with FA, but more specifically localized. The dispersion changes are mainly located on the left, middle parts of the brain and show minimal overlap with the NDI changes (see Figure 6.2 for an overlay of the results on coronal and sagittal colour-coded images). Affected tracts include the CC, corona radiata (bilateral) and IC (cortico-spinal tract), EC, SLF, and ILF.

In order to examine the cerebellum irrespective of the skeleton, ROls were manually drawn based on the group averaged FA map: two in bilateral middle cerebellar peduncles (one more anterior, one more posterior). No group differences were found in FA, NDI or ODI (all $p>0.3$ ). 


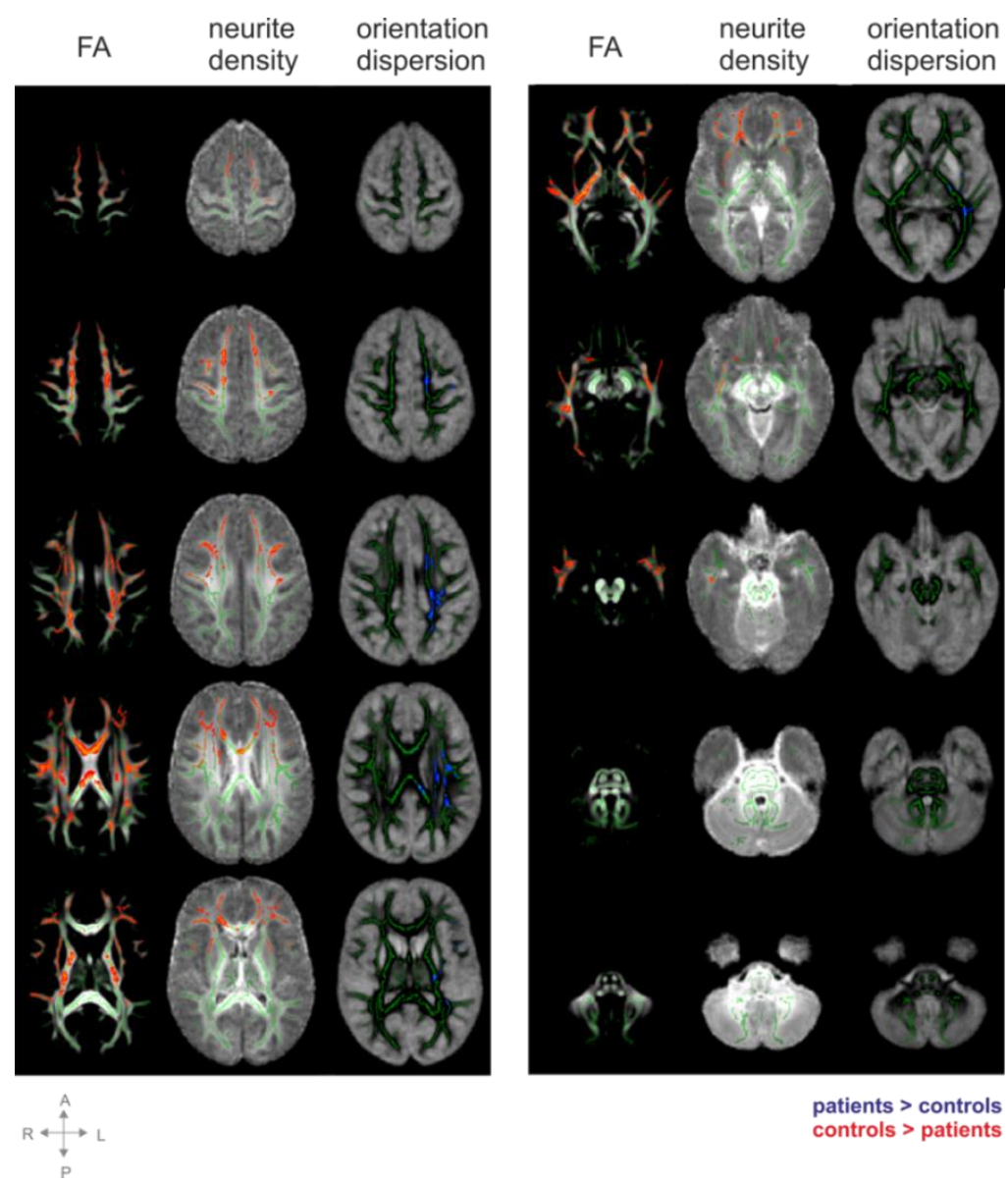

Figure 6.1. Group differences in main white matter tracts. Average group maps of the FA values, neurite density index (NDI) values, and orientation dispersion index (ODI) values (all ranging from 0.2 - 0.8), in transversal slices covering the majority of the brain from superior to inferior. Superimposed are the mean FA skeleton (green) and the statistical group differences (red: controls $>$ patients; blue: patients > controls). Presented results are TFCE-corrected and thresholded at a corrected alpha-level of 0.05 . For display purposes, the results are thickened by filling it out into the local tracts (as implemented in TBSS). Note that left is right in these images.

\section{Correlations with disease and behavioural variables}

FA, NDI and ODI did not correlate with age across the skeleton in the controls. ODI values in the patients, however, tended to be higher in older patients in several regions, mostly on the right hemisphere (Figure 6.3). No correlations with age were found for NDI and FA in the patients, or with any of the other behavioural measures. 


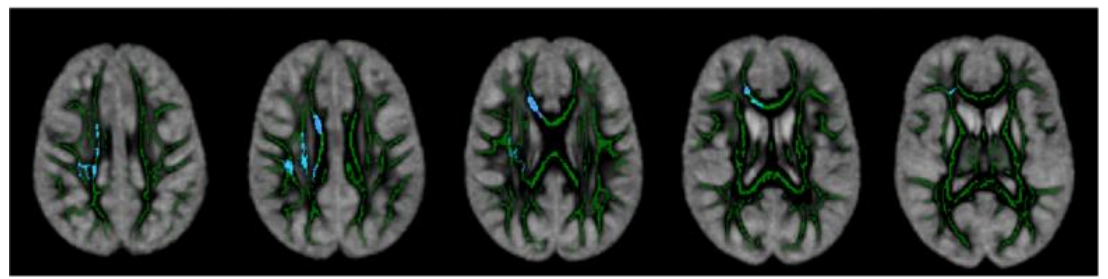

positive correlation

Figure 6.3. Correlations between ODI and age across the skeleton. Significant correlations are overlaid on the mean skeleton and mean ODI map. Depicted in blue are positive correlations (there are no negative correlations). Presented results are TFCE-corrected and thresholded at a corrected alpha-level of 0.05 . For display purposes, the results are thickened. Note that left is right in these images.

Of the areas in which significant group differences in NDI and/or ODI were found, regions of interest (ROIs) were created. Mean parameter values within these regions can be found in Table 6.2. Associations with age (in both groups), and with several other variables were calculated (only available for the patients; see Table 6.2). Results showed that ODI in the CC was positively associated with age of the patients. In controls, FA in the left CC, ILF and SLF was associated with age, showing the opposite (negative) relation to that observed in the patients. In addition, the age at onset of diet showed several negative correlations: in bilateral forceps minor (with NDI, only right forceps minor correlated negatively with $\mathrm{FA}$ ), and in EC (with FA). Several other trends ( $p<$ 0.01 ) were observed as well (see Table 6.2). A selection of the correlations is plotted in Figure 6.4. 

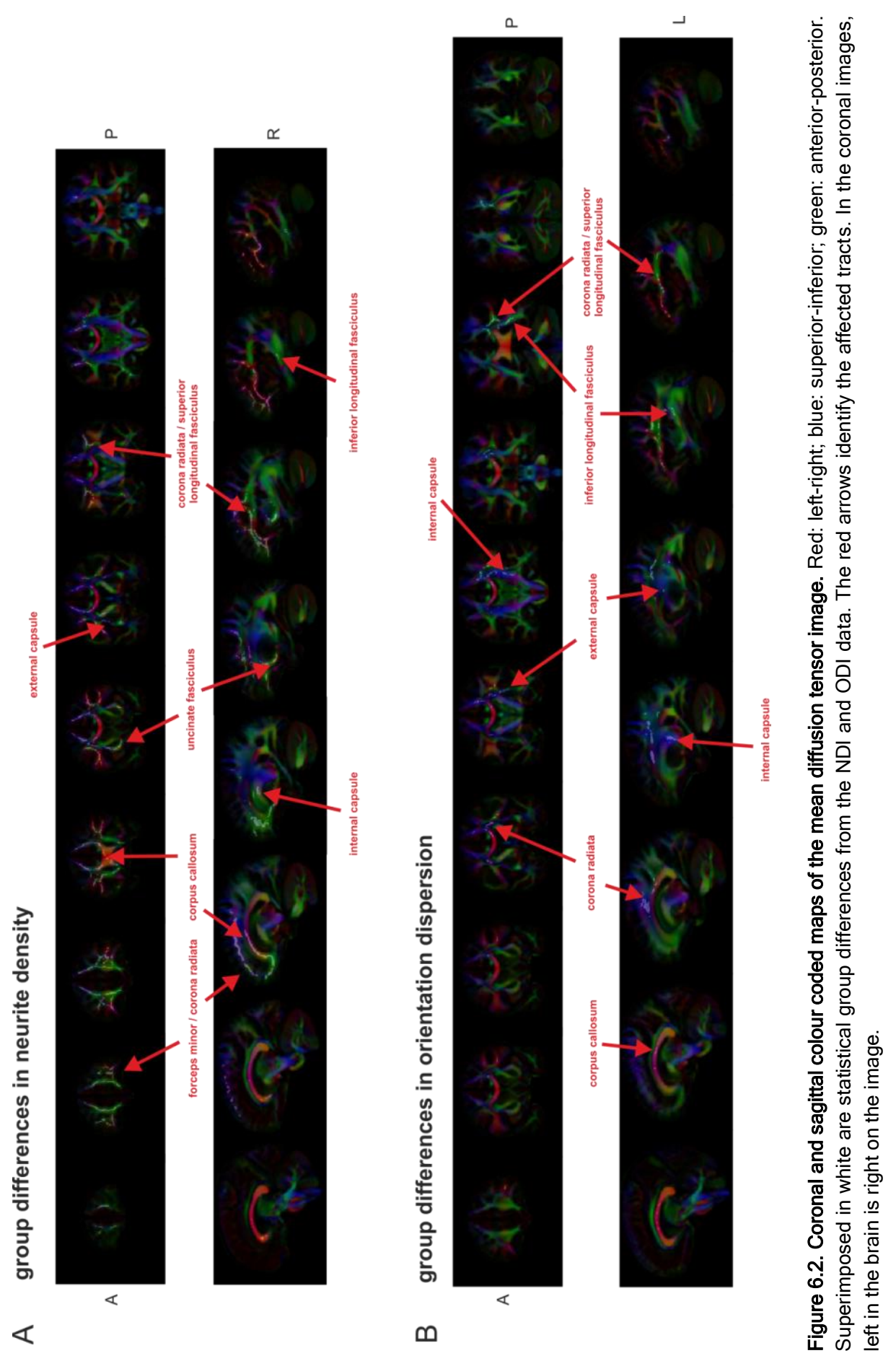


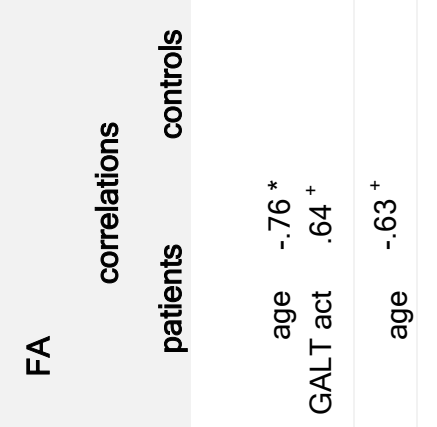

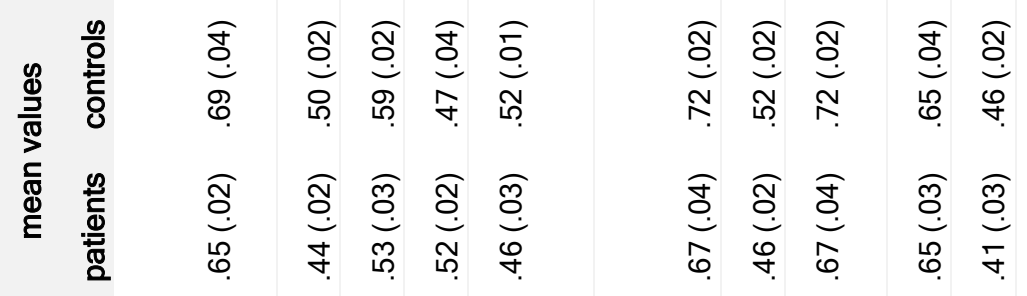

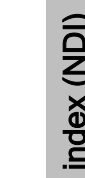

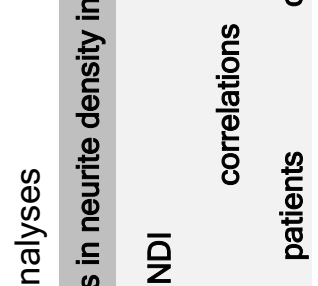

을
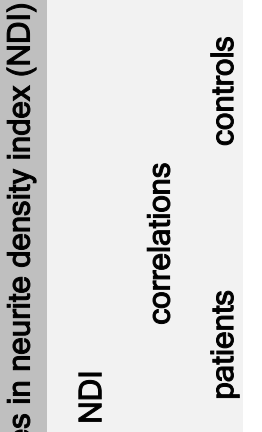

$\stackrel{\infty}{\circ}$

ণ্ঠে

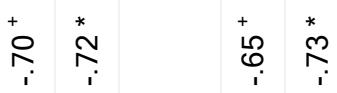

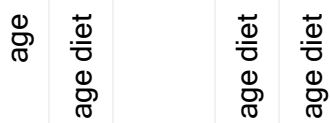

+̊.

ণ্ঠ

$\stackrel{*}{9}$

+ீ

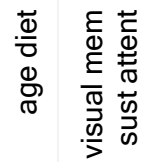

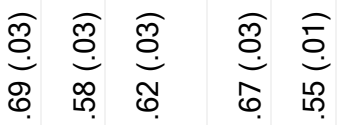

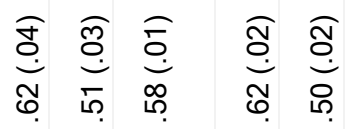

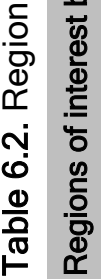

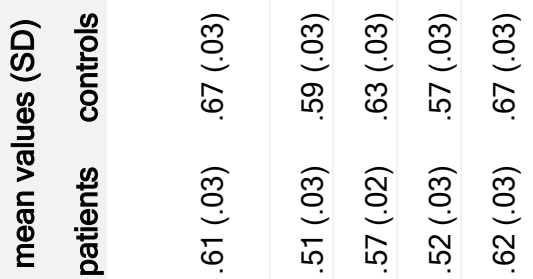

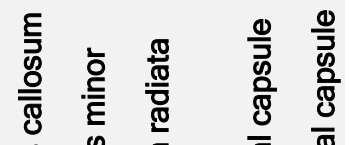

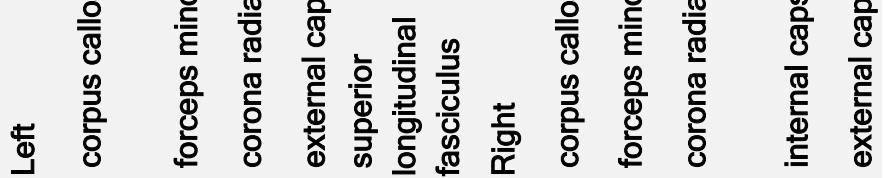

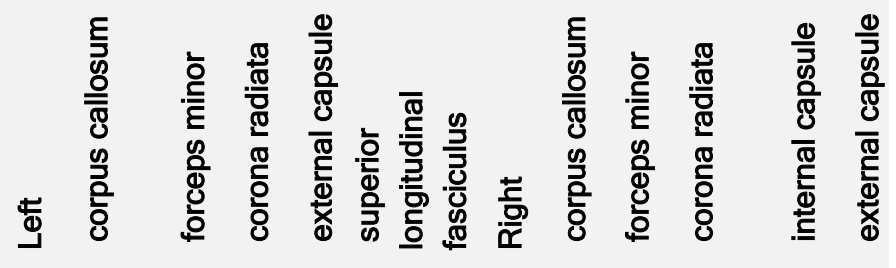




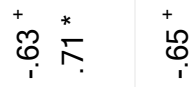

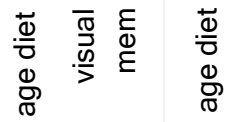

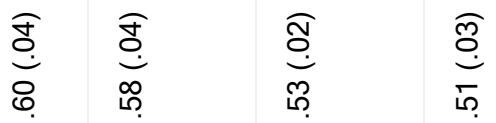

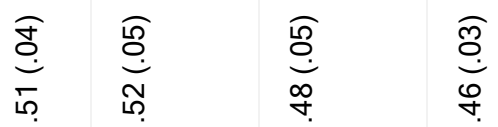

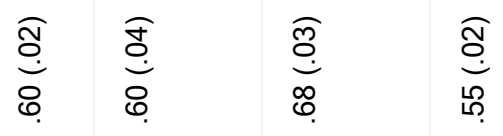

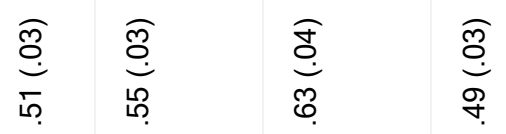

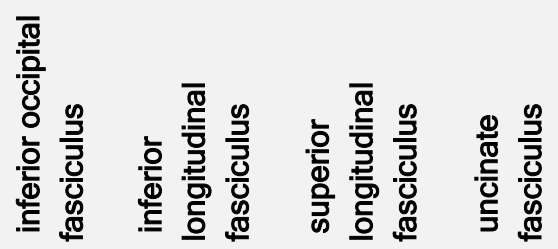



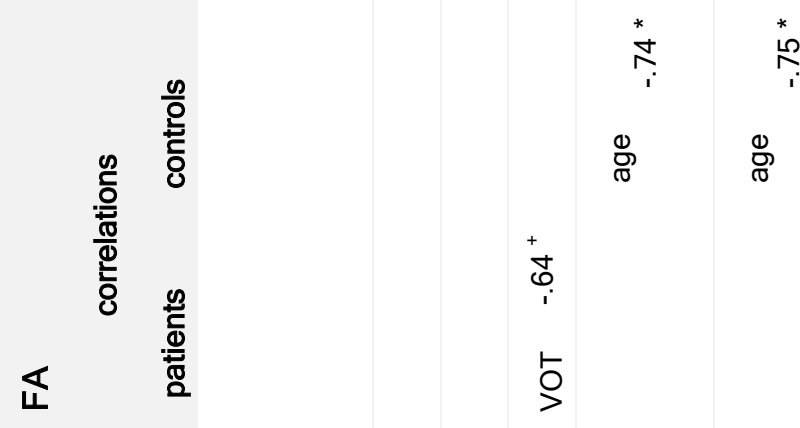

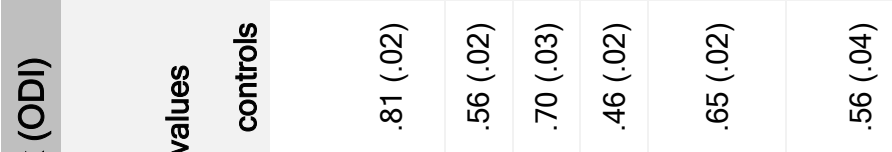

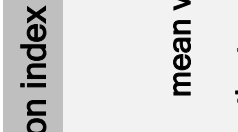

\&

递

은

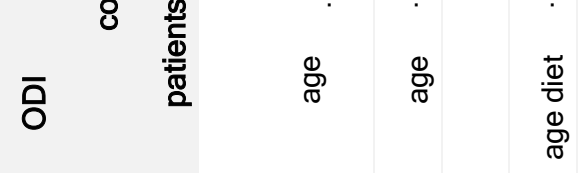

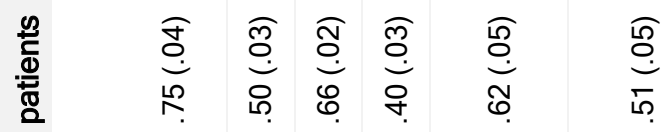

$\stackrel{+}{\infty}$

๑

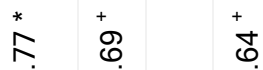

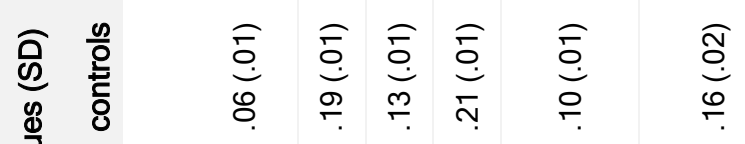

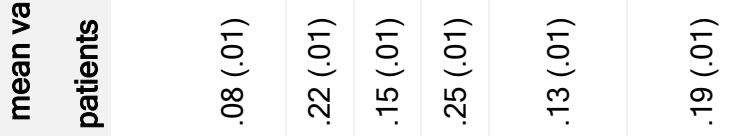

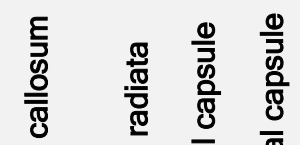

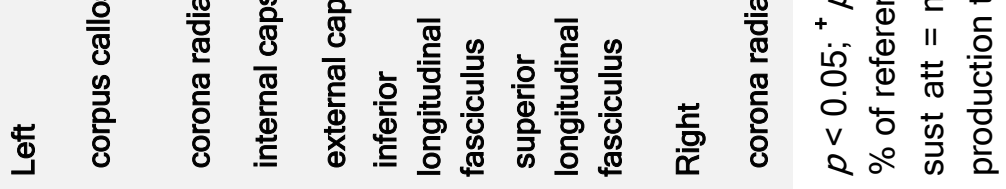


Mean values in the ROls were correlated with available cognitive measures of the patient group as well ${ }^{1}$. Visual working memory performance was positively correlated with FA in right ILF (better performances are associated with higher FA), and several trends were observed and shown in Table 6.2.

A ROls based on NDI group differences
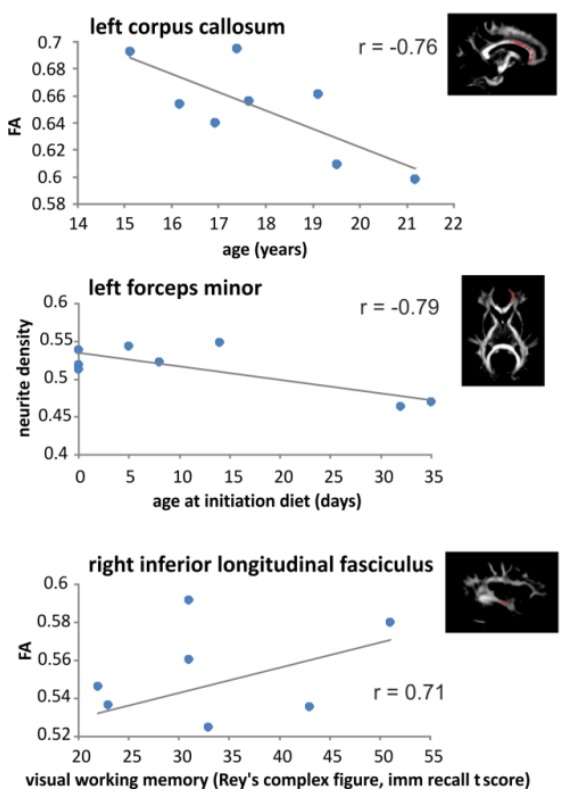

B ROIs based on ODI group differences

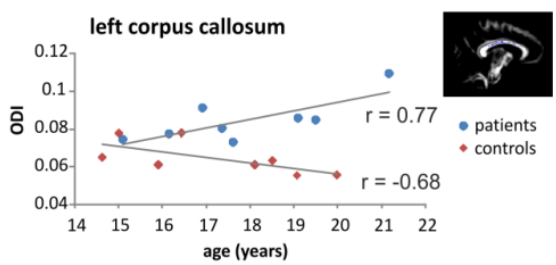

Figure 6.4. ROI correlation analyses. A selection of significant correlations of regions of interests (ROIs) based on group differences in NDI (A) or in ODI (B). Added are a linear trend line, the correlation, and an illustration of the location of the ROI.

\section{Discussion}

The current study has enabled us to investigate for the first time white matter (WM) properties in the main WM tracts of patients with the metabolic disorder classic galactosemia. In addition, the NODDI analysis allowed us to go beyond the standard fractional anisotropy (FA) analyses and include estimations of neurite density and orientation dispersion. Previous studies have consistently found WM abnormalities in these patients, and this study extends these findings

\footnotetext{
${ }^{1}$ All patients are part of a study cohort, and participated in earlier studies as well.
} 
by showing lower FA values, lower neurite density (NDI), and higher orientation dispersion (ODI) in several tracts as compared to the control group. Moreover, we observed correlations of these parameters with disease variables and behavioural outcome.

Extensive differences were found across the patient group and the matched controls. Standard DTI analyses showed lower FA values in the patient group spread over almost the entire cerebrum, illustrating the sensitivity but nonspecificity of FA. Reductions in FA values have been found in numerous studies and have been linked to axonal degradation, myelin abnormalities, or a general lower integrity of the white matter (see Winston, 2012, for an overview). However, lower FA values can be the result of a number of underlying mechanisms. Reduced density, increased orientation dispersion, and other factors can contribute to lower FA values. The NODDI analyses allowed us to disentangle the FA into NDI and ODI, and as expected these measures showed more localized and specific results. Lower NDI was found in multiple tracts, mainly in the anterior parts of the brain (bilateral), and higher ODI was found mostly left-lateralized in multiple tracts. Other studies have found widespread white matter abnormalities in these patients, with a tendency to be clustered around the lateral ventricles (Nelson et al., 1992). Although the current data does not support the notion of small widespread lesions and a patchy nature of the abnormalities, we observed that abnormalities tended to cluster along the lateral ventricles, as for instance the SLF, ILF and IC run alongside this ventricle. In contrast to Nelson et al. (1992), we observed involvement of both the $\mathrm{CC}$ and $\mathrm{IC}$, suggesting that the current method is more sensitive to reveal abnormalities. Surprisingly, we did not observe any abnormalities in the cerebellar WM, not by using the skeleton-approach nor the ROI-approach. However, we cannot exclude that there may be fine-grained differences in cerebellar WM that will be picked up with higher resolution images, larger sample sizes and/or in tract-specific analyses.

The finding that several major tracts are less dense in patients with classic galactosemia could be an indirect result of abnormal myelination. Although diffusion cannot directly assess myelin, a reduction in myelin will result in reduced NDI (i.e., myelin loss increases in the extra-neurite space, which indirectly - leads to a reduction in the - relative - volume fraction of the intraneurite space). In turn, this reduced density could lead to less coherently organized axons, and thus to increased ODI. In other words, increases in ODI could be an indirect result of abnormal myelination as well. In GM, ODI is associated with dendritic branching and hence with density of local synaptic connectivity. In WM, which needs to be coherently oriented to be efficient, 
higher dispersion may be less favourable. Increased ODI in these patients might also be a result of reduced or delayed pruning of the axons. In several regions, however, we observed an increasing ODI with increasing age. Noticeable is that the correlations between $\mathrm{ODI}$ and age were mainly observed in the right hemisphere, while the group differences were largest in the left hemisphere. Speculatively, it might be the case that the ODI values in the left hemisphere showed little variation across participants because of the degradation (floor effect), and hence no proper correlation with age. In the right hemisphere there might have been more variation in ODI values, enabling us to find a correlation with age (see Table 6.2 to compare the standard deviations). Another explanation for the increased orientation dispersion is that it reflects increased branching of axons as a compensation mechanism. Longitudinal designs could give more insight in this matter.

The pattern of the observed abnormalities is generally in line with the described cognitive profile in classic galactosemia. The neuropsychological profile includes motor / speech, language, (working) memory, visuo-spatial, and attention problems (Antshel et al., 2004; Doyle et al., 2010; Waggoner et al., 1990; Waisbren et al., 2012). In general, the left-lateralized nature of the ODI findings is in accordance with the observed language and motor abnormalities, as both are generally left-lateralized as well (Gotts et al., 2013; Kell et al., 2011). More specifically, we observed abnormalities in both the UF (NDI) and the SLF (NDI and ODI). These tracts, or more specifically part of the SFL (also referred to as the arcuate fasciculus, AF), are strongly associated with language processing (Friederici, 2009; Glasser and Rilling, 2008). The AF connects temporal and parietal regions with the frontal lobe. Abnormalities in the AF have been linked to conduction aphasia for instance (Catani and Mesulam, 2008), and underdevelopment of this tract in children goes hand in hand with language processing difficulties (see Friederici, 2009). The finding that longer voice onset times (worse performance) in a language production paradigm tends to be associated with higher orientation dispersion (less favourable) in the SLF is also consistent with the involvement of this tract in the observed language abnormalities. The UF has furthermore been associated with language functions, among other reasons because of its left-lateralized nature (FA values are higher in the left UF), and the regions it connects. It provides a ventral route between inferior frontal regions and more anterior superior temporal regions. Recently, it was suggested that the ventral routes are important for comprehension of language (mapping sounds to meaning) (Saur et al., 2008). In addition, we observed involvement of the IC that contains fibres projecting from the medulla oblongata to the cerebral cortex, and include motor tracts such as 
the corticospinal tracts. We also found abnormalities surrounding the precentral gyrus, the premotor and primary motor area of the brain. These two latter findings are in line with motor abnormalities and motor speech abnormalities that are often reported in patients with galactosemia (Potter, 2011; Potter et al., 2013; Rubio-Agusti et al., 2013; Shriberg et al., 2011).

Furthermore, the anterior nature of the NDI changes and the extensive involvement of the corona radiata fit with a profile showing impairments in higher order processes. For instance, working memory functions are subserved by regions in the prefrontal and inferior frontal cortex, among other regions (Cabeza et al., 2002; Martin and Chao, 2001). Additionally, maturation of white matter in several regions of the frontal lobe correlates with performance in working memory tasks (see Klingberg, 2006), and networks of attention have been shown to involve the SLF and anterior corona radiata (Ge et al., 2013). In the current study, lower performance in behavioural tasks such as visual working memory and sustained attention tended to be associated with lower (less favourable) NDI and FA in the anterior corona radiata.

Several studies in galactosemia have suggested that the cognitive complications progress with age, while others have refuted this claim (e.g., Doyle et al., 2010; Waisbren et al., 2012). In the current study, we found a clear relation with age. When inspecting the main WM tracts (i.e., the entire skeleton), we found several regions showing a negative relation between age and ODI, meaning that ODI tends to be higher in older patients (but not in controls). Also when inspecting the specific regions in which group differences were found, multiple relations with age are observed, showing that lower FA or higher orientation dispersion in mainly the $\mathrm{CC}$, forceps minor and corona radiata tends to be observed in older patients. No or opposite correlations were found in the controls group. Although we are mindful that our sample size was relatively small and the data are cross-sectional in nature, the results seem to indicate a progressive nature, at least with respect to the ODI changes. As earlier reported, we observed a negative relation with the age at onset of the diet. The present data suggests that the older the patient at the onset of the diet, the less favourable the white matter properties are in many WM tracts, including bilateral forceps minor, IC, EC, SLF, ILF, and right corona radiata.

It remains unclear why certain regions are more affected than others. NDI is lower particularly in anterior regions. One potential explanation is that tracts that mature relatively late are affected most. It is known that, during normal development, myelination starts in the posterior parts of the brain and spreads to anterior regions. In prefrontal regions myelination even continues into adolescence (Asato et al., 2010; Fuster, 2008). Hence it might be the case that 
this late myelination is more disturbed. Future studies to understand the timing and precise mechanisms of the damage are warranted.

\section{Conclusion}

We demonstrated extensive white matter abnormalities in patients with classic galactosemia in both the density of the neurites and the orientation dispersion (two key contributors to standard FA). Specific regional profiles were found that are in general agreement with the cognitive profile in galactosemia: a leftlateralized profile in ODI is in line with language and motor abnormalities, while the anterior pattern of NDI changes is in accordance with the general profile of higher order cognitive impairments. In addition, white matter properties were correlated with disease variables such as age and age of onset of the diet, and with behavioural outcome such as visual working memory, attention, and language performance. Hence this explorative study provides the first quantitative evidence of white matter abnormalities revealing both density and dispersion changes. Further studies should focus on specific tracts, and explore in depth the relations between these tracts and cognitive (dys)functioning.

\section{Acknowledgements}

We kindly acknowledge the Dutch Galactosemia Patient Association (GVN) for logistic support. We further thank the participants and their parents for their time, effort, cooperation and interest. The work was funded by a Galactosemia Research Fund (GOF) from the Dutch Patient Association (GVN), the School for Oncology and Development Biology (GROW), and a Maastricht University incentive (Mobilizing Minds: "Females into higher positions" to BM Jansma). 

CHAPTER 7

\section{GENERAL DISCUSSION}


$\mathrm{n}$ this dissertation, we have used a multidisciplinary approach to investigate language production impairments in patients with classic galactosemia, an inborn error of galactose metabolism (Holton et al., 2001). We aimed to learn more about the language impairments caused by this metabolic disease by using methods and perspectives from cognitive neuroscience [see Chapter 1]. The product of these joined efforts is bundled in this thesis and here I will discuss the most important results, conclusions, and further perspectives.

In the first chapters, we used electroencephalography (EEG), and its derivative event-related potentials (ERPs), to investigate temporal characteristics of language production. In particular, we adapted a paradigm in which multi-word utterances were elicited in a relatively natural manner via animated visual scenes (Indefrey et al., 2001, 2003). In the paradigm, the amount of syntactic complexity in the utterances is manipulated, allowing us to focus on syntactic planning specifically. Important to note is that experiments were conducted essentially within three groups: adolescent patients with classic galactosemia, age- and gender-matched healthy controls, and healthy adults (to obtain a basic understanding and a working model). Hence, the data presented in the different chapters are independent.

In healthy adults [Chapter 2], we observed that the paradigm was successful insofar that increasing syntactic complexity resulted in an increase in errors and corrections. Studying the ERPs (i.e., the brain's averaged response to the stimulus or task at hand), we found ERP components associated with immediate noun phrase-planning: a syntactic modulation was observed in a first P3 (450-550 ms post scene onset) and in a fronto-central negativity (600-900 ms post scene onset). In addition, we were able to examine relatively late ERP components, related to noun phrase assemblies and overall sentence integration: another P3 component varying with syntactic planning was observed (300-500 ms after a second critical visual event; equivalent to 1820$2020 \mathrm{~ms}$ post scene onset). These data shows for the first time evidence of sensitivity to syntactic planning within the P3 time window, time-locked to visual events critical of syntactic planning.

In the group of patients with classic galactosemia and matched controls [Chapter 3], the same paradigm was employed. Previous studies showed that these patients often experience speech and language impairments (see e.g., Potter et al., 2008; Waisbren et al., 1983), but the focus in the literature and clinical practice had mostly been on (motor) speech disorders. Behaviourally, we observed that the patients needed more time to prepare and finish the utterances, and made more errors. The patient ERPs had a very similar morphology to those of healthy controls, indicating overall comparable neural 
processing. However, the patients showed diverging ERP components in several time windows, starting very early in the process (90-150 ms post scene onset) and continuing throughout several language planning stages. In this study, we provided for the first time neuroscientific evidence of language production impairments in conceptual, lexical and syntactic planning stages, which could not be explained by a (motor) speech disorder only.

Comparing the adult data with the patient and adolescent control data, several agreements, but also some differences are observed. Note also that the paradigm was slightly modified (or simplified) for the adolescents by having only two syntactic conditions ('sentences' and 'words'), while the adult's paradigm contained an extra intermediate 'noun phrase' condition. Both adults and adolescents showed syntactic modulations in the behavioural data (both in the amount of corrections), in an early P3 component (adolescents: 350-650 ms post scene onset; adults: $450-550 \mathrm{~ms}$ and $600-900 \mathrm{~ms}$ post scene onset), and in a later P3 component (300-500 ms verb disambiguation in both). This comparison reveals that adults showed a more distinctive profile with two separated syntactic modulations following scene onset and more behavioural modulations. In addition, the adults showed a syntactic modulation in the voice onset times, while the adolescents did not. This indicates that in adult speakers, there is already advance syntactic planning prior to initiation of the utterance, while in adolescents this is not the case. The differences are most likely due to the age difference across the groups (adults were on average 21 years of age, while adolescents were on average 14-15 years old). In future studies, it would be interesting to draw a more direct comparison across the age groups.

In the following chapters, we used functional magnetic resonance imaging (fMRI) to investigate potential deviations in functional neural networks involved in language production. Starting with healthy adults [Chapter 4], we learned that both the left inferior frontal gyrus (IFG) and left pre-supplementary motor area (pre-SMA) show parametric modulations with syntactic planning. When looking at functional connectivity patterns, we observed that the left IFG and left preSMA communicated with a different network of brain regions during the language production. While left IFG seed was functionally correlated to a leftlateralized collection of regions in middle and inferior frontal regions, insula, planum temporale and middle temporal gyrus/superior temporal sulcus; the left pre-SMA seed was functionally connected to bilateral motor-related regions including bilateral SMA, precentral gyrus and sulcus and superior parietal regions. This study shows that the language network has specialized functional sub-networks for syntactic planning and integration on the one hand (leftlateralized network) and motor speech planning on the other hand (bilateral 
network). These functional networks may work in parallel to deal with the different aspects of language production. Future analyses will look into modulations of these correlations with syntactic planning, and into directions of connectivity (who influences who, as can be investigated using Granger Causality approaches, Roebroeck et al., 2005).

In patients with classic galactosemia [Chapter 5], we observed a generally similar network compared to the matched controls (and to the adults). All groups, including the patients, showed a syntactic modulation in left IFG. However, the patients recruited additional and more extensive frontal and motor regions while performing the language production task, and less posterior temporal regions. The patients showed an additional syntactic modulation in the left superior temporal gyrus (STG), and in right insula, and showed increased activity in the right STG and right SMA. The functional connectivity data revealed a similar pattern: a similar collection of regions was functionally correlated with the seed regions (placed in left IFG, left SMA, right insula), but in the patients more extensive connectivity with frontal and motor regions was observed, and more restricted and weaker connectivity with superior temporal regions. For the first time, we showed altered neural activity and connectivity during active language performance in classic galactosemia. The results are in agreement with the EEG/ERP study [Chapter 3], that showed increased amplitudes in several ERP components, but a generally similar morphology of the ERP waveform. The data demonstrate that language abnormalities in classic galactosemia are associated with both the cognitive counterpart of language production (including lexical and syntactic encoding), as well as the motor speech part.

Again comparing the age groups, we observed a generally similar network of regions recruited for the language production task. All three groups showed a syntactic modulation in left IFG, a region often associated with syntactic processing (e.g., Indefrey et al., 2001; Segaert et al., 2011). In contrast to the adults, however, the patients and adolescent controls did not show a syntactic modulation in left SMA. One possible methodological explanation for the absence of this effect could be the simplified version of the paradigm in the adolescents (with only two syntactic modulated conditions versus three in the adults). Another explanation could be that for adolescents the conditions were equally difficult with regard to speech preparation (i.e., age related differences in performing the task). Supporting this reasoning are a lack of syntactic modulation effects in the amount of errors and the voice onset times in adolescents, and an overall weaker performance as compared to the adults (both in accuracy and reaction times). The functional connectivity patterns were 
very similar as well, showing two separate networks correlating to left IFG and left pre-SMA. Again, a more direct comparison of these results would be interesting to investigate aspects associated with development.

Finally, in the last chapter [Chapter 6] we used diffusion weighted imaging (DWI) to assess white matter microstructure in patients with galactosemia. White matter abnormalities are often reported in classic galactosemia (through histopathological and structural imaging studies), but have never been quantified before. By using neurite orientation dispersion and density imaging (NODDI) (Zhang et al., 2012), we were able to estimate neurite density and orientation dispersion: two key contributors to fractional anisotropy (FA), the standard measure derived from diffusion tensor imaging. The data revealed extensive differences across the groups in the main white matter tracts. Specific regional patterns in density and dispersion changes were found that are in general agreement with the observed cognitive profile in galactosemia. A leftlateralized profile in dispersion changes is in line with language and motor abnormalities, while the anterior pattern of density changes is in accordance with the general profile of higher order cognitive impairments. Furthermore, several correlations were found with disease variables and behavioural outcome. Hence, this explorative study provides the first quantitative evidence of white matter microstructure abnormalities revealing both changes the density and orientation dispersion of neurites in classic galactosemia.

Taken together, we have provided evidence that patients with galactosemia show altered neural activity in conceptual, lexical and syntactic planning time windows [Chapter 3]; evidence of generally similar, but specific differences in recruitment of and connectivity between specific brain regions of interest for language planning and production [Chapter 5]; and evidence of affected white matter tracts that connect to regions involved in, among other things, language production and motor function. One of the driving forces in this dissertation was the hypothesis that motor speech disorders such as childhood apraxia of speech did not explain the full range of language production impairments that were observed in classic galactosemia [see Chapter 1]. Supporting this hypothesis are the observed impairments during language production that according to psycholinguistic theories - occur prior in time to the output stages [Chapter 3]. However, we have also provided evidence that part of the impairments is related to sensory-motor integration, motor planning and execution. In both Chapter 5 and 6, we observed altered activity in and connectivity patterns between brain areas, in addition to affected white matter tracts that are involved with both language and motor speech planning. Hence, we have to conclude that the difficulties lie not only in the cognitive part of 
language planning, and not only in motor planning or execution of speech, but in a combination of both. Future studies with designs that explicitly disentangle cognitive versus motor contributions to language production will gain more insight in their relative contributions.

Another driving force was the interesting dissociation between impaired language production and relatively intact language comprehension. Although production and comprehension have been separate fields of study in psycholinguistics, recent discussions suggest that they might be more interwoven than previously assumed, and that they largely share underlying neural mechanisms (see e.g., Kempen, 2013; Pickering and Garrod, 2013). Nevertheless, some regions and processes are more involved in production compared to comprehension. For instance, bilateral pre- and postcentral gyri showed more adaptation and hence more involvement in production than in comprehension processes (Menenti et al., 2011). In addition, the left MFG, left anterior insula, left putamen, anterior cingulate, (pre)SMA, motor cortex, and cerebellum are found to be more specifically activated during speaking than during listening (see Price, 2010; 2012, for an overview). The finding that many of these regions are specifically shown to be deviating in terms of activity or connectivity in classic galactosemia is key to explaining the observed dissociation between speaking and listening. It would be interesting to conduct specific studies into the comprehension counterpart. For instance, the same paradigm as was used in this thesis could be applied in a comprehension context (see Indefrey et al., 2003), after which direct comparisons of both tasks can be made within individuals and across groups.

An important issue in clinical studies is possible confounding by abnormal cerebral blood flow (CBF) that can contribute to an abnormal fMRI signal - the blood oxygenation level dependent (BOLD) signal (Uludağ et al., 2006). Observed group differences could well be due to differences in overall blood flow between groups and hence may lead to different sensitivity of the BOLD signal. In this thesis, we employed arterial spin labelling (ASL) to quantify CBF to examine whether patients differ in baseline CBF as compared to controls. In one region (and trends in the other regions), higher CBF was observed in the patient group. However, higher baseline CBF implies higher blood oxygenation and lower amount of deoxygenated haemoglobin. As the BOLD signal is sensitive to changes in deoxygenated haemoglobin, this will be reflected in a reduced dynamic range and hence lower BOLD signal sensitivity (i.e., lower signal change for the same amount of neuronal activation) (see e.g., Brown et al., 2003). As we observed increased BOLD signal changes in the patients, this means that these differences cannot be explained by CBF differences across 
groups. Further investigation of the baseline CBF levels in these patients, however, is warranted.

An important point to note about the fMRI studies described in this thesis is that analyses are focused on the cerebral cortex. As a method of choice, cortexbased alignment was applied to achieve an optimal alignment of the individual brains. As a consequence, subcortical structures and the cerebellum are not included in the analyses. Future studies are required to look into these structures and their involvement in classic galactosemia per se (as cerebellar involvement is often suggested in this disease, see e.g., Potter et al., 2013; Rubio-Agusti et al., 2013), and their role in the language production impairments in this disease.

Further, one obvious aim should be to be able to make predictions and statements on the individual level of the patient, both with respect to present and future performance (progression of the disease). Can we predict from the brain data which patient will show more difficulties in the cognitive planning part and less in the articulatory part, or vice versa? And what other variables (e.g., gender, age, diet, social factors) are important and can help to predict outcome? Such information will be particularly useful in designing tailored treatment approaches.

In addition to the need for more studies on language abnormalities, other cognitive domains deserve more attention as well. For instance, how can we explain that almost all patients with classic galactosemia experience difficulties with mathematics and arithmetics (Antshell et al., 2004; Bosch et al., 2004)? And what is the role of working memory in the cognitive impairments? In the EEG/ERP study [Chapter 3], we failed to find a correlation between verbal working memory performance and the ERP results, suggesting that potential impairments in verbal working memory did not contribute to the observed effects in that study. However, the important role of memory deserves more attention in this group of patient as clearly, any working memory impairments will affect the higher order cognition.

Besides using cognitive tasks in combination with neuroimaging (e.g., ERP or $\mathrm{fMRI}$ ), or correlating cognitive performance with imaging data (e.g., with density measures in white matter tracts), one could consider resting state fMRI. Resting state fMRI concerns spontaneous neural activity in the absence of any explicit task. The rationale is that these spontaneous activity patterns reveal information about the functional organization of the brain (Damoiseaux et al., 2006). The fact that there is no need for active task performance is especially attractive in studying clinical populations, where participants often have difficulties performing a task or the risk of large task-related movement effects is present. 
In galactosemia, resting state fMRI could give an overview of potential deviations in intrinsic functional networks. For instance, researchers have identified several resting state networks including the default mode, sensory/motor, executive control, fronto-parietal, and auditory network (Rosazza and Minati, 2011). It would be interesting to study these networks in patients with classic galactosemia, after which more detailed studies into the networks can follow.

Recently, researchers, clinicians and dieticians have joined forces and initiated an international consortium (European-America Galactosemia Network), supported by the patient organisations. By working together, researchers will be able to generate large sample sizes and collect a multitude of data. One of the most important aims for the consortium and for galactosemia research in general is to better understand pathophysiological mechanisms behind the complications, and to design new treatment approaches. Despite the diet, the majority of patients still develop complications mainly affecting the female gonads and the brain. Suggested mechanisms behind the brain abnormalities are: 1) glycosylation deficits affecting galactocerebrosides, which result in secondary myelin abnormalities; 2) (myo)inostitol deficiency affecting neuronal signalling. The data presented in this dissertation support the idea of abnormal myelin, resulting in white matter abnormalities [Chapter 6]. Whether the altered neural activation patterns are a result of these myelin abnormalities, a result of signalling deficiencies, or both, cannot be directly answered. For instance, it is very plausible that a more extensive recruitment of brain areas is part of an adaptation mechanism, to compensate for less efficient information transfer. And compensation might partly be quite successful, as comprehension of language is relatively intact in this disease, but it partly also fails given the observed impairments in production of language. Our discovery of specific involvement of brain areas that are particularly associated to speaking (and less or not to listening) might shed some brighter light onto the correlation of brain damage/alterations and cognitive impairments. In line with the compensation hypothesis, we see increased dispersion in white matter tracts associated with language and motor function. Increased dispersion reflects less coherent neurites, which could theoretically result in more widespread neural activity patterns. But, as the dispersion changes are rather subtle, the changes most likely only reflect local changes in microstructure, which probably would not result in the observed BOLD signal changes. Further and direct comparisons of fMRI data on cognitive functioning and NODDI data are needed to understand their link. For instance, tractography algorithms could be used to examine the microstructure of white matter tracts that connect specific regions of interest (Bastiani et al., 2012), and to investigate potential differences in terminations of 
these tracts across groups (e.g., in human arcuate fasciculus, there are more terminations in MTG compared to monkeys, and more in left hemisphere than right hemisphere, indicating involvement in language processes, Rilling et al., 2008).

Furthermore, to gain more insight in the mechanisms and the compensation theories, we would need more information about the timing of damage (e.g., prenatal, perinatal, or chronic toxicity), and about the developmental aspects. One of the drawbacks in classic galactosemia research has been the lack of an animal model showing the human phenotype. Recently, a Drosophila Melanogaster has been developed for galactosemia that has shown to be very useful to study various aspect of this disease (Kushner et al., 2010). However, this model is not suitable to study complex glycosylation abnormalities and in particular galactosylation, because of the differences with the glycosylation machinery in humans. Currently, the galactosemia research group at the Maastricht University Medical Center is working on a zebrafish (Danio Rerio) knock-out model for galactosemia. This zebrafish has shown to be an excellent model for numerous diseases affecting the brain (see e.g., Avila et al., 2007), among other things because of its transparent embryos and the rapid development. The brain and its development can be studied using imaging methods (e.g., MRI), behavioural studies (e.g., activity levels), and histological techniques (e.g., staining of myelin and neurons). Animal models should be transferred more systematically into human brain research to interpret observed patterns in (f)MRI, DWI, and ERPs. A translational approach from animal to human brain models will allow us at one point to resolve questions that have risen or remained unanswered from this dissertation and other research, and vice versa.

\section{Conclusions and take home messages}

In the current dissertation, we pioneered the application of cognitive neuroscience research in the galactosemia field. We investigated neural correlates of language impairments in classic galactosemia, using a variety of methods from cognitive neuroscience. We found evidence of impairment in the cognitive planning of language, but also in motor speech planning and execution. These impairments where reported within a temporal domain, which relates to millisecond time windows in which neural activation in patients differs from controls. Furthermore, these impairments were also mapped in the spatial domain, in terms of targeting specific regions of interests, functional connectivity, and anatomical connectivity. 
We have demonstrated the potential of joining forces across disciplines in order to achieve a better understanding of this disease. For practitioners, it will be relevant that we provided (additional) evidence that patients with galactosemia can experience more difficulties than purely related to articulation and planning of articulation. Our data suggest that in some individuals more attention should be given to linguistic planning stages (conceptual, lexical, syntactic encoding) in diagnostic and treatment settings. To patients and their families, we would like to say "thank you" for taking the efforts and for spending your time in our laboratories. Your data form a very important contribution to understanding how and why the observed differences in brain function appear, and how they relate to cognitive performance. At one point such findings must translate into appropriate treatment strategies. We hope that this dissertation can be one building stone for the complex puzzle called classic galactosemia. 


(a)CBF

(f)MRI

AF

ASL

ATL

BOLD

CAS

CSF

CC

DTI

DWI

EC

EEG

ERP

FA

FDR

GALT

GLM

GM

IC

ICA

ICE

IFG

ILF

MFG

MTG

NDI

NODDI

ODI

PET

POI

(pre)SMA

PT

RFX

ROI

SFG

SLF

SMG

STG (absolute) cerebral blood flow

(functional) Magnetic Resonance Imaging

arcuate fasciculus

arterial spin labelling

anterior temporal lobe

blood oxygenated level dependent

childhood apraxia of speech

cerebral spinal fluid

corpus callosum

diffusion tensor imaging

diffusion weighted imaging

external capsule

electroencephalography

event related potential

fractional anisotropy

false discovery rate

galactose-1-phosphate uridyl transferase

general linear model

grey matter

internal capsule

independent component analysis

intracranial electrophysiological

inferior frontal gyrus

inferior longitudinal fasciculus

middle frontal gyrus

middle temporal gyrus

neurite density index

neurite orientation dispersion and density imaging

orientation dispersion index

positron emission tomography

patch of interest

(pre)supplementary motor area

planum temporale

random-effects analysis

region of interest

superior frontal gyrus

superior longitudinal fasciculus

supramarginal gyrus

superior temporal gyrus 
STS

superior temporal sulcus

TST

total speech time

UF

uncinate fasciculus

VOT

voice onset time

WM

white matter 


REFERENCE LIST

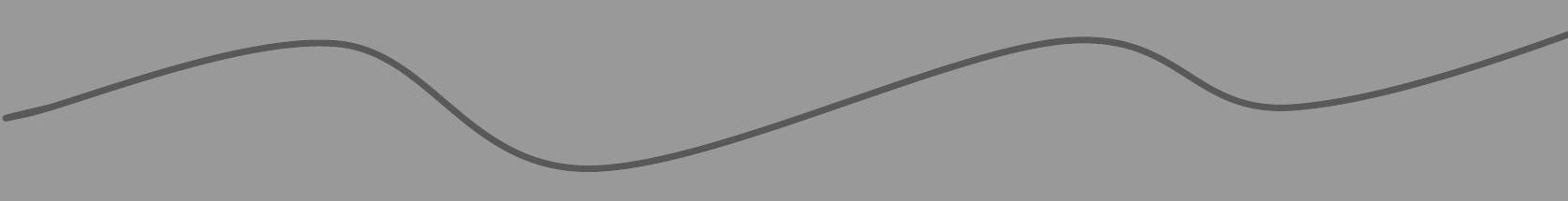


Alario, F., Costa, A., Caramazza, A., 2002. Language and Cognitive Processes Frequency effects in noun phrase production : Implications for models of lexical access. Language and Cognitive Processes. 17, 299-319.

Allum, P.H., Wheeldon, L., 2009. Scope of Lexical Access in Spoken Sentence Production: Implications for the Conceptual-Syntactic Interface. Journal of Experimental Psychology-Learning Memory and Cognition. 35, 1240-1255.

Allum, P.H., Wheeldon, L.R., 2007. Planning scope in spoken sentence production: the role of grammatical units. Journal of experimental psychology. Learning, memory, and cognition. 33, 791-810.

Andersson, J.L.R., Skare, S., Ashburner, J., 2003. How to correct susceptibility distortions in spin-echo echo-planar images: application to diffusion tensor imaging. Neuroimage. 20, 870-888.

Antshel, K.M., Epstein, I.O., Waisbren, S.E., 2004. Cognitive strengths and weaknesses in children and adolescents homozygous for the galactosemia Q188R mutation: a descriptive study. Neuropsychology. 18, 658-64.

Asato, M.R., Terwilliger, R., Woo, J., Luna, B.s., 2010. White matter development in adolescence: a DTI study. Cerebral Cortex. 20, 2122-31.

Assaf, Y., Basser, P.J., 2005. Composite hindered and restricted model of diffusion (CHARMED) MR imaging of the human brain. Neuroimage. 27, 48-58.

Avila, R.L., Tevlin, B.R., Lees, J.P., Inouye, H., Kirschner, D.A., 2007. Myelin structure and composition in zebrafish. Neurochemical research. 32, 197-209.

Badcock, N.A., Bishop, D.V., Hardiman, M.J., Barry, J.G., Watkins, K.E., 2012. Colocalisation of abnormal brain structure and function in specific language impairment. Brain and Language. 120, 310-320.

Baddeley, A., 2000. The episodic buffer: a new component of working memory? Trends in Cognitive Sciences. 4, 417-423.

Baddeley, A., 2003. Working memory and language: an overview. Journal of Communication Disorders. 36, 189-208.

Bastiani, M., Shah, N.J., Goebel, R., Roebroeck, A., 2012. Human cortical connectome reconstruction from diffusion weighted MRI: the effect of tractography algorithm. Neurolmage. 62(3), 1732-1749.

Berry, G.T., 2011. Is prenatal myo-inositol deficiency a mechanism of CNS injury in galactosemia? Journal of Inherited Metabolic Disease. 34, 345-55.

Bles, M., Alinka, A., Jansma, B., 2007. Neural aspects of cohort-size reduction during visual gating. Brain Research. 1150, 143-154.

Bock, J.K., Irwin, D.E., Davidson, D.J., 2004. Putting first things first. In: The interface of language, vision, and action: Eye movements and the visual world. Vol., J.M. Henderson, F. Ferreira, ed.^eds. Psychology Press, New York, NY, pp. 249-278.

Bock, K., 1982. Toward a cognitive psychology of syntax: Information processing contributions to sentence formulation. Psychological Review. 89, 1-47.

Bock, K., 1995. Sentence Production: From Mind to Mouth. In: Speech, Language, and Communication. Handbook of perception and cognition. Vol. 11, J.L. Miller, P.D. Eimas, ed.^eds. Academic Press, San Diego, CA, US, pp. 181-216.

Bock, K., Levelt, W., 1994. Language Production: Grammatical Encoding. In: Handbook of Psycholinguists. Vol., M.A. Gernsbacher, ed.^eds. Academic Press, San Diego, pp. 945-984.

Böhles, H., Wenzel, D., Shin, Y.S., 1986. Progressive cerebellar and extrapyramidal motor disturbances in galactosaemic twins. European journal of pediatrics. 145, 413-7. 
Bosch, A.M., Grootenhuis, M.A., Bakker, H.D., Heijmans, H.S., Wijburg, F.A., Last, B.F., 2004. Living with classical galactosemia: health-related quality of life consequences. Pediatrics. 113, e423-8.

Bouchard, K.E., Mesgarani, N., Johnson, K., Chang, E.F., 2013. Functional organization of human sensorimotor cortex for speech articulation. Nature (London). 495, 327332.

Breman, H., Peters, J., Ashburner, J., Weiskopf, W., Goebel, R., 2009. Fast fieldmapbased epi disortion correction with anatabacus: a plugin for brainvoyager. Neurolmage. 47(S1).

Brennan, J., Nir, Y., Hasson, U., Malach, R., Heeger, D.J., Pylkkänen, L., 2012. Syntactic structure building in the anterior temporal lobe during natural story listening. Brain and Language. 120, 163-173.

Brown, G.G., Eyler Zorrilla, L.T., Georgy, B., Kindermann, S.S., Wong, E.C., Buxton, R.B., 2003. BOLD and perfusion response to finger-thumb apposition after acetazolamide administration: differential relationship to global perfusion. Journal of Cerebral Blood Flow \& Metabolism. 23, 829-837.

Brown, S., Laird, A.R., Pfordresher, P.Q., Thelen, S.M., Turkeltaub, P., Liotti, M., 2009. The somatotopy of speech: Phonation and articulation in the human motor cortex. Brain and Cognition. 70, 31-41.

Brown, S., Ngan, E., Liotti, M., 2008. A larynx area in the human motor cortex. Cerebral Cortex. 18, 837-845.

Buchsbaum, B.R., Hickok, G., Humphries, C., 2001. Role of left posterior superior temporal gyrus in phonological processing for speech perception and production. Cognitive Science. 25, 663-678.

Cabeza, R., Dolcos, F., Graham, R., Nyberg, L., 2002. Similarities and differences in the neural correlates of episodic memory retrieval and working memory. Neuroimage. 16, 317-330.

Cabeza, R., Nyberg, L., 2000. Imaging cognition II: An empirical review of 275 PET and fMRI studies. Journal of Cognitive Neuroscience. 12, 1-47.

Cao, L.H., Yu, W.B., Wu, Y.H., Yu, L., 2009. The evolution, complex structures and function of septin proteins. Cellular and Molecular Life Sciences. 66, 3309-3323.

Cappa, S.F., 2012. Imaging semantics and syntax. Neuroimage. 61, 427-431.

Catani, M., Mesulam, M., 2008. The arcuate fasciculus and the disconnection theme in language and aphasia: History and current state. Cortex. 44, 953-961.

Cauda, F., D'Agata, F., Sacco, K., Duca, S., Geminiani, G., Vercelli, A., 2011. Functional connectivity of the insula in the resting brain. Neurolmage. 55, 8-23.

Çavuşoğlu, M., Pfeuffer, J., Uğurbil, K., Uludağ, K., 2009. Comparison of pulsed arterial spin labeling encoding schemes and absolute perfusion quantification. Magnetic Resonance Imaging. 27, 1039-1045.

Chapman, M.C., Jelsone-Swain, L., Johnson, T.D., Gruis, K.L., Welsh, R.C., 2013. Diffusion tensor MRI of the corpus callosum in amyotrophic lateral sclerosis. Journal of Magnetic Resonance Imaging. doi: 10.1002/jmri.24218.

Chen, J.J., Wieckowska, M., Meyer, E., Pike, G.B., 2008. Cerebral Blood Flow Measurement Using fMRI and PET: A Cross-Validation Study. International Journal of Biomedical Imaging. doi:10.1155/2008/516359

Christoffels, I.K., van de Ven, V., Waldorp, L.J., Formisano, E., Schiller, N.O., 2011. The sensory consequences of speaking: parametric neural cancellation during speech in auditory cortex. PLoS ONE. 6, e18307. 
Christoffels, I.K., Formisano, E., Schiller, N.O., 2007. Neural correlates of verbal feedback processing: an fMRI study employing overt speech. Human Brain Mapping. 28, 868-879.

Coles, M.G.H., Rugg, M.D., 1995. Event-related brain potentials: an introduction. In: Electrophysiology of Mind: Event-Related Brain Potentials and Cognition. Vol., M. Rugg, M. Coles, ed.^eds. Oxford University Press, New York, US, pp. 1-26.

Coman, D.J., Murray, D.W., Byrne, J.C., Rudd, P.M., Bagaglia, P.M., Doran, P.D., Treacy, E.P., 2010. Galactosemia, a Single Gene Disorder With Epigenetic Consequences. Pediatric Research. 67, 286-292.

Costa, A., Strijkers, K., Martin, C., Thierry, G., 2009. The time course of word retrieval revealed by event-related brain potentials during overt speech. Proceedings of the National Academy of Sciences of the United States of America. 106, 21442-21446.

Crome, L., 1962. A Case of Galactosaemia with the Pathological and Neuropathological Findings. Archives of Disease in Childhood. 37, 415-421.

de Zubicaray, G.I., McMahon, K.L., Eastburn, M.M., Pringle, A., 2006. Top-down influences on lexical selection during spoken word production: A 4T fMRI investigation of refractory effects in picture naming Human Brain Mapping. 27, 864873.

de Zubicaray, G.I., McMahon, K.L., Eastburn, M.M., Wilson, S.J., 2002. Orthographic/Phonological Facilitation of Naming Responses in the Picture-Word Task: An Event-Related fMRI Study Using Overt Vocal Responding. Neurolmage. 16, 1084-1093.

de Zubicaray, G.I., Wilson, S.J., McMahon, K.L., Muthiah, S., 2001. The Semantic Interference Effect in the Picture-Word Paradigm: An Event-Related fMRI Study Employing Overt Responses. Human Brain Mapping. 14, 218-227.

Delorme, A., Makeig, S., 2004. EEGLAB: an open source toolbox for analysis of singletrial EEG dynamics. Journal of Neuroscience Methods. 134, 9-21.

Doyle, C.M., Channon, S., Orlowska, D., Lee, P.J., 2010. The neuropsychological profile of galactosaemia. Journal of Inherited Metabolic Disease. 33, 603-9.

Dubroff, J.G., Ficicioglu, C., Segal, S., Wintering, N.A., Alavi, A., Newberg, A.B., 2008. FDG-PET findings in patients with galactosaemia. Journal of Inherited Metabolic Disease. 31, 533-539.

Elman, J.L., 1991. Distributed Representations, Simple Recurrent Networks, and Grammatical Structure. Machine Learning. 7, 195-225.

Elman, J.L., 2009. On the meaning of words and dinosaur bones: Lexical knowledge without a lexicon. Cognitive Science. 33, 547-582.

Enard, W., Przeworski, M., Fisher, S.E., Lai, C.S.L., Wiebe, V., Kitano, T., Monaco, A.P., Paabo, S., 2002. Molecular evolution of FOXP2, a gene involved in speech and language. Nature. 418, 869-872.

Evanglou, N., Esiri, M.M., Smith, S., Palace, J., Matthews, P.M., 2000. Quantitative pathological evidence for axonal loss in normal appearing white matter in multiple sclerosis. Ann. Neurol. 47, 391-395.

Ferreira, F., 1991. Effects of Length and Syntactic Complexity Prepared Utterances on initiation Times for. Journal of Memory and Language. 210-233.

Fisher, S.E., Scharff, C., 2009. FOXP2 as a molecular window into speech and language. Trends in Genetics. 25, 166-177.

Friederici, A.D., 2002. Towards a neural basis of auditory sentence processing. Trends in Cognitive Sciences. 6, 78-84. 
Friederici, A.D., 2006. The Neural Basis of Language Development and Its Impairment. Neuron. 52, 941-952.

Friederici, A.D., 2009. Pathways to language: fiber tracts in the human brain. Trends in Cognitive Sciences. 13, 175-181.

Friederici, A.D., 2011. The brain basis of language processing: from structure to function. Physiological reviews. 91, 1357-92.

Friston, K.J., Buchel, C., Fink, G.R., Morris, J., Rolls, E., Dolan, R., 1997. Psychophysiological and Modulatory Interactions in Neuroimaging. Neuroimage. 6, 218-229.

Fuster, J.M., 2008. The Prefrontal Cortex (Fourth Edition), Vol., Academic Press, London.

Gahl, S., Yu, A.C.L., 2006. Introduction to the special issue on exemplar-based models in linguistics. The Linguistic Review. 23, 213-216.

Ganushchak, L.Y., Christoffels, I.K., Schiller, N.O., 2011. The use of electroencephalography in language production research: a review. Frontiers in psychology. 2, 208. doi:10.3389/fpsyg.2011.00208

Ge, H., Yin, X., Xu, J., Tang, Y., Han, Y., Xu, W., Pang, Z., Meng, H., Liu, S., 2013. Fiber Pathways of Attention Subnetworks Revealed with Tract-Based Spatial Statistics (TBSS) and Probabilistic Tractography. PLoS ONE. 8, e78831.

Glaser, W.R., Düngelhoff, F., 1984. The time course of picture-word interference. Journal of Experimental Psychology: Human Perception and Performance. 10, 640-654.

Glasser, M.F., Rilling, J.K., 2008. DTI tractography of the human brain's language pathways. Cerebral cortex. 18, 2471-82.

Goebel, R., Esposito, F., Formisano, E., 2006. Analysis of functional image analysis contest (FIAC) data with Brainvoyager QX: From single-subject to cortically aligned group general linear model analysis and self-organizing group independent component analysis. Human Brain Mapping. 27, 392-401.

Gotts, S.J., Joon Jo, H., Wallace, G.L., Saad, Z.S., Cox, R., Martin, A., 2013. Two distinct forms of functional lateralization in the human brain. PNAS. doi/10.1073/pnas.1302581110.

Grodzinsky, Y., Friederici, A.D., 2006. Neuroimaging of syntax and syntactic processing. Current Opinion in Neurobiology. 16, 240-246.

Gubbels, C.S., Land, J.A., Rubio-Gozalbo, M.E., 2008. Fertility and Impact of Pregnancies on the Mother and Child in Classic Galactosemia. Obstetrical \& Gynecological Survey. 63, 334-343.

Gujar, S.K., Maheshwari, S., Bjorkman-Burtscher, I., Sundgren, P.C., 2005. Magnetic resonance spectroscopy. Journal of Neuro-Ophthalmology. 25, 217-26.

Haberland, C., Hof, E.G., Perou, H., Brunngraber, M., 1971. The neuropathology of galactosemia. A histopathological and biochemical study. Journal of Neuropathology \& Experimental Neurology. 30, 431-47.

Hagoort, P., 2003. How the brain solves the binding problem for language: a neurocomputational model of syntactic processing. Neuroimage. 20, S18-S29.

Hagoort, P., 2005. On Broca, brain, and binding: a new framework. Trends in Cognitive Sciences. 9, 416-423.

Hagoort, P., Brown, C., Groothusen, J., 1993. The syntactic positive shift (SPS) as an ERP measure of syntactic processing. Language and Cognitive Processes. 8, 439483.

Haller, S., Radue, E.W., Erb, M., Grodd, W., Kircher, T., 2005. Overt sentence production in event-related fMRI. Neuropsychologia. 43, 807-814. 
Harvey, P., Fossati, P., Pochon, J., Levy, R., LeBastard, G., Lehéricy, S., Allilaire, J., Dubois, B., 2005. Cognitive control and brain resources in major depression: An fMRI study using the n-back task. Neuroimage. 26, 860-869.

Hickok, G., 2009. The functional neuroanatomy of language. Phys Lif Rev. 6, 121-143.

Hickok, G., Okada, K., Serences, T., 2009. Area Spt in the human planum temporale supports sensory-motor integration for speech processing. Journal of Neuropshyiology. 101, 2725-2732.

Hickok, G., Poeppel, D., 2007. The cortical organization of speech processing. Nature reviews Neuroscience. 8, 393-402.

Hilger, I., Trost, R., Reichenbach, J.R., Linß, W., Lisy, M., Berndt, A., Kaiser, W.A., 2007. MR imaging of Her-2/neu protein using magnetic nanoparticles. Nanotechnology. 18, 135103.

Hillyard, S.A., Teder-Salejarvi, W.A., Munte, T.F., 1998. Temporal dynamics of early perceptual processing. Current Opinion in Neurobiology. 8, 202-210.

Hirschfeld, G., Jansma, B., Bolte, J., Zwitserlood, P., 2008. Interference and facilitation in overt speech production investigated with event-related potentials. Neuroreport. 19, 1227-1230.

Holton, J.B., Walter, J.H., Tyfield, L.A., 2001. Galactosemia. In: The metabolic \& molecular bases of inherited disease, C.R. Scriver, B. Childs, eds. McGraw-Hill, New York, pp. 1553-1587.

Huettel, S.A., Song, A.W., McCarthy, G., 2004. Functional Magnetic Resonance Imaging (1st edition), Vol., Sinauer Associates, Sunderland.

Hughes, J., Ryan, S., Lambert, D., Geoghegan, O., Clark, A., Rogers, Y., Hendroff, U., Monavari, A., Twomey, E., Treacy, E.P., 2009. Outcomes of Siblings with Classical Galactosemia. Journal of Pediatrics. 154, 721-726.

Indefrey, P., 2011. The spatial and temporal signatures of word production components: a critical update. Frontiers in Psychology. 2, doi: 10.3389/fpsyg.2011.00255.

Indefrey, P., Brown, C.M., Hellwig, F., Amunts, K., Herzog, H., Seitz, R.J., Hagoort, P., 2001. A neural correlate of syntactic encoding during speech production. Proceedings of the National Academy of Sciences of the United States of America. 98, 5933-6.

Indefrey, P., Hellwig, F., Herzog, H., Seitz, R.J., Hagoort, P., 2004. Neural responses to the production and comprehension of syntax in identical utterances. Brain and Language. 89, 312-319.

Indefrey, P., Levelt, W.J.M., 2004. The spatial and temporal signatures of word production components. Cognition. 92, 101-144.

Jansma, B.M., Rodriguez-Fornells, A., Möller, J., Münte, T.F., 2004. Electrophysiological studies of speech production. In: Multidisciplinary approaches to language production. Vol., T. Pechman, C. Habel, eds. Mouton de Gruyter, Berlin, pp. 361395.

Janssen, N., Barber, H.a., 2012. Phrase frequency effects in language production. PloS one. 7, e33202.

Jasper, H.H., 1958. The ten-twenty electrode system of the International Federation. Electroencephalography and Clinical Neurophysiology. 10, 370-375.

Jespersen, S.N., Bjarkam, C.R., Nyengaard, J.R., Chakravarty, M.M., Hansen, B., Vosegaard, T., Ostergaard, L., Yablonskiy, D.A., Chr. Nielsen, N., VestergaardPoulsen, P., 2010. Neurite density from magnetic resonance diffusion measurements at ultrahigh field: comparison with light microscopy and electron microscopy. Neuroimage. 49, 205-216. 
Jespersen, S.N., Leigland, L.A., Cornea, A., Kroenke, C.D., 2012. Determination of axonal and dendritic orientation distributions within the developing cerebral cortex by diffusion tensor imaging. IEEE Trans. Med. Imaging. 31, 16-32.

Jezzard, P., Balaban, B.S., 1995. Correction for geometric distortion in echo planar images from b0 field variations. MRM. 34, 65-73.

Kaan, E., Harris, A., Gibson, E., Holcomb, P., 2000. The P600 as an index of syntactic integration difficulty. Language and Cognitive Processes. 15, 159-201.

Kaufman, F.R., McBride Chang, C., Manis, F.R., Wolff, J.A., Nelson, M.D., 1995. Cognitive functioning, neurologic status and brain imaging in classical galactosemia. European Journal of Pediatrics. 154, S2-5.

Keihaninejad, S., Zhang, H., Ryan, N.S., Malone, I.B., Modat, M., Cardoso, M.J., Cash, D.M., Fox, N.C., Ourselin, S., 2013. An unbiased longitudinal analysis framework for tracking white matter changes using diffusion tensor imaging with application to Alzheimer's disease. Neurolmage. 72, 153-63.

Kell, C.A., Morillon, B., Kouneiher, F., Giraud, A.L., 2011. Lateralization of speech production starts in sensory cortices--a possible sensory origin of cerebral left dominance for speech. Cerebral cortex. 21, 932-7.

Kempen, G., 2013. Prolegomena to a Neurocomputational Architecture for Human Grammatical Encoding and Decoding. Neuroinformatics.

Klingberg, T., 2006. Development of a superior frontal-intraparietal network for visuospatial working memory. Neuropsychologia. 44, 2171-2177.

Koester, D., Schiller, N.O., 2008. Morphological priming in overt language production: Electrophysiological evidence from Dutch. Neuroimage. 42, 1622-1630.

Kok, A., 2001. On the utility of P3 amplitude as a measure of processing capacity. Psychophysiology. 38, 557-577.

Konopka, A.E., 2012. Planning ahead: How recent experience with structures and words changes the scope of linguistic planning. Journal of Memory and Language. 66, 143-162.

Krabbi, K., Uudelepp, M.-L., Joost, K., Zordania, R., Õunap, K., 2011. Long-term complications in Estonian galactosemia patients with a less strict lactose-free diet and metabolic control. Molecular genetics and metabolism. 103, 249-53.

Kutas, M., Schmitt, B.M., 2003. Language in Microvolts. In: Mind, brain, and language: Multidisciplinary perspectives. Vol., M.T. Banich, M. Mack, eds. Lawrence Erlbaum Associates, NJ, pp. 171-209.

Langacker, R.W., 1987. Foundations of cognitive grammar: Theoretical prerequisites, Vol., Stanford Univ Press, Stanford.

Lebea, P.J., Pretorius, P.J., 2005. The molecular relationship between deficient UDPgalactose uridyl transferase (GALT) and ceramide galactosyltransferase (CGT) enzyme function: a possible cause for poor long-term prognosis in classic galactosemia. Medical Hypotheses. 65, 1051-7.

Levelt, W.J.M., 1989. Speaking: From Intention to Articulation, Vol., The MIT Press, Cambridge, MA.

Levelt, W.J.M., Roelofs, A.S., Meyer, A., 1999. A theory of lexical access in speech production. Behavioral and Brain Sciences. 22, 1-75.

Lewis, F.M., Coman, D.J., Kilcoyne, S., Murdoch, B.E., Syrmis, M., 2013. Pre-linguistic communication skill development in an infant with a diagnosis of galactosaemia. Developmental neurorehabilitation. 1-7. 
Lewis, F.M., Coman, D.J., Syrmis, M., Kilcoyne, S., Murdoch, B.E., 2012. Differential Phonological Awareness Skills in Children with Classic Galactosemia: A Descriptive Study of Four Cases. JIMD reports. 10, 45-52.

Lezak, M.D., 1995. Neuropsychological Assessment, 4th edition, Vol., Oxford University Press, New York, NY.

Li, K., Guo, L., Nie, J., Li, G., Liu, T., 2009. Review of methods for functional brain connectivity detection using fMRI. Computerized Medical Imaging and Graphics. 33, 131-139.

Liegeois, F., Baldeweg, T., Connolly, A., Gadian, D.G., Mishkin, M., Vargha-Khadem, F., 2003. Language fMRI abnormalities associated with FOXP2 gene mutation. Nature Neuroscience. 6, 1230-1237.

Luck, S.J., 2005. An Introduction to the Event-Related Potential Technique, Vol., MIT Press.

Makeig, S., Onton, J., 2009. ERP features and EEG dynamics: an ICA perspective. In: Oxford Handbook of Event-Related Potential Components. Vol., S.J. Luck, E. Kappenman, eds. Oxford University Press, New York.

Marek, A., Habets, B., Jansma, B.M., Nager, W., Münte, T.F., 2007. Neural correlates of conceptualisation difficulty during the preparation of complex utterances. Aphasiology. 21, 1147-1156.

Martin, A., Chao, L.L., 2001. Semantic memory and the brain: structure and processes. Current Opinion in Neurobiology. 11, 194-201.

Menenti, L., Gierhan, S.M.E., Segaert, K., Hagoort, P., 2011. Shared Language: Overlap and Segregation of the Neuronal Infrastructure for Speaking and Listening Revealed by Functional MRI. Psychological Science. doi: 10.1177/0956797611418347.

Mercier, M., Schwartz, S., Michel, C.M., Blanke, O., 2009. Motion direction tuning in human visual cortex. European Journal of Neuroscience. 29, 424-434.

Meyer, A.S., 1992. Investigation of Phonological Encoding through Speech Error Analyses - Achievements, Limitations, and Alternatives. Cognition. 42, 181-211.

Meyer, A.S., 1996. Lexical access in phrase and sentence production. Journal of Memory and Language. 35, 477-496.

Meyer, A.S., Lethaus, F., 2004. The use of eye tracking in studies of sentence generation. In: The interface of language, vision, and action: What we can learn from free-viewing eye tracking. Vol., J.M. Henderson, F. Ferreira, eds. Psychology Press, New York, pp. 191-211.

Meyers, J.E., Meyers, K.R., 1995. Rey Complex Figure Test and Recognition Trial: Professional Manual, Vol., Psychological Assessment Resources, Inc., Odessa.

Moore, W.H., 1989. Language recovery in aphasia: A right hemisphere perspective. Aphasiology. 3, 101-110.

Mortensen, L., Meyer, A.S., Humphreys, G.W., 2008. Speech planning during multipleobject naming: Effects of ageing. The Quarterly Journal of Experimental Psychology. 61, 1217-1238.

Munte, T.F., Schiltz, K., Kutas, M., 1998. When temporal terms belie conceptual order. Nature. 395, 71-73.

Nachev, P., Kennard, C., Husain, M., 2008. Functional role of the supplementary and pre-supplementary motor areas. Nature Reviews Neuroscience. 9, 856-869.

Nelson, C.D., Waggoner, D.D., Donnell, G.N., Tuereck, J.M., Buist, N.R.M., 1991. Verbal Dyspraxia in Treated Galactosemia. Pediatrics. 88, 349-350. 
Nelson, M.D., Wolff, J.A., Cross, C.A., Donnell, G.N., Kaufman, F.R., 1992. Galactosemia: Evaluation with MR Imaging. Radiology. 184, 255-261.

Ng, W.G., Xu, Y.K., Kaufman, F.R., Donnell, G.N., 1989. Deficit of uridine diphosphate galactose in galactosaemia. J Inherit Metab Dis. 12, 257-266.

Nierenberg, J., Pomara, N., Hoptman, M.J., Sidtis, J.J., Ardekani, B.A., Lim, K.O., 2005. Abnormal white matter integrity in healthy apolipoprotein $\mathrm{E}$ epsilon4 carriers. Neuroreport. 16, 1369-1372.

Ogar, J., Slama, H., Dronkers, N., Amici, S., Gorno Tempini, M.L., 2005. Apraxia of speech: an overview. Neurocase: The neural basis of cognition. 11, 427-432.

Onton, J., Westerfield, M., Townsend, J., Makeig, S., 2006. Imaging human EEG dynamics using independent comonent analysis. Neuroscience and Biobehavioral Reviews. 30, 808-822.

Osterhout, L., Holcomb, P.J., 1992. Event-Related Brain Potentials Elicited by Syntactic Anomaly. Journal of Memory and Language. 31, 785-806.

Otaduy, M.C.G., Leite, C.C., Lacerda, M.T.C., Costa, M.O.R., Arita, F., Prado, E., Rosemberg, S., 2006. Proton MR spectroscopy and imaging of a galactosemic patient before and after dietary treatment. Am J Neuroradiol. 27, 204-207.

Panis, B., Forget, P.P., van Kroonenburgh, M.J., Vermeer, C., Menheere, P.P., Nieman, F.H., Rubio Gozalbo, M.E., 2004. Bone metabolism in galactosemia. Bone. 35, 9827.

Pickering, M.J., Branigan, H.P., 1999. Syntactic priming in language production. Trends in cognitive sciences. 3, 136-141.

Pickering, M.J., Garrod, S., 2013. How tightly are production and comprehension interwoven? Frontiers in psychology. 4, 238.

Pierpaoli, C., Jezzard, P., Basser, P.J., Barnett, A., Chiro, G.D., 1996. Diffusion tensor MR imaging of the human brain. Radiology. 201, 637-648.

Potter, N.L., 2011. Voice disorders in children with classic galactosemia. Journal of Inherited Metabolic Disease. 34, 377-85.

Potter, N.L., Lazarus, J.A.C., Johnson, J.M., Steiner, R.D., Shriberg, L.D., 2008. Correlates of language impairment in children with galactosaemia. Journal of Inherited Metabolic Disease. 31, 524-32.

Potter NL, Nievergelt Y, Shriberg LD (2013) Motor and Speech Disorders in Classic Galactosemia. JIMD reports 11: 31-41.

Price, C.J., 2010. The anatomy of language: a review of $100 \mathrm{fMRI}$ studies published in 2009. Cognitive Neuroscience. 1191, 62-88.

Price, C.J., 2012. A review and synthesis of the first 20 years of PET and fMRI studies of heard speech, spoken language and reading. Neurolmage. 62, 816-47.

Price, C.J., Friston, K.J., 1997. The temporal dynamics of reading: a PET study. Proceedings. Biological sciences / The Royal Society. 264, 1785-91.

Rabovsky, M., Sommer, W., Rahman, R.A., 2012. Depth of Conceptual Knowledge Modulates Visual Processes during Word Reading. Journal of Cognitive Neuroscience. 24(4), 990-1005.

Rahman, R.A., Sommer, W., 2008. Seeing what we know and understand: How knowledge shapes perception. Psychonomic Bulletin \& Review. 15, 1055-1063.

Rahman, R.A., van Turennout, M., Levelt, W.J.M., 2003. Phonological encoding is not contingent on semantic feature retrieval: An electrophysiological study on object naming. Journal of Experimental Psychology-Learning Memory and Cognition. 29, 850-860. 
Reading, S.A., Yassa, M.A., Bakker, A., Dziorny, A., Gourley, L., Yallapragada, V., Rosenblatt, A., Margolis, R., Aylward, E., Brandt, J., 2005. Regional white matter change in pre-symptomatic Huntington's disease: a diffusion tensor imaging study. Psychiatry Research. 140, 55-62.

Riecker, A., Mathiak, K., Wildgruber, D., Erb, M., Hertrich, I., Grodd, W., Ackermann, H., 2005. fMRI reveals two distinct cerebral networks subserving speech motor control. Neurology. 64, 700-706.

Rilling, J.K., Glasser, M.F., Preuss, T.M., Ma, X.Y., Zhao, T.J., Hu, X.P., Behrens, T.E.J., 2008. The evolution of the arcuate fasciculus revealed with comparative DTI. Nature Neuroscience. 11, 426-428.

Robertson, A., Singh, R.H., Guerrero, N.V., Hundley, M., Elsas, L.J., 2000. Outcomes analysis of verbal dyspraxia in classic galactosemia. Genetics in Medicine. 2, 142148.

Roebroeck, A., Formisano, E., Goebel, R., 2005. Mapping directed influence over the brain using Granger causality and fMRI. Neuroimage. 25, 230-42.

Rogalski, E., Cobia, D., Harrison, T.M., Wieneke, C., Thompson, C.K., Weintraub, S., Mesulam, M.M., 2011. Anatomy of Language Impairments in Primary Progressive Aphasia. The Journal of Neuroscience. 31, 3344-3350.

Roosendaal, S.D., Geurts, J.J.G., Vrenken, H., Hulst, H.E., Cover, K.S., Castelijns, J.A., Pouwels, P.J.W., Barkhof, F., 2009. Regional DTI differences in multiple sclerosis patients. Neuroimage. 44, 1397-1403.

Rosazza, C., Minati, L., 2011. Resting-state brain networks: literature review and clinical applications. Neurological sciences. 773-787.

Rubio-Agusti I, Carecchio M, Bhatia KP, et al. (2013) Movement Disorders in Adult Patients With Classical Galactosemia. Mov Disord 28(6): 804-810.

Rubio-Gozalbo, M.E., Gubbels, C.S., Bakker, J.A., Menheere, P.P.C.A., Wodzig, W.K.W.H., Land, J.A., 2010. Gonadal function in male and female patients with classic galactosemia. Human Reproduction Update. 16, 177-188.

Sahin, N.T., Pinker, S., Cash, S.S., Schomer, D., Halgren, E., 2009. Sequential Processing of Lexical, Grammatical, and Phonological Information Within Broca's Area. Science. 326, 445-449.

Sahin, N.T., Pinker, S., Halgren, E., 2006. Abstract grammatical processing of nouns and verbs in Broca's area: evidence from fMRI. Cortex. 42, 540-562.

Saur, D., Kreher, B.W., Schnell, S., Kummerer, D., Kellmeyer, P., Vry, M., Umarova, R., Musso, M., Glauche, V., Abel, S., Huber, W., Rijntjes, M., Hennig, J., Weiller, C., 2008. Ventral and dorsal pathways for language. PNAS. 105, 18035-18040.

Schadewaldt, P., Hoffman, B., Hammen, H., Kamp, G., Schweitzer-Krantz, S., Wendel, U., 2010. Longitudinal Assessment of Intellectual Achievement in Patients with Classical Galactosemia. Pediatrics. 125, e374-e381.

Schiller, N.O., Caramazza, A., 2006. Grammatical gender selection and the representation of morphemes: The production of Dutch diminutives. Language and cognitive processes. 21, 1011-1029.

Schmitt, B.M., Münte, T.F., Kutas, M., 2000. Electrophysiological estimates of the time course of semantic and phonological encoding during implicit picture naming. Psychophysiology. 37, 473-484.

Schmitt, B.M., Rodriguez-Fornells, A., Kutas, M., Münte, T.F., 2001a. Electrophysiological estimates of semantic and syntactic information access during tacit picture naming and listening to words. Neuroscience Research. 41, 293-298. 
Schmitt, B.M., Schiltz, K., Zaake, W., Kutas, M., Münte, T.F., 2001b. An electrophysiological analysis of the time course of conceptual and syntactic encoding during tacit picture naming. Journal of Cognitive Neuroscience. 13, 510522.

Schmitt, B.M., Schiltz, K., Zaake, W., Münte, T.F., Kutas, M., 2000. The time course of conceptual and syntactic encoding during language production estimated by event related potentials. Journal of Cognitive Neuroscience. 29B.

Schweitzer, S., Shin, Y., Jakobs, C., Brodehl, J., 1993. Long-term outcome in 134 patients with galactosemia. European Journal of Pediatrics. 152, 36-43.

Segaert, K., Menenti, L., Weber, K., Petersson, K.M., Hagoort, P., 2011. Shared Syntax in Language Production and Language Comprehension--An fMRI Study. Cerebral cortex. doi:10.1093/cercor/bhr249.

Shield, J.P., Wadsworth, E.J., MacDonald, A., Stephenson, A., Tyfield, L., Holton, J.B., Marlow, N., 2000. The relationship of genotype to cognitive outcome in galactosaemia. Archives of Disease in Childhood. 83, 248-50.

Shriberg, L.D., Potter, N.L., Strand, E.A., 2011. Prevalence and Phenotype of Childhood Apraxia of Speech In Youth with Galactosemia. Journal of Speech, Language, and Hearing Research. 54(2), 487-519.

Smith, S.M., Jenkinson, M., Johansen-Berg, H., Rueckert, D., Nichols, T.E., Mackay, C.E., Watkins, K.E., Ciccarelli, O., Cader, M.Z., Matthews, P.M., Behrens, T.E.J., 2006. Tract-based spatial statistics: Voxelwise analysis of multi-subject diffusion data. Neuroimage. 31, 1487-1505.

Smith, S.M., Jenkinson, M., Woolrich, M.W., Beckmann, C.F., Behrens, T.E.J., Johansen-Berg, H., Bannister, P.R., De Luca, M., Drobnjak, I., Flitney, D.E., Niazy, R., Saunders, J., Vickers, J., Zhang, Y., De Stefano, N., Brady, J.M., Matthews, P.M., 2004. Advances in functional and structural MR image analysis and implementation as FSL. Neuroimage. 23, 208-219.

Smith, S.M., Nichols, T.E., 2009. Threshold-free cluster enhancement: addressing problems of smoothing, threshold dependence and localisation in cluster inference. Neuroimage. 44, 83-98.

Snijders, T.M., Petersson, K.M., Hagoort, P., 2010. Effective connectivity of cortical and subcortical regions during unification of sentence structure. Neuroimage. 52, 16331644.

Snijders, T.M., Vosse, T., Kempen, G., Van Berkum, J., Petersson, K.M., Hagoort, P., 2009. Retrieval and unification of syntactic structure in sentence comprehension: an FMRI study using word-category ambiguity. Cerebral cortex. 19, 1493-503.

Sternberg, S., 1969. The discovery of processing stages: Extension of Donders' method. Attention and Performance II, Acta Psychologica. 30, 276-315.

Strijkers, K., Costa, A., Thierry, G., 2009. Tracking Lexical Access in Speech Production: Electrophysiological Correlates of Word Frequency and Cognate Effects. Cerebral Cortex. 20, 912-928.

Stroop, J.R., 1935. Studies of interference in serial verbal reactions. Journal of Experimental Psychology. 18, 643-662.

Timmers, I., Gentile, F., Rubio-Gozalbo, M.E., Jansma, B.M., 2013. Temporal characteristics of online syntactic sentence planning: an event-related potential study. PLoS ONE 8(12): e82884.

Timmers, I., Jansma, B.M., Rubio Gozalbo, M.E., 2012. From mind to mouth: event related potentials of sentence production in classic galactosemia. PLoS ONE. 7, e52826. 
Timmers, I., van den Hurk, J., Di Salle, F., Rubio-Gozalbo, M.E., Jansma, B.M., 2011. Language production and working memory in classic galactosemia from a cognitive neuroscience perspective: Future research directions. Journal of Inherited Metabolic Disease. 34, 367-376.

Tomasello, M., 2005. Constructing a language: A usage-based theory of language acquisition, Vol., Harvard Univ Press, Cambridge, MA.

Tremblay, P., Small, S.L., 2011. Motor response selection in overt sentence production: a functional MRI study. Frontiers in Psychology. 2, doi: 10.3389/fpsyg.2011.00253.

Uludağ, K., Dubowitz, D.J., Buxton, R.B., 2006. Basic principles of functional MRI. In: Clinical magnetic resonance imaging. Vol. 3rd edition, R.R. Edelman, J.R. Hesselink, M.B. Zlatkin, J.V. Crues, eds. Saunders Elsevier, Philadelphia, pp. 249287.

van Haasen, P.P., de Bruyn, E.E.J., Pijl, Y.L., Poortinga, Y.H., Spelberg, H.C., van den Steene, G., Coetsier, P., Spoelders-Claes, R., Stinissen, J., 1986. WISC-R, Weschler Intelligence Scale for Children - Revised, Nederlandstalige uitgave. Deel I. Testinstructie; Deel II. Scoring en Normen; Deel III. Verantwoording., Vol., Swets \& Zeitlinger, Lisse.

van Turennout, M., Hagoort, P., Brown, C.M., 1998. Brain activity during speaking: From syntax to phonology in 40 milliseconds. Science. 280, 572-574.

van Turennout, M., Hagoort, P., Brown, C.M., 1999. The time course of grammatical and phonological processing during speaking: Evidence from event-related brain potentials. Journal of Psycholinguistic Research. 28, 649-676.

Vos, P.G., 1988. Bourdon-Vos-Test [Bourdon-Vos Test], Vol., Swets \& Zeitlinger, Lisse

Vosse, T., Kempen, G., 2000. Syntactic structure assembly in human parsing: a computational model based on competitive inhibition and a lexicalist grammar. Cognition. 75, 105-43.

Vosse, T.G., Kempen, G.A.M., 2008. Parsing Verb-Final Clauses in German: Gardenpath and ERP Effects Modeled by a Parallel Dynamic Parser. In B. Love, K. McRae, \& V. Sloutsky (Eds.), Proceedings of the 30th Annual Conference on the Cognitive Science Society (pp. 261-266). Washington: Cognitive Science Society.

Waggoner, D.D., Buist, N.R.M., Donnell, G.N., 1990. Long-Term Prognosis in Galactosemia: Results of a Survey of 350 Cases. Journal of Inherited Metabolic Disease. 802-818.

Wagner, V., Jescheniak, J.D., Schriefers, H., 2010. On the flexibility of grammatical advance planning during sentence production: Effects of cognitive load on multiple lexical access. Journal of experimental psychology. Learning, memory, and cognition. 36, 423-40.

Waisbren, S.E., Norman, T.R., Schnell, R.R., Levy, H.L., 1983. Speech and language deficits in early-treated children with galactosemia. Journal of Pediatrics. 102, 75-7.

Waisbren, S.E., Potter, N.L., Gordon, C.M., Green, R.C., Greenstein, P., Gubbels, C.S., Rubio-Gozalbo, M.E., Schomer, D., Welt, C., Anastasoaie, V., D'Anna, K., Gentile, J., Guo, C.Y., Hecht, L., Jackson, R., Jansma, B.M., Li, Y.J., Lip, V., Miller, D.T., Murray, M., Power, L., Quinn, N., Rohr, F., Shen, Y.P., Skinder-Meredith, A., Timmers, I., Tunick, R., Wessel, A., Wu, B.L., Levy, H., Elsas, L., Berry, G.T., 2012. The adult galactosemic phenotype. Journal of Inherited Metabolic Disease. 35, 279286.

Wakana S, Jiang H, Nagae-Poetscher LM, van Zijl PCM, Mori S (2003) Fiber Tract based Atlas of human white matter anatomy. Radiology 230(1): 77-87. 
Wang, Z.J., Berry, G.T., Dreha, S.F., Zhao, H., Segal, S., Zimmerman, R.A., 2001. Proton magnetic resonance spectroscopy of brain metabolites in galactosemia. Ann Neurol. 50, 266-269.

Webb, A.L., Singh, R.H., Kennedy, M.J., Elsas, L.J., 2003. Verbal dyspraxia and galactosemia. Pediatric Research. 53, 396-402.

Widhalm, K., Miranda-da-Cruz, B.D., de Sonneville, L.M.J., 2002. Information processing characteristics and uridine treatment in children with classical galactosemia. Nutrition Research. 22, 257-270.

Winston, G.P., 2012. The physical and biological basis of quantitative parameters derived from diffusion MRI. Quantitative imaging in medicine and surgery. 2, 254265.

Winston, G.P., Micallef, C., Symms, M.R., Alexander, D.C., Duncan, J.S., Zhang, H., 2013. Advanced diffusion imaging sequences could aid assessing patients with focal cortical dysplasia and epilepsy. Epilepsy Research. http://dx.doi.org/10.1016/j.eplepsyres.2013.11.004.

Wood, J.N., Grafman, J., 2003. Human prefrontal cortex: processing and representational perspectives. Nature Reviews Neuroscience. 4, 139-147.

Xiang, H., Fonteijn, H.M., Norris, D.G., Hagoort, P., 2010. Topographical functional connectivity pattern in the perisylvian language networks. Cerebral cortex. 20, 549560 .

Ye, Z., Habets, B., Jansma, B.M., Münte, T.F., 2011. Neural basis of linearization in speech production. Journal of Cognitive Neuroscience. 23, 3694-3702.

Zhang, H., Schneider, T., Wheeler-Kingshott, C.a., Alexander, D.C., 2012. NODDI: Practical in vivo neurite orientation dispersion and density imaging of the human brain. Neurolmage. 61, 1000-1016.

Zhang, H., Yushkevich, P.a., Alexander, D.C., Gee, J.C., 2006. Deformable registration of diffusion tensor MR images with explicit orientation optimization. Medical image analysis. 10, 764-85. 

T he focus in this thesis is on language production impairments in classic galactosemia - an inherited disease of galactose metabolism. We examined neural correlates of these impairments experienced by patients with classic galactosemia by using methods and perspectives from cognitive neuroscience. Previous studies have examined the cognitive profile in classic galactosemia, and imaging studies have shown structural abnormalities in the brain. In this thesis we investigated the brain online - during an active language production task - to establish the link between brain function and the observed language impairments. We examined temporal (when in time) and spatial (where in the brain) aspects of language production in the healthy adult brain, and in analogy in a group of patients with classic galactosemia who were compared to age- and gender matched controls.

In Chapter 1, we introduced the disease classic galactosemia and the complications that patients with this disease can experience, despite compliance to the dietary treatment. We presented basic cognitive neuroscience techniques and cognitive models related to language production and working memory, and described how these could be applied to the study of this disease. The most relevant techniques in this thesis are: electroencephalography (EEG) and functional and diffusion weighted magnetic resonance imaging ( $\mathrm{fMRI}$ and DW-MRI, respectively). Using EEG, one is able to look into a brain process of interest at a milliseconds time scale (i.e., high temporal resolution). $\mathrm{fMRI}$ on the other hand has high spatial resolution and is able to locate networks involved in language, and to define functional connectivity among regions within the network (e.g., which brain regions are working together during language). DW$\mathrm{MRI}$ can be used to assess properties and potential abnormalities in white matter microstructure (i.e., the fibres through which information travels across brain cells or neurons). The aim of applying these methods and accompanying paradigms in galactosemia research - an approach that has never been taken before - is to gain more insight in the pathophysiological processes in galactosemia.

In Chapter 2, we studied healthy adult volunteers using electroencephalography (EEG) and its derivative event-related potentials (ERPs) to investigate the time course of syntactic planning during sentence production. During the syntactic planning stage, grammatical roles (e.g., subject versus object) and syntactical functions (e.g., tense) are assigned, and elements are assembled into a syntactic frame. To study this stage, a paradigm was used in which visual animated scenes were presented to elicit utterances that varied in syntactic complexity (from words to noun phrases to sentences) - a paradigm used throughout this thesis. In the behavioural data, we observed an increase in 
errors and corrections with increasing syntactic complexity, indicating a successful experimental manipulation. In the ERPs, we observed that the syntactic complexity variation resulted in several modulations across the entire time window. The data showed that the P300 time window is sensitive to syntactic planning, both at noun phrase-level and at sentence-level. Further, we demonstrated that overt naming can be studied in EEG/ERP using animated scenes in a relatively natural manner.

In Chapter 3, the same paradigm was applied to examine sentence production in adolescent patients with classic galactosemia versus age- and gendermatched controls. We observed that the patients needed more time to prepare and finish the utterances and made more errors. Further, the patient ERPs had a very similar morphology to that of healthy controls, indicating overall comparable neural processing. However, the ERPs also diverged from those of controls in several functionally informative time windows, ranging from very early (90-150 ms after scene onset) to relatively late (1820-2020 ms after scene onset). These time windows can be associated with different linguistic encoding stages. These results present the first neuroscientific evidence for language production impairments in patients with classic galactosemia in lexical and syntactic planning stages, i.e., prior to the linguistic output phase.

In Chapter 4, we investigated spatial aspects of language planning. Functional neural networks involved in sentence planning were examined in healthy adults. Again, a similar paradigm was used, now combined with functional magnetic resonance imaging (fMRI) and functional connectivity. These techniques allowed us to study which brain areas are activated during the task and which areas work functionally together. We observed that activity in both the left inferior frontal gyrus (IFG) and left pre-supplementary motor area (pre-SMA) increased with increasing syntactic complexity. Further, we observed that these regions correlated with different functional networks. The study showed that the language network has specialized functional sub-networks that may work in parallel to deal with different aspects of language production.

In Chapter 5, patients with classic galactosemia and matched controls were studied with fMRI while performing the same sentence production task. Results showed that the patients recruited a generally similar network of brain regions compared to healthy controls, but also additional and more extensive regions. In the functional connectivity patterns, a similar pattern emerged with more extensive connectivity with frontal and motor regions in the patients, and more restricted and weaker connectivity with superior temporal regions. The data demonstrated that language abnormalities in classic galactosemia are associated with specific changes within the language network. These changes 
point towards impairments related to both syntactic planning and motor speech planning in patients with classic galactosemia.

In Chapter 6, diffusion weighted imaging was used to investigate potential abnormalities in white matter microstructure properties in patients with classic galactosemia, as compared to matched healthy controls. We applied neurite orientation dispersion and density imaging (NODDI), a technique designed to estimate neurite density and orientation dispersion, two key contributors to the widely used fractional anisotropy (FA, derived from standard diffusion tensor imaging - DTI). Results showed extensive white matter abnormalities: neurite density index (NDI) was lower in the patient group in bilateral anterior areas, and orientation dispersion index (ODI) was increased mainly in the left hemisphere. The specific regional profiles are in general agreement with the cognitive profile observed in classic galactosemia showing higher order cognitive impairments, and language and motor impairments, respectively. Moreover, the white matter properties correlated with disease variables (i.e., age, age at onset of diet) and with behavioural outcome (e.g., visual working memory). This explorative study provides the first quantitative measures of white matter abnormalities revealing both density and dispersion changes. These findings confirm and extend previous qualitative observations of white matter abnormalities. The measured changes in microstructure are in line with abnormal myelination, although the increased neurite dispersion in orientation might partly also reflect compensation mechanisms.

Finally, Chapter 7 provides a general discussion, where the most important results and conclusions of the studies presented in this thesis are discussed. Furthermore, parallels are drawn across results and future perspectives are considered. Taken together, we pioneered the application of cognitive neuroscience research in the galactosemia field and have demonstrated the potential of joining forces across disciplines in order to achieve a better understanding of this disease. 



$$
m
$$


$D^{2}$ e focus in dit proefschrift ligt op het bestuderen van problemen met taalproductie in klassieke galactosemie - een erfelijke ziekte in de galactose-stofwisseling. We hebben de neurale correlaten onderzocht van deze problemen die patiënten met klassieke galactosemie veelal ervaren. Daarvoor hebben we gebruik gemaakt van methoden en perspectieven uit de cognitieve neurowetenschappen. Eerdere studies hebben al gekeken naar het cognitieve profiel van patiënten met klassieke galactosemie en door gebruik te maken van beeldvormende technieken zijn er ook structurele afwijkingen in de hersenen geconstateerd. In dit proefschrift was het doel om de hersenen aan het werk (online) te bestuderen - tijdens actieve uitvoering van een taalproductietaak - om de link vast te stellen tussen het functioneren van de hersenen en de geobserveerde taalproblemen. We hebben gekeken naar temporele (wanneer in tijd) en spatiële (waar in de hersenen) aspecten van taalproductie. Dit hebben we gedaan zowel in een groep gezonde volwassenen en in analogie ook in een groep patiënten met klassieke galactosemie welke vergeleken werden met een controlegroep met vergelijkbare leeftijd en geslacht.

In Hoofdstuk 1 hebben we de ziekte klassieke galactosemie geïntroduceerd alsmede de complicaties die patiënten met deze ziekte ondanks het volgen van de dieetbehandeling kunnen ervaren. We hebben elementaire technieken uit de cognitieve neurowetenschappen en cognitieve modellen met betrekking tot taalproductie en werkgeheugen gepresenteerd. De meest relevante technieken die we hebben gebruikt in dit proefschrift zijn: elektro-encefalografie (EEG), en functionele en diffusie-gewogen magnetic resonance imaging (fMRI en DW$\mathrm{MRI}$, respectievelijk). Met EEG kunnen we naar de relevante hersenprocessen kijken op een tijdsschaal van milliseconden (dus een hoge temporele resolutie). fMRI, aan de andere kant, heeft hoge spatiële resolutie en is in staat om hersennetwerken te lokaliseren die betrokken zijn bij taal. Ook kunnen we met behulp van fMRI kijken naar functionele connectiviteit tussen gebieden binnen zulke netwerken (dus welke hersengebieden samenwerken tijdens het produceren van taal bijvoorbeeld). DW-MRI wordt gebruikt om kenmerken en eventuele afwijkingen in de microstructuur van witte stof te onderzoeken (dit zijn de verbindingen tussen hersencellen ofwel de vezelbanen waar de informatie door reist). Het doel van het toepassen van deze methoden en bijbehorende paradigma's in onderzoek naar klassieke galactosemie - een aanpak die nog niet eerder is genomen - is om meer inzicht te krijgen in de pathofysiologische processen die zich afspelen in deze ziekte.

In Hoofdstuk 2 onderzochten we het tijdsverloop van syntactische planning tijdens zinproductie in gezonde volwassen vrijwilligers met behulp van EEG en de afgeleide event-related potentials (ERPs). Syntactische planning is een 
stadium in de taalplanning waarin grammaticale rollen (bijv. onderwerp versus lijdend voorwerp) en syntactische functies (bijv. de tijd) worden toegewezen en elementen worden samengevoegd in een syntactisch frame. Om dit stadium te onderzoeken, hebben we een paradigma / taak gebruikt waarin visueel geanimeerde scènes werden gepresenteerd. We vroegen de deelnemers om deze scènes te beschrijven in uitingen variërend in syntactische complexiteit (van woorden, via naamwoordgroepen [noun phrases], tot hele zinnen) - een paradigma dat vaker in dit proefschrift gebruikt wordt. In de gedragsdata zagen we een toename van fouten en correcties naarmate de syntactische complexiteit toenam. Dit wijst op een succesvolle experimentele manipulatie van syntactische complexiteit. In de ERP's zagen we dat de variatie in syntactische complexiteit resulteerde in een aantal modulaties verspreid over het gehele tijdvenster. De gegevens toonden aan dat het zogenaamde P300 tijdvenster gevoelig is voor syntactische planning, zowel op het niveau van naamwoordgroepen als op zin-niveau. Verder hebben we aangetoond dat overte taalproductie (hardop praten) kan worden bestudeerd met behulp van $E E G / E R P$ en geanimeerde scènes om een relatief natuurlijke setting te creëren.

In Hoofdstuk 3 hebben we hetzelfde paradigma toegepast om zin-productie te bestuderen in adolescente patiënten met klassieke galactosemie versus leeftijden geslacht-gematchte controles. We vonden dat de patiënten meer tijd nodig hadden voor de voorbereiding en de afwerking van de uitingen en dat ze bovendien meer fouten maakten. Verder hadden de ERP's van de patiënten een morfologie / vorm die vergelijkbaar was met die van de gezonde controles, wat wijst op een over het algemeen vergelijkbare neurale verwerking. Echter, we vonden ook dat de ERP's van de patiënten afwijkingen vertoonden vergeleken met die van de controles in verschillende tijdvensters, variërend van zeer vroege (90-150 ms na start van de scène) tot relatief late vensters (1820$2020 \mathrm{~ms}$ na start van de scène). Deze tijdvensters kunnen worden geassocieerd met verschillende stadia in het taalproductieproces. Deze resultaten presenteren het eerste neurowetenschappelijk bewijs voor taalproductie-beperkingen in patiënten met klassieke galactosemie in lexicale en syntactische planning - dus voorafgaand aan de output / articulatie-fase (het daadwerkelijk uitspreken van de woorden of zinnen).

In Hoofdstuk 4 hebben we de spatiële aspecten van taalplanning bestudeerd. Functionele neurale netwerken die betrokken zijn bij taalproductie werden onderzocht in een groep gezonde volwassenen. Opnieuw hebben we een soortgelijk paradigma gebruikt, maar ditmaal gecombineerd met functionele magnetic resonance imaging (fMRI) en functionele connectiviteit. Deze technieken kunnen lokaliseren welke hersengebieden actief zijn tijdens een taak 
en welke gebieden functioneel samenwerken. We zagen dat de activiteit in zowel de linker inferieure frontale gyrus (IFG) en het linker pre-supplementaire motorgebied (pre-SMA) steeg met toenemende syntactische complexiteit. Verder hebben we vastgesteld dat deze gebieden elk correleerden met aparte functionele netwerken. De studie toonde daarmee aan dat het taalnetwerk bestaat uit gespecialiseerde functionele sub-netwerken die parallel werken om zich bezig te houden met verschillende aspecten van taalproductie.

In Hoofdstuk 5 hebben we patiënten met klassieke galactosemie en gematchte controles bestudeerd met fMRI tijdens het uitvoeren van dezelfde taalproductietaak. De resultaten toonden aan dat de patiënten een over het algemeen vergelijkbaar netwerk van hersengebieden aanroepen vergeleken met gezonde controles, maar ook aanvullende en meer uitgebreide gebieden. In de patronen van de functionele connectiviteit zagen we een vergelijkbaar resultaat met meer uitgebreide connectiviteit met frontale en motorische gebieden bij de patiënten, en beperktere en zwakkere connectiviteit met superieur temporale gebieden. De gegevens toonden aan dat taalafwijkingen in klassieke galactosemie samenhangen met specifieke veranderingen in het taalnetwerk in de hersenen. Deze veranderingen wijzen op afwijkingen met betrekking tot zowel de syntactische planning en de spraak/motorische planning bij patiënten met klassieke galactosemie.

In Hoofdstuk 6 werd diffusie-gewogen MRI gebruikt om potentiële afwijkingen te onderzoeken in de witte stof microstructuur van patiënten met klassieke galactosemie, vergeleken met gezonde controles. We hebben gebruik gemaakt van een techniek genaamd: neurite orientiation dispersion and density imaging (NODDI). Deze techniek is ontwikkeld om de dichtheid van neurieten / vezelbundels en de spreiding in oriëntatie van deze bundels te onderzoeken (dus in hoeverre ze allen dezelfde kant op lopen of een spreiding of dispersie hebben in de oriëntatie), twee belangrijke onderdelen van de veel gebruikte maat fractionele anisotropie (FA, afgeleid van standaard diffusion tensor imaging - DTI). De resultaten toonden uitgebreide witte stof afwijkingen aan: de neuriet dichtheidsindex (NDI) was lager in de groep patiënten in bilateraal anterieure gebieden, en de oriëntatie dispersie-index (ODI) was hoger in meer posterieure gebieden in de linker hersenhelft. De specifieke regionale profielen zijn over het algemeen in overeenstemming met het cognitieve profiel waargenomen bij klassieke galactosemie, namelijk respectievelijk met hogere orde cognitieve problemen, en taal- en motorische stoornissen. Bovendien waren de witte stof eigenschappen gecorreleerd met ziekte-variabelen (zoals met leeftijd en leeftijd bij aanvang van het dieet) en met gedragsmaten (zoals visueel werkgeheugen). Deze verkennende studie levert de eerste kwantitatieve 
metingen van witte stof afwijkingen, die zowel veranderingen laten zien in de dichtheid en de spreiding in oriëntatie van neurieten. Deze bevindingen bevestigen en breiden voorgaande kwalitatieve observaties van witte stof afwijkingen uit. De gemeten veranderingen in de microstructuur zijn in overeenstemming met abnormale myelinatie, hoewel de verhoogde spreiding in de oriëntatie van neurieten ook deels compensatiemechanismen kan reflecteren.

Tot slot geeft Hoofdstuk 7 een algemene discussie, waar de belangrijkste resultaten en conclusies van de studies uit dit proefschrift worden besproken. Verder worden parallellen getrokken tussen de resultaten en worden vooruitzichten beschouwd. Al met al pionieren we in de toepassing van cognitief neurowetenschappelijk onderzoek op het gebied van klassieke galactosemie. Bovendien laten we de potentie zien van het bundelen van krachten tussen disciplines om tot een beter begrip van deze ziekte te komen. 


$$
m
$$


ACKNOWLEDGEMENTS | DANKWOORD

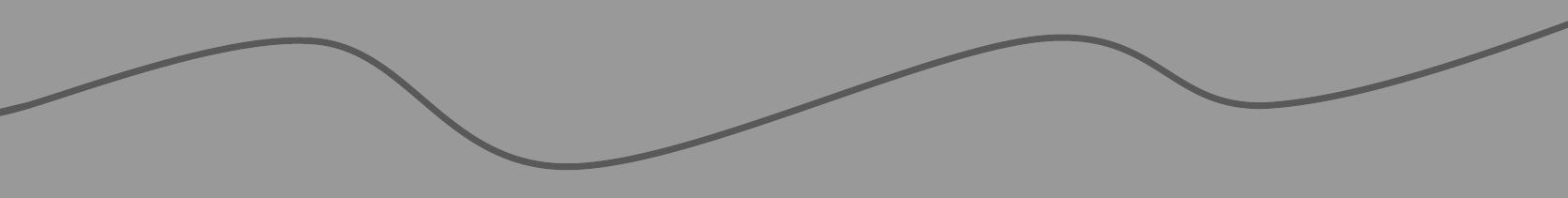



the past few years so this is going to be a truly difficult job.

Who else to start with than the two people that have driven this thesis, motivated me, challenged me, supported me endlessly, and whom I became very fond of: Estela and Bernadette. Estela, jij geloofde al in me vanaf het prille begin. Je gaf me vertrouwen en vele uitdagingen. Zowel op professioneel en persoonlijk vlak heb ik je leren kennen en heb ik ontzettend veel van je geleerd. Bedankt voor de deadlines (ik moet toegeven, ik werk inderdaad goed als er een deadline is, mijn excuses dat het je wellicht af en toe wat gefrustreerd heeft), je vertrouwen, de kansen die je me hebt geboden, onze hardloopavonturen, Indiase avondjes, en alle gezelligheid. Bernadette, like all our email exchanges, this will be a mixture of Dutch and English :). Thank you for your genuine confidence in me, your ability to make me proud of what we have accomplished, the inspiration ("The data looks so beautiful, Inge! Wow! Do you see that?!'), your mentorship, and the feeling that you will have my back. Jullie samen vormden een perfecte mix van 'strengheid', deadlines, doorzetten, oog voor kwaliteit en mogelijkheden, en kansen. Veel dank voor alles! Ook zou ik graag Luc Zimmermann bedanken als tweede promotor. Bedankt voor je oprechte interesse voor mijn werk.

Zonder de deelnemers en de steun van de patiëntenvereniging - Galactosemie Vereniging Nederland - was dit proefschrift niet tot stand gekomen. Veel dank gaat uit naar de patiënten en hun families voor de deelname, het vertrouwen en de genomen moeite. Het was niet altijd even gemakkelijk, sommige zaken hebben mij persoonlijk ook erg geraakt. Ik wens jullie het allerbeste voor de toekomst. Met name Maaike, Jeroen en Ingrid: bedankt voor de logistieke steun en de interesse. Zonder jullie was dit niet gelukt!

Then.. as my entire PhD time has been a mixture of two disciplines, I will split this part up as well. Om te beginnen met de kindergeneeskunde, waar ik chronologisch het eerste binnenliep. Cynthia, mijn voorganger op het gebied van galactosemie, je hebt me ontzettend veel geleerd. Je hebt me de ins and outs laten zien van het onderzoek, de praktische zaken. We hebben samen gereisd en heerlijke cheesecake gedeeld. Ook nadat je was begonnen met een nieuwe baan, hebben we samen lief en leed gedeeld onder het genot van warme chocomel (:) Britt, mijn 'opvolger', ik hoop dat wij deze traditie zullen voortzetten en nog veel gezellige momenten zullen hebben! Dan mijn andere collega's van de kindergeneeskunde, om te beginnen met Sasha, mijn 
kamergenoot en paranimf. Ook al was ik op een gegeven moment niet meer altijd (oftewel: meestal niet) op onze kamer (ons hok) te vinden, ik heb altijd genoten van je gezelschap. Je doorzettingsvermogen werkte inspirerend en ik ben heel trots op wat je bereikt hebt! Ook Ester, Maartje, Kim, Bob, Dillys, Marlou, Marieke, Sylvia en Jesse, bedankt voor de gezellige lunches, verjaardagen en andere collega-momentjes. Ik hoop dat we dit zullen voortzetten, ook nu velen van ons inmiddels een nieuwe weg zijn ingeslagen. Ik hoop dat de woensdagmiddaglunch erin zal blijven! Ook gaat veel dank naar Linda voor het beantwoorden van al mijn vragen en het regelen van vanalles en nog wat! Ook veel dank voor Tamara en Lea voor de ondersteuning, evenals voor Karin en Petra voor de ondersteuning en de interesse. Jörgen, (Jaap) en Jo, van jullie heb ik veel geleerd over de biochemie, genetica en genomics van (diermodellen voor) galactosemie. Ik heb dan ook met veel interesse de vorderingen op het lab gevolgd tijdens de galactosemie-research-meetings. Paul Hofman, bedankt voor de neuro-radiologische ondersteuning in dit project.

Ook gaat mijn dank uit naar de studenten die hebben geholpen met verschillende aspecten van dit proefschrift: Fleur, Kristy en Irene.

Thanks to Estela I was privileged to meet many world experts in the field of galactosemia, be part of the amazingly efficient research symposium at Children's Hospital Boston and many workshops and meetings, and witness the initiation of the European Galactosemia Network. Gerard Berry, Eileen Treacy, Annet Bosch, Nancy Potter, Susan Waisbren, Stephanie Grunewald, Naomi Dale, Ina Knerr, thank you for inspiring me in this field of research.

Then to the other, parallel world of neuroscience. Job, my very first co-author, I very much enjoy your wise advice, our chitchats / mutual complaining, being your brilliant (anonymous) research participant, and generally working with you. Let's keep doing that. Fra, you have taught me so much on programming in Presentation (through the brilliant experimental stimuli you designed for me), on ERP analyses, and on the never-ending ICA analyses. Thank you for investing so much time in helping me with a genuine interest. Many thanks for the other members of the FFA (João, Gojko, still no better name?) group: João, Milene, Gojko, Mario and several very talented students. João, special thanks to you for being such a good travel mate, chauffeur (just kidding $\odot$ ), and pizza baker. I really enjoyed our trips to the language conferences, especially the road-trip in beautiful California which was a conference journey that is going to be hard to top. These trips would also not be the same without our other travel mate and my roommate. Katie, I enjoyed our chats, beach runs / strolls, and your vision on science and careers in general. I hope we stay in touch! 
Dankzij Anke Sambeth heb ik - als student-assistent - mijn eerste echte ervaringen in de onderzoekswereld mogen meemaken. lk heb met veel plezier gewerkt aan de EEG metingen, de EEG analyses (inclusief het uitvogelen van batch-processing samen met Moos) en de rest wat erbij komt kijken. Veel dank daarvoor! Mede hierdoor blijf ik ook interesse houden in de neurofarmacologie, wie weet doe ik er ooit nog wat mee!

Thank you Anke en Thomas (and Mario for a while) for sharing your office with me. For you it is the other way around: at first I was hardly ever there until I started to spend my time on the MRI analyses. Anke, I learned a lot from you regarding small analysis tips and tricks, and I enjoyed our chats. Thomas, you patiently endure all my ICT/VM-questions, thank you for that. I always enjoy hearing your stories, what you are working on, and so forth. I am glad we can keep doing that!

Also many thanks to my other $\mathrm{CN}$ colleagues for the fun, the chats, the Friday afternoon themed borrels (although short-lived), the parties and the great atmosphere: Anna G, Sanae, Judith, Joost, Sanne, Arne, Mario, Anna Z, Lars, Matteo, Valentin, Nina, Christianne, Franzi, Tamara, Emma, thank you! Special thanks to Christl and Riny for your help, to Kamil and Fabrizio for the support on the ASL data and analyses, to Amanda for exchanging experiences on our projects (and to you and Jeroen for giving me the confidence to start a new challenge), Lotty and Chris Wiggins for support with scanning, to Alard and Matteo for working together on the DWI data. Also, Gary, thank you for welcoming me in London and for teaching me everything on DW analyses, command line processing, and on the amazing food London has to offer. I very much enjoyed the time I spent in your group. Also, Uran, Andrada, Maira, Lebina, Francesco, Tingting - you really made my stay a lot more fun, many thanks to you! Let's stay in touch! Hester, John and Sophie, Karin, Yacob and Jojo, and Bea - your company was invaluable as well!

Sanne, mijn paranimf, vriendin en collega, we hebben elkaar tijdens onze allereerste onderwijsgroep ontmoet en hebben sindsdien het beste in elkaar naar boven gehaald. Je hebt me altijd gemotiveerd en met jou kon ik alles bespreken. Ik vind het fijn dat we veel raakvlakken hebben in ons werk, waardoor we dit na de studie door konden zetten. Ik ben heel blij dat je achter me zult staan als paranimf en hoop dat we nog lang vriendinnen zullen blijven! Conny, mijn werkbuddy, met jou kan ik ook eeuwig blijven praten over onze analyses. Ik bewonder wat jij allemaal voor elkaar krijgt met je harde werken! Ik vind het heerlijk om met jou te kletsen over vanalles en nog wat onder het genot 
van een lekkere kop koffie. Laten we dat blijven doen! Ook Renate, Bea, fijn dat het met jullie nog altijd zo gezellig is!

Samen met mijn collega's van de SOAP Foundation proberen we ook de minder bedeelden een kans te geven op onderwijs en een beter leven. Elham, Ehsan, Elaha, Saboor en de rest - al zijn we een stuk minder actief tegenwoordig, ik haal er nog steeds voldoening uit.

Zonder mijn vriendinnen-club (sorry voor de benaming Daine :p, maar jij hoort hier ook bij) zou mijn leven een stuk saaier zijn. Kirs, ik ken je al bijna mijn hele leven. Je bent er altijd voor me en ik ben trots op waar jij nu staat! Je kunt het, hoe zwaar het ook af en toe is! Soeï, jij bent ook hard op weg om te komen waar je wilt zijn. Jouw nuchterheid en humor zijn uniek. Ik hoop dat we nog vele gezellige momenten zullen hebben, samen met Amy en de guys. Vivian, bedankt voor jouw interesse en de vele gezellig avondjes. Rachel, voor de gezelligheid en de leuke wandelingen met de hondjes. Ook Maxine, mijn grootste fan $(-)$, bedankt voor je interesse en gezelschap (al mogen we elkaar wel wat vaker gaan zien!). Kevin, Maria en de kids, bedankt voor jullie vriendschap!

Ook gaat grote dank uit naar mijn (schoon)familie. Zonder jullie was ik nooit gekomen waar ik nu ben. Bedankt Kirs, Jimmy en lieve Senna en Josi voor het plezier dat jullie me geven! Pap en mam, jullie zijn er altijd voor me. Bedankt voor alles.

Het laatste en meest speciale plekje is voor jou, Kevin. Je bent er al altijd voor me, met trots en steun. Ik maak het je niet altijd even gemakkelijk, maar ik ben blij dat je achter me staat en me de ruimte geeft als mijn ideeën en plannen die vereisen. Met je zorgzame, nuchtere en realistische blik op het leven, werk en de wetenschap, vorm je een perfecte tegenhanger en herinner je me aan wat écht belangrijk is. Jij en Lara geven me een thuis, daarvoor mijn eeuwige dank en liefde! $\bullet$ 

Inge Timmers was born on July $3^{\text {rd }} 1987$ in Sittard, the Netherlands. She graduated from high school (gymnasium) at Trevianum in Sittard in 2005, after which she started the Bachelor programme in Psychology at Maastricht University. She was selected for the Honours program, which she successfully completed. She graduated Cum Laude in 2008 and was accepted for the Research Master in Cognitive and Clinical Neuroscience (Neuropsychology track). In the

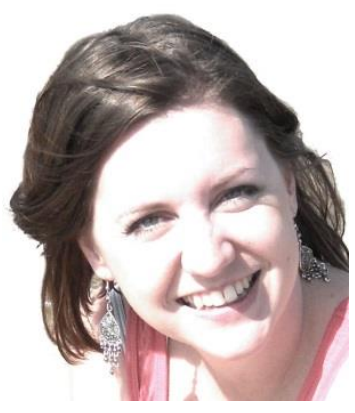
meantime, she worked as a student-assistant with Dr. Anke Sambeth, where she was trained in research and electroencephalography in combination with neuro-pharmacological manipulations. She received a top $3 \%$ award with respect to the first year of the Research Master and graduated Cum Laude in 2010 after finishing a research internship with Prof. Dr. Bernadette Jansma and Dr. Estela Rubio-Gozalbo on classic galactosemia, and a clinical neuropsychological internship and minor at MeanderGroep Zuid-Limburg. In 2011, she started to work as a bi-faculty PhD candidate under the supervision of Dr. Estela Rubio-Gozalbo at the Department of Paediatrics, Maastricht University Medical Center, and Prof. Dr. Bernadette Jansma at the Department of Cognitive Neuroscience, Maastricht University. In 2013, she visited the Microstructure Imaging Group at University College London, UK, for three months to collaborate on the analysis of diffusion weighted data. Currently, she started to work as a post-doctoral researcher on neurophysiological effects of rehabilitation of chronic pain patients with Dr. Jeroen de Jong and Dr. Amanda Kaas at the Department of Rehabilitation Medicine, and Department of Cognitive Neuroscience, Maastricht University. 

Timmers, I., van den Hurk, J., Di Salle, F., Rubio-Gozalbo, M. E. and Jansma, B. M. (2011). Language production and working memory in classic galactosemia from a cognitive neuroscience perspective: future research directions. Journal of Inherited Metabolic Disease, 34(2), 367-376.

Waisbren, S. E., Potter, N. L., Gordon, C. M., Green, R. C., Greenstein, P., Gubbels, C. S., Rubio-Gozalbo, E., Schomer, D., Welt, C., Anastasoaie, V., D'Anna, K., Gentile, J., Guo, C., Hecht, L., Jackson, R., Jansma, B. M., Li, Y., Lip, V., Miller, D. T., Murray, M., Power, L., Quinn, N., Rohr, F., Shen, Y., Skinder-Meredith, A., Timmers, I., Tunick, R., Wessel, A., Wu, B., Levy, H., Elsas, L., and Berry, G. T (2012). The adult galactosemic phenotype. Journal of Inherited Metabolic Disease, 35(2), 279-286.

Timmers, I., Jansma, B. M. and Rubio-Gozalbo, M.E. (2012). From mind to mouth: event-related potentials of sentence production in classic galactosemia, PLOS ONE, 7(12), e52826.

Timmers, I., Gentile, F., Rubio-Gozalbo, M. E. and Jansma, B. M. (2013). Syntactic encoding during overt sentence production: an event-related potential study, PLOS ONE 8(12): e82884.

Timmers, I., Zhang, H., Bastiani, M., Jansma, B.M., Roebroeck, A., and RubioGozalbo, M.E. (submitted). Assessing white matter microstructure in classic galactosemia using neurite orientation dispersion and density imaging

Timmers, I., van den Hurk, J., Hofman, P.A.M., Zimmermann, L.J.I., Uludağ, K., Jansma, B.M., and Rubio-Gozalbo, M.E. (submitted). Neural networks related to syntactic and motor speech planning are affected in classic galactosemia.

Timmers, I., van den Hurk, J., Rubio-Gozalbo, M.E., and Jansma, B.M. (in preparation). The functional neural network of sentence production delineates into syntactic and motor speech planning networks: an fMRI connectivity study

Timmers, I., Roebroeck, A., Bastiani, M., Jansma, B.M., Rubio-Gozalbo, M.E., and Zhang, $\mathrm{H}$ (in preparation). Assessing microstructural substrates of white matter abnormalities using NODDI - application to a metabolic disease 
